Tel-Aviv University

Raymond and Beverly Sackler

Faculty of Exact Sciences

\title{
High luminosity operation of large solid angle scintillator arrays in Jefferson Lab Hall A
}

\author{
Thesis submitted toward the M.Sc. degree \\ Department of Physics and Astronomy \\ Tel-Aviv University \\ by \\ Ran Shneor
}

This research work was carried out under the supervision of Professor Eli Piasetzky

December 2003 


\section{Acknowledgments}

I wish to express my deep thanks to my advisor, Prof. Eli Piasetzky, for his constant advice and support in all respects, and for his patience. Many thanks to Dr. D. Higinbotham, Peter Monaghan, Dr. S. Wood, Dr. O. Gayou, and to all the people which helped make my work at TJNAF possible. 


\begin{abstract}
This thesis describes selected aspects of high luminosity operation of large solid angle scintillator arrays in Hall A of the CEBAF (Central Electron Beam Accelerator Facility) at TJNAF (Thomas Jefferson National Accelerator Facility ). CEBAF is a high current, high duty factor electron accelerator with a maximum beam energy of about $6 \mathrm{GeV}$ and a maximum current of $200 \mu \mathrm{A}$. Operating large solid angle scintillator arrays in high luminosity environment presents several problems such as high singles rates, low signal to noise ratios and shielding requirements. To demonstrate the need for large solid angle and momentum acceptance detectors as a third arm in Hall A, we will give a brief overview of the physics motivating five approved experiments, which utilize scintillator arrays.

We will then focus on the design and assembly of these scintillator arrays, with special focus on the two new detector packages built for the Short Range Correlation experiment E01-015. This thesis also contains the description and results of different tests and calibrations which where conducted for these arrays. We also present the description of a number of tests which were done in order to estimate the singles rates, data reconstruction, filtering techniques and shielding required for these counters.
\end{abstract}




\section{Contents}

1 Introduction $\quad 7$

1.1 Scientific Motivation ... . . . . . . . . . . . . 7

1.1.1 E01-015: Studying the Internal Small-Distance Structure of Nuclei via the Triple Coincident (e,e'pN) Measurement. 9

1.1.2 E02-013: Measurement of the Neutron Electric Form Factor $G_{n}^{E}$ at High $Q^{2}$. . . . . . . . . . . . . . 10

1.1.3 E02-108: Measurement of $\mathrm{A}_{x}$ and $\mathrm{A}_{z}$ asymmetries in the quasi-elastic polarized ${ }^{3} \vec{H} e\left(\vec{e}, e^{\prime} \mathrm{d}\right)$ reaction. . . . . . . . . 13

1.1.4 E02-101: Exclusive Study of Deuteron Electro-disintegration Near Threshold. . . . . . . . . . . . . . . . . . 14

1.1.5 E01-014: Precision Measurement of Electro-production of $\pi^{0}$ near Threshold: A Test of Chiral QCD Dynamics. . . 14

1.1.6 Summary .......................... 16

1.2 Scintillators Operation . . . . . . . . . . . . . . 16

1.2.1 Photomultiplier Tube .............. 17

1.2.2 Momentum and position measurement . . . . . . . . . . . 20

1.2.3 Expected resolution ............... . 21

\section{Scintillator planes for the BigBite} spectrometer 22

2.1 BigBite spectrometer . . . . . . . . . . . . . . . . . 22

2.1.1 Design and building of scintillator planes for BigBite . . . 22

2.2 The Auxiliary plane . . . . . . . . . . . . . . . . 22

2.2.1 The PhotoMultiplier Tubes . . . . . . . . . . . . 23

2.2 .2 Scintillators . . . . . . . . . . . . . . . 23

2.2.3 Light guides . . . . . . . . . . . . . . . . . . . . 24

2.2.4 Magnetic shielding ................ . 24

2.3 The Trigger plane . . . . . . . . . . . . . . . . 26

2.3.1 The Photomultiplier Tubes . . . . . . . . . . . . 26

2.3.2 Scintillators .................. 26

2.3.3 Light guides . . . . . . . . . . . . . . . . 26

2.4 Tests and Performance . . . . . . . . . . . . . . . 29

2.5 Auxiliary plane testing . . . . . . . . . . . . . . . . 29

2.5.1 Analog-Digital-Converter (ADC) spectrum analysis . . . . 29

2.5.2 TDC spectrum analysis . . . . . . . . . . . . . 30

2.6 Trigger plane testing . . . . . . . . . . . . . . . . . 34

2.7 Complete detector package testing . . . . . . . . . . . . . . 38

2.7.1 Angular distribution of cosmic rays . . . . . . . . . . . . 39

2.7.2 Measurement of the hit position across the Trigger Plane 39

2.8 Simulations of BigBite Performances . . . . . . . . . . . . . . . . 40

2.8.1 Simulated momentum and scattering angle reconstruction with BigBite . . . . . . . . . . . . . . . . . 41

2.8.2 Momentum reconstruction using TOF . . . . . . . . . 41 
2.8.3 Momentum and scattering angle reconstruction using the Hit Position . . . . . . . . . . . . . . . 45

2.8.4 Position and TOF combined algorithm . . . . . . . . . . . 46

2.8.5 Particle Identification . . . . . . . . . . . . . . . . . . 46

2.8.6 Singles rates tests and simulations . . . . . . . . . 51

3 Neutron array for the SRC experiment 53

3.1 Design and building of the neutron-array for the SRC experiment 53

3.1.1 The neutron scintillator planes . . . . . . . . . . 53

3.1 .2 The Veto layer. . . . . . . . . . . . . . . . . . . 55

3.2 Tests of Performance . . . . . . . . . . . . . . . . . 55

3.2.1 High Voltage setting ............. 55

3.2.2 Position calibration. . . . . . . . . . . . 57

3.2.3 TOF calibration ................ 61

3.2.4 Attenuation length studies . . . . . . . . . . . . 62

3.2.5 Summary and conclusions . . . . . . . . . . . . 62

3.3 The neutron array singles rates and shielding test . . . . . . . . . 62

\section{List of Figures}

1 The Thomas Jefferson National Accelerator Facility (TJNAF). . 8

2 The High Resolution Spectrometer at Hall A. . . . . . . . . . . . 8

3 Impulse approximation description of the short range correlation

experiment. . . . . . . . . . . . . . . . . 9

Kinematic of the short range correlation experiment. . . . . . . . 10

Experimental setup of the short range correlation experiment. . . 11

MCEEP simulated results of the SRC experiment. . . . . . . . . 12

The $G_{n}^{E}$ Experimental set-up. . . . . . . . . . . . . . . 13

E02-108 Experimental set-up. . . . . . . . . . . . . 14

E02-101 Experimental kinematics. . . . . . . . . . . . . 15

10 E01-014 Experimental set-up. . . . . . . . . . . . . . 15

11 A schematic description of a BigBite scintillator. . . . . . . . . 18

12 Emission and Quantum efficiency spectra . . . . . . . . . . . 18

13 A schematic description of a PMT. . . . . . . . . . . . . . . 19

14 A schematic side view of BigBite, a few proton tracks are also shown. . . . . . . . . . . . . . . 23

15 A CAD plot of the auxiliary plane. . . . . . . . . . . . 24

16 Schematic views of the an auxiliary plane scintillator. . . . . . 25

17 Schematic views of the an auxiliary light-guide, PMT and a soft iron magnetic shielding cylinder. . . . . . . . . . . . . . . 25

18 A CAD plot of the trigger $\mathrm{E}$ and $\Delta \mathrm{E}$ planes. . . . . . . . . . . . 27

19 Schematic diagram of the $\mathrm{E}$ and $\Delta \mathrm{E}$ trigger plane scintillator. . . 28

20 Schematic diagram of the $\mathrm{E}$ (left) and $\Delta \mathrm{E}$ (right) light-guides. $\quad 28$

21 Schematic diagram of the $\mathrm{E}$ and $\Delta \mathrm{E}$ trigger plane scintillators mounting. . . . . . . . . . . . . . . . . 29 
22 Schematic diagrams of the assembly of the trigger plane. . . . . . 30

23 The setup used for testing the auxiliary plane with cosmic rays. . 31

24 ADC (left) and TDC (right) spectrum for a typical auxiliary de-

tector. . . . . . . . . . . . . . . . . . 32

25 Cosmic ray events as viewed using an Oscilloscope. . . . . . . . . 32

26 The difference between the pedestal and the cosmic peak positions for all the counters. . . . . . . . . . . . . . . . 33

27 ADC correction. . . . . . . . . . . . . . . . 33

28 TDC peak . . . . . . . . . . . . . . . . . . . 34

29 The different setups used for the trigger plane testing. . . . . . . 35

30 The calculated time resolution for the E counters. . . . . . . . 36

31 The calculated time resolution for the $\Delta \mathrm{E}$ counters. . . . . . . . 36

32 Typical TOF spectrum between an Auxiliary plane counter and a $\Delta \mathrm{E}$ counter before corrections. . . . . . . . . . . . . . . . . . . 37

33 A typical TOF/Distance spectrum after all corrections (see text). 38

34 TOF/Meter [ns/meter] between the Auxiliary and the Trigger plane. . . . . . . . . . . . . . . . . . . 39

35 Cosmic rays angular distribution . . . . . . . . . . . . . . . . 40

36 Measured position along counters . . . . . . . . . . . . . . 41

37 Reconstructed Momentum for protons with momentum of 300, 400 and $500 \mathrm{MeV} /$ c. . . . . . . . . . . . . . . . . . 44

38 A Trajectory of a proton in BigBite. . . . . . . . . . . . . 46

39 Momentum reconstruction using the analytical formula, based on hit positions. . . . . . . . . . . . . . . . . . . 47

40 Neural-Network reconstruction, based on hit positions. . . . . . . 48

41 TOF reconstructed momentum before (left) and after (right) position TOF consistency cut. . . . . . . . . . . . . . . . 49

42 Particle Identification. . . . . . . . . . . . . . . . . . . . 50

43 Measured (points) and calculated (dashed line) hit rates in $\mathrm{Hz}$ versus the proton momentum in $\mathrm{MeV} /$ c. . . . . . . . . . . . . . . 51

44 A picture of the neutron array, in the test area at TJNAF. . . . 53

45 A schematic side view of the neutron array. . . . . . . . . . . 54

46 A schematic side view of the different scintillators bars used for the neutron array. . . . . . . . . . . . . . . . 56

47 A schematic view of the veto layer in-front of the neutron array. 57

48 Top view of the neutron array . . . . . . . . . . . . . . 58

49 Different experimental setups for the cosmic rays tests. . . . . . . 59

50 Typical ADC spectrum for cosmic ray going across the counter as shown in figure 6 A. . . . . . . . . . . . . . . . . 60

51 Measured position along the entire front plan. . . . . . . . . . 61

52 Timing resolution $(\sigma)$ for each counter in the array. . . . . . . 63

53 Top: TOF $/ \mathrm{m}$ for a typical P1 counter - before the shift and calibrations. Bottom - a single counter peak after shift. . . . . . 64

54 ADC (right) versus position along the counters. . . . . . . . . 65

55 The different shielding configurations used for the shielding test. 66 
56 The ratio of the number of counts from bar \#3 (room) to bar \#1

(target) as a function of threshold energy of the incoming neutron. 67

\section{List of Tables}

1 The momentum reconstruction resolution, using simulated TOF between auxiliary plane and the trigger plane, and between the target and the trigger plane. . . . . . . . . . . 70

2 Test versus Simulation parameters. . . . . . . . . . . . . 70

3 Measured and calculated rates. . . . . . . . . . . . . 70

4 The number of counts from each bar with cosmic rays. . . . . . . 71

5 The parameters and results from the shielding test. . . . . . . . 71 


\section{Introduction}

\subsection{Scientific Motivation}

The Central Electron Beam Accelerator Facility (CEBAF) is a super-conducting high current, high duty factor electron accelerator with a maximum beam energy of about $6 \mathrm{GeV}$ (see figure 1). The polarized source provides up to $78 \%$ polarized electrons with a maximum current of $200 \mu \mathrm{A}$ which are preaccelerated to $45 \mathrm{MeV}$ by the injector. The beam is then further accelerated by the two main linacs, which are connected by $180^{\circ}$ recirculation arcs. Up to five re-circulations (5-pass) are possible. Each linac consists of three cryo modules, each containing eight cryo units consisting of five cell elliptical cavities. The cryo-units are made of niobium and are cooled to $2^{\circ} \mathrm{K}$. They are driven by an RF frequency of $1.497 \mathrm{GHz}$. Three beams can be accelerated simultaneously and are divided in the beam switch-yard to three experimental halls, A, B and $\mathrm{C}$ [1].

Currently Hall A has two High Resolution Spectrometers (HRS) (See figure 2). Each HRS detector stack is equipped with two Vertical Drift Chambers (VDC), each containing two wire planes. The first VDC is placed close to the optical focus of the HRS, while the second one is offset $50 \mathrm{~cm}$ downstream. This ensures good resolution for the determination of the tracking angle $(6 \mathrm{mr}$ horizontally and $2 \mathrm{mr}$ vertically). The momentum resolution of each of the HRS is $10^{-4}$ (FWHM). The angular acceptance of each of the HRS is approximately $6 \mathrm{msr}$. The Momentum acceptance $(\Delta \mathrm{p} / \mathrm{p})$ of each spectrometer is $\pm 4.5 \%$.

These parameters result in a target position resolution on the order of a few $\mathrm{mm}$.

In each HRS, there are three scintillator planes (S1, S2 and S3) which are responsible for triggering the Data Acquisition System (DAQ). Particle Identification is achieved by Gas Cerenkov Counters, Aerogel Cerenkov Counters, and Lead Glass Total Absorption Shower Detectors. The spectrometers are used with different triggering modes, to enable both single and double arm measurements. Typical measurements with these two high resolution, small acceptance spec-

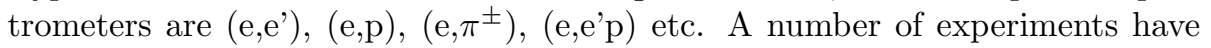
been proposed, which will require the use of a third arm either for applying triple coincidence between the scattered electron and an additional two particles, or for doing measurements which require much larger momentum or angular acceptances than provided by the HRS's.

A large acceptance spectrometer aptly named "BigBite" originally used at NIKHEF at the Netherlands, is now being prepared for use in Hall A. Having a Momentum acceptance of 200-900 MeV/c and a solid angle of $96 \mathrm{msr}$, BigBite is an ideal candidate to complement the two HRS, in measuring charged particles. Scintillator arrays were also designed and built for large angle detection of neutral particles and specifically for the detection of neutrons. More details on Hall $\mathrm{A}$ and its equipment can be found in ref [1]. 


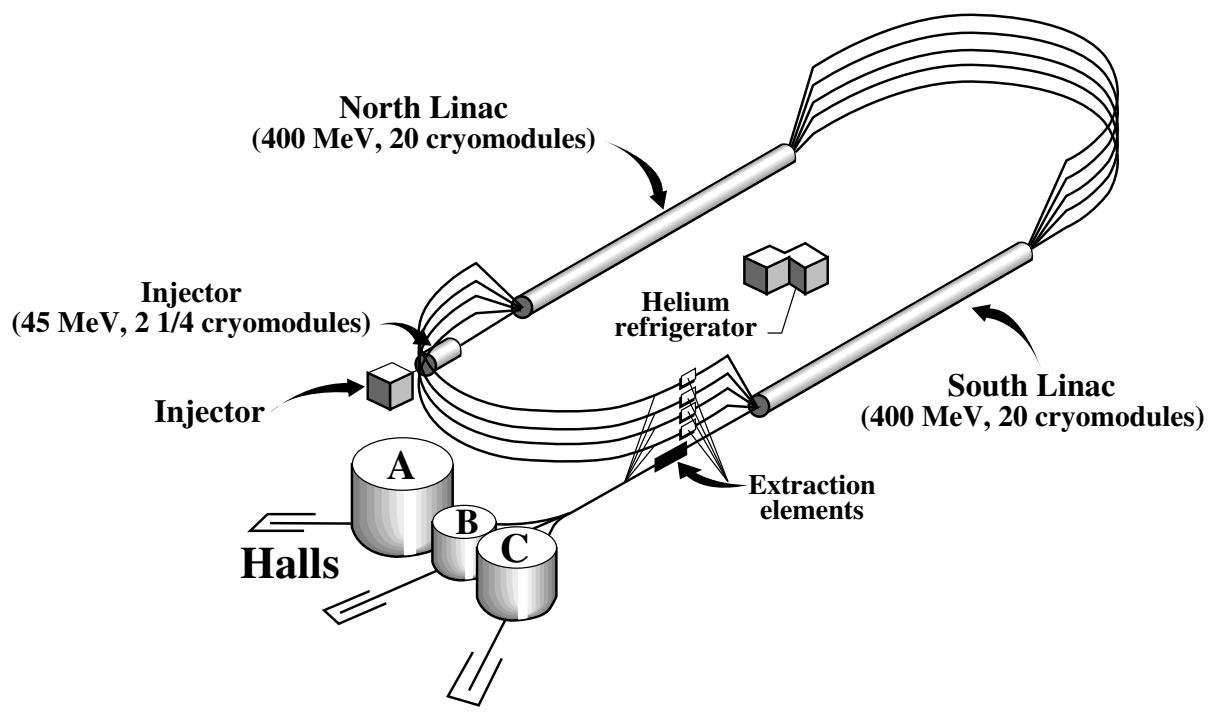

Figure 1: The Thomas Jefferson National Accelerator Facility (TJNAF). The three experimental Halls A, B, and C can be seen on the bottom left.

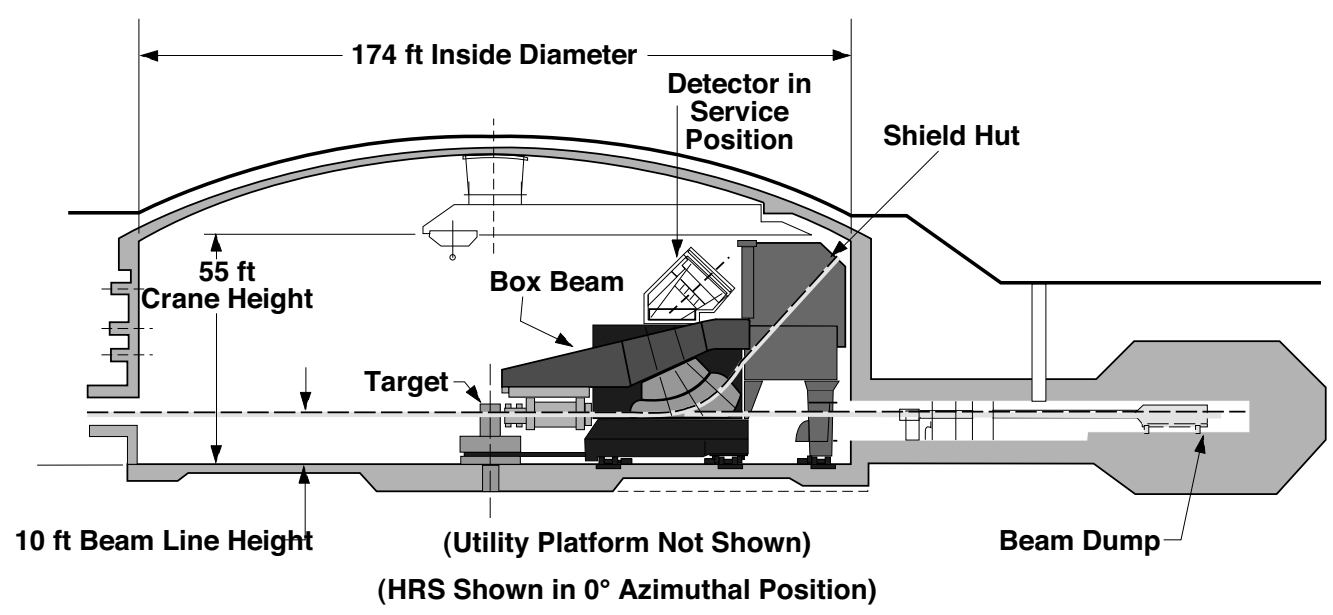

Figure 2: The High Resolution Spectrometer at Hall A. 


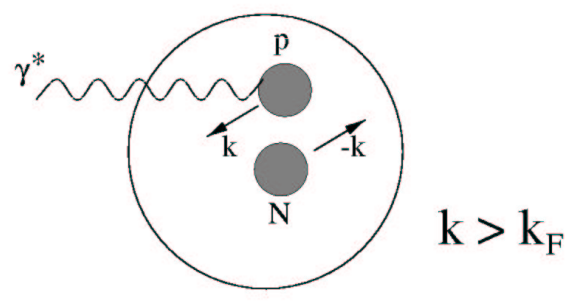

Figure 3: Impulse approximation description of the short range correlation experiment. The large momentum, $\mathrm{k}>k_{F}$ is due to the spacial proximity of the two nucleons in the nucleus. $\mathrm{N}$ refers to either a proton or a neutron.

To demonstrate the potential use of a large solid angle and momentum acceptance third arm in Hall A, we give a brief overview of the physics motivating five experiments already approved. These experiments will utilize the BigBite spectrometer and/or scintillator arrays as large solid angle detectors, to investigate a broad range of nuclear physics.

\subsubsection{E01-015: Studying the Internal Small-Distance Structure of Nuclei via the Triple Coincident (e,e'pN) Measurement.}

A nucleon-nucleon short range correlation occurs when the nucleon wave functions strongly overlap inside the nucleus [2]. In the impulse approximation description of Short Range Correlation (SRC), a virtual photon can be absorbed by one nucleon and the correlated partner (if there is one) is emitted, with high momenta, in the opposite direction, as illustrated in figure 3 .

Using a ${ }^{12} \mathrm{C}$ target, the scattered electrons will be detected by one HRS, the struck proton will be measured by the other HRS, and the correlated partner (if there is one) will be measured either by BigBite (for a proton ), or using a neutron array (for a neutron). This will allow us to measure the fraction of (e,e'p) events in which correlated nucleons (i.e pp or pn) are observed, as a function of the initial momentum of the proton in the nucleus. Two large acceptance detector packages are needed, for the measurement of both protons and neutrons in coincidence with the scattered electron and struck proton.

The short range correlation experiment will provide data at $\mathrm{Q}^{2}=2 \mathrm{GeV} / \mathrm{c}^{2}$ over a range of recoil proton momenta of $250-600 \mathrm{MeV} / \mathrm{c}$ and will explore the region of $x_{B}>1$ where there is currently very little data. The detailed kinematics we choose are shown in Figure 4. The experimental setup with the two HRSs, BigBite and neutron-array is shown schematically in Figure 5.

The BigBite detector package for this experiment consists of 3 scintillator planes (Auxiliary, E plane and the $\Delta \mathrm{E}$ plane ). The Auxiliary plane consists of 56 scintillators bars $\left(350 \times 25 \times 2.5 \mathrm{~mm}^{3}\right)$ with each bar coupled to a PMT on one end [3]. The E plane consists of 24 bars $\left(500 \times 86 \times 30 \mathrm{~mm}^{3}\right)$, with a PMT coupled to both ends of each bar. The $\Delta \mathrm{E}$ plane also consists of 24 bars $(500 \times 86 \times 3$ 


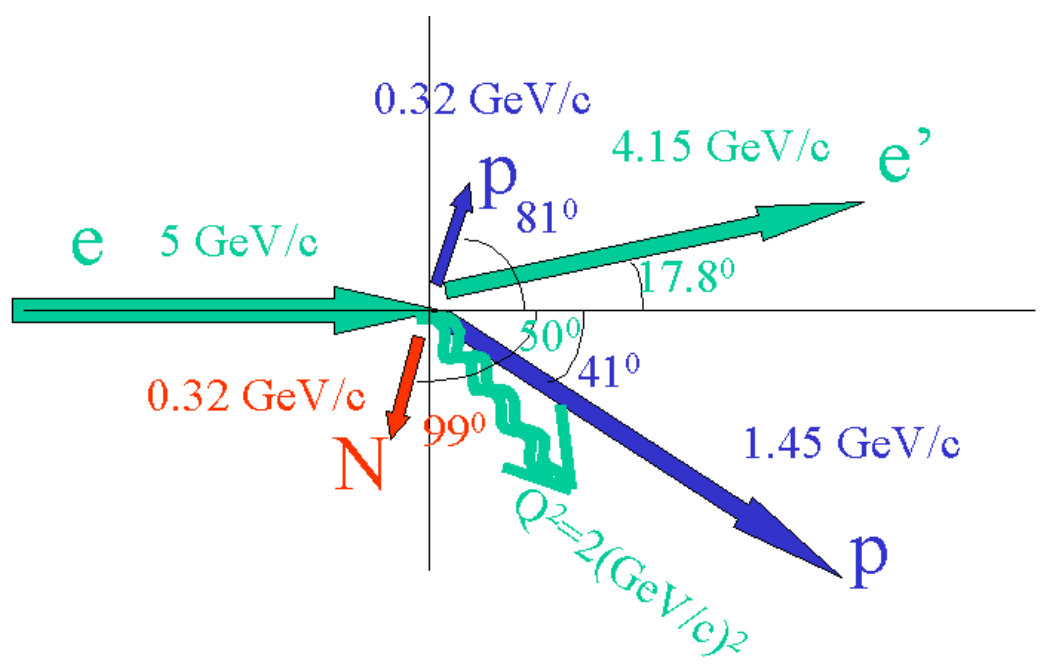

Figure 4: Kinematic of the short range correlation experiment.

$\mathrm{mm}^{3}$ ), with a PMT coupled to both ends of each bar [4]. The detailed design and performance of these large solid angle scintillator arrays in BigBite will be presented in chapter 2 of this thesis.

In order to understand the angular and momentum acceptance needed to measure the correlated nucleon, a monte-carlo simulation (MCEEP) was used [5]. In figure 6 we show the expected momentum and angular distribution of the correlated nucleon, using the kinematics shown in figure 4.

The correlated partner momentum and angular distributions shown in figure 6 , determine the acceptance of the detector package needed. With these kinematics, the detector needed to measure the full distribution of the correlated partner nucleon should have a momentum acceptance in the range $150-400$ $\mathrm{MeV} / \mathrm{c}$, and an Out-of-plane angular acceptance of about $\pm 40^{\circ}$.

\subsubsection{E02-013: Measurement of the Neutron Electric Form Factor $G_{n}^{E}$ at High $Q^{2}$.}

The neutron electric form factor, $G_{n}^{E}$ is related to the charge distribution of quarks in the neutron. Very little is known about $G_{n}^{E}$ at high $\mathrm{Q}^{2}$. As free neutron targets do not exist, the values of $G_{n}^{E}$ will be extracted from the measured asymmetry in the cross-section of the doubly polarized ,quasi-elastic ${ }^{3} \mathrm{He}(\mathrm{e}, \mathrm{e}$ 'n) reaction (see figure 7 ).

The scattered electrons with an energy range of $1200-1500 \mathrm{MeV} / \mathrm{c}$ will be detected in the BigBite spectrometer. A new large solid angle scintillator array, matched to the BigBite acceptance, will be used to detect the high momentum 


\section{Experimental setup}

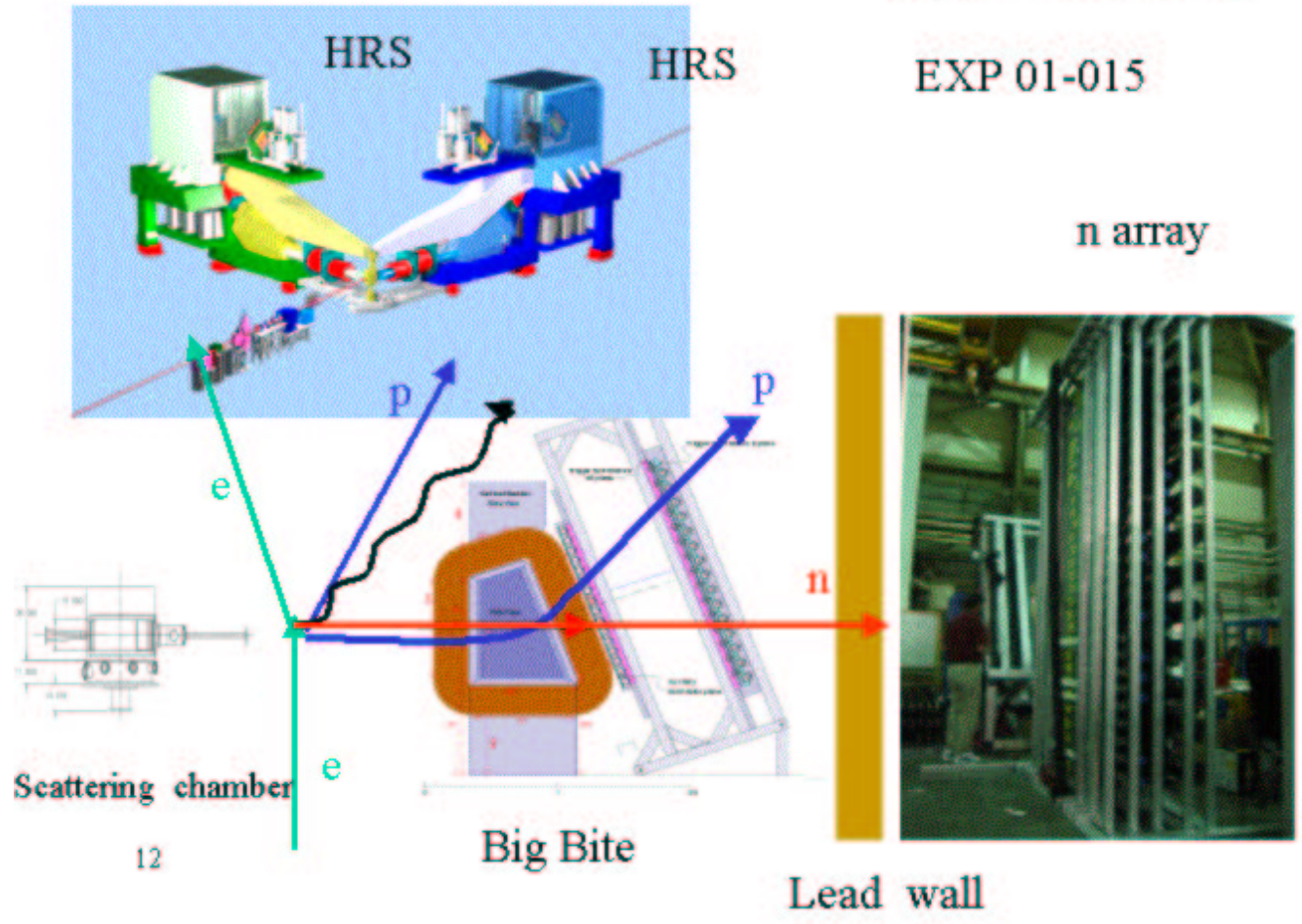

Figure 5: Experimental setup of the short range correlation experiment. 

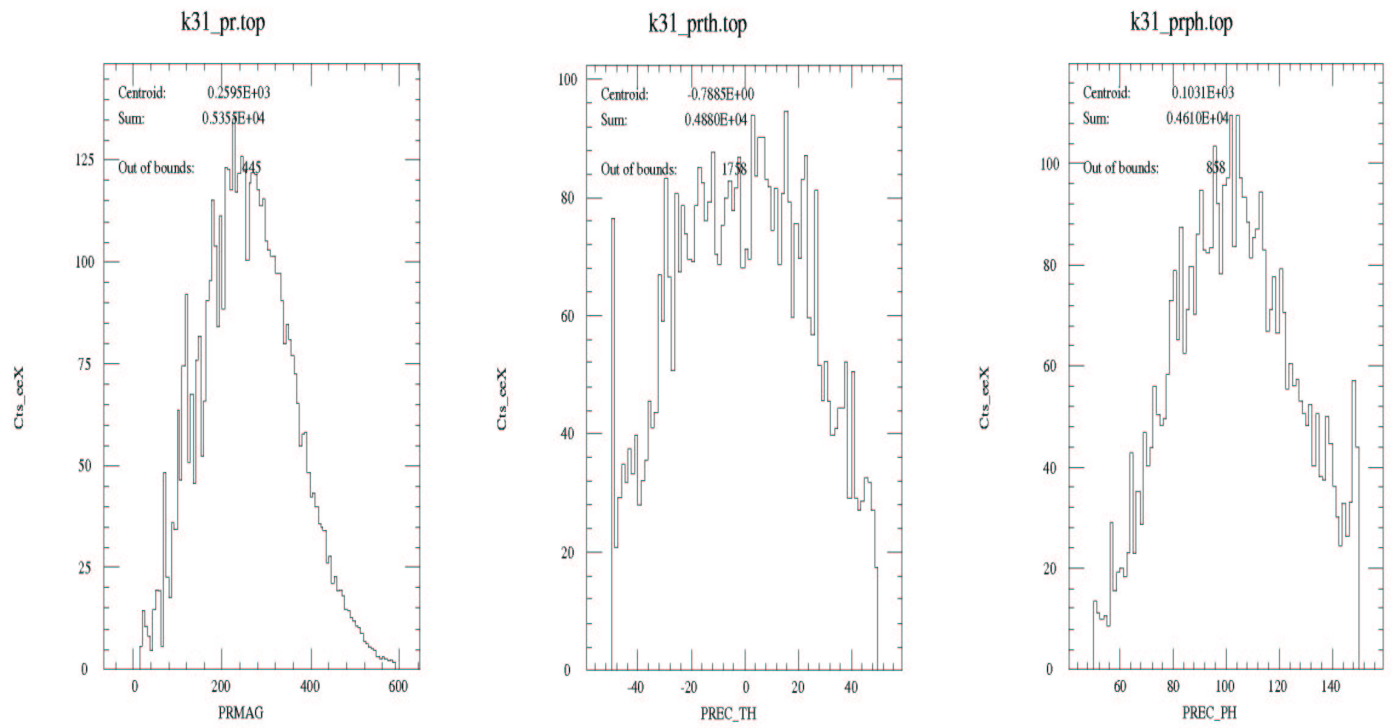

Figure 6: MCEEP simulated results of the SRC experiment.

Left - Momentum distribution of the correlated scattered nucleon (shown as $\mathrm{N}$ in fig. 4) in $[\mathrm{MeV} / \mathrm{c}]$. Center - Out-of-plane angular distribution of the correlated scattered nucleon [deg]. Right - In-plane angular distribution of the correlated scattered nucleon [deg]. 


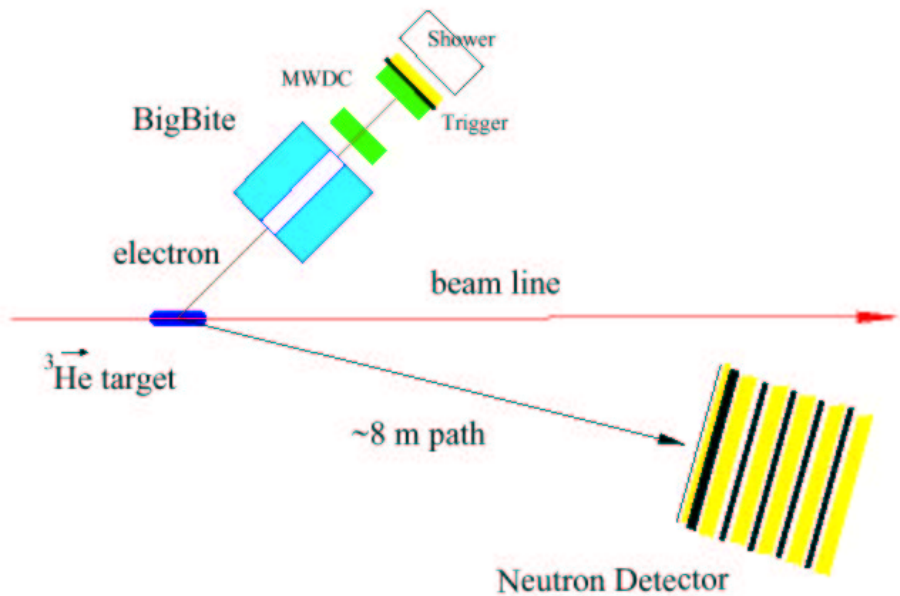

Figure 7: The $G_{n}^{E}$ Experimental set-up.

$(>1 \mathrm{GeV} / \mathrm{c}$ ) recoiling neutrons. The detection of the large momentum neutrons over high level of background radiation (singles rates), in this experiment, is achieved by setting sufficiently high detection thresholds. The neutron detector will have layers of lead positioned between the scintillator planes. This maximizes the energy deposited in the detector, and enables setting sufficiently high detection thresholds. The relation between singles rates and detection thresholds is discussed later in this work (see section 3.3). The typical detection threshold considered in the SRC experiment is $5-15 \mathrm{MeV}$. For this experiment, the total energy deposit threshold is about $150 \mathrm{MeV}[6]$.

\subsubsection{E02-108: Measurement of $\mathbf{A}_{x}$ and $\mathbf{A}_{z}$ asymmetries in the quasi- elastic polarized ${ }^{3} \overrightarrow{H e}\left(\vec{e}, e^{\prime} \mathbf{d}\right)$ reaction.}

The ground state of ${ }^{3} \mathrm{He}$ is predicted to consist of S, S' and D state components. By measuring the cross-section asymmetries $\mathrm{Ax}$ and $\mathrm{Az}$, in both parallel and perpendicular kinematics, the size of the S' and D states can be determined [7]. This experiment will test the Faddeev calculations of the three-body system and increase our knowledge of the role of the S' and D states in ${ }^{3} \mathrm{He}$. The out-of-plane acceptance of BigBite, is utilized to measure the asymmetry Ae (out of plane asymmetry) simultaneously, during the Ax and Az measurements. BigBite will allow for simultaneous measurements of ${ }^{3} \mathrm{He}\left(\mathrm{e}, \mathrm{e}\right.$ 'p)d and ${ }^{3} \mathrm{He}(\mathrm{e}, \mathrm{e}$ 'p $)$ pn channels, while each of the two HRSs will be used the measure the scattered electron for each of these channels (see figure 8 for the planned setup). 


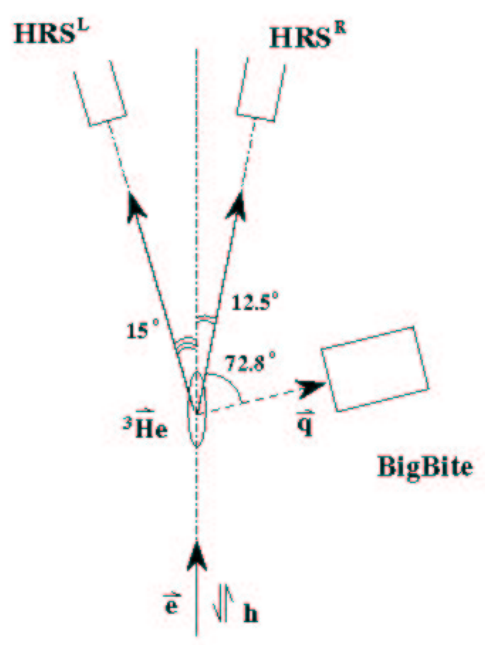

Figure 8: E02-108 Experimental set-up.

\subsubsection{E02-101: Exclusive Study of Deuteron Electro-disintegration Near Threshold.}

This experiment [8] utilizes the acceptance of BigBite to make out-of-plane measurements of the structure functions $\mathrm{R}_{l}, \mathrm{R}_{t}, \mathrm{R}_{t t}, \mathrm{R}_{l t}$ in the reaction $\mathrm{d}(\mathrm{e}, \mathrm{e}$ 'p)n. In the proposed kinematics range of $\mathrm{Q}^{2}=12 \mathrm{fm}^{-2}$ and $\mathrm{En}, \mathrm{Ep}=2-8 \mathrm{MeV}$ (see figure 9 ), effects due to meson exchange currents, isobar currents and relativistic effects are important. Two Multi-Wire Proportional Counters (MWPC) planned for BigBite will allow a readout rate of several MHZ. This will enable running the experiment at high luminosity of up to a few $10^{37} \mathrm{sec}^{-1} \mathrm{~cm}^{-2}$, at which the total singles rate in BigBite will be about $3 \mathrm{MHz}$.

\subsubsection{E01-014: Precision Measurement of Electro-production of $\pi^{0}$ near Threshold: A Test of Chiral QCD Dynamics.}

The dynamics of the pion-nucleon system are set at low energy by chiral symmetry [9]. One can test the convergence of the chiral expansion through a precision measurement of $\mathrm{p}(\mathrm{e}, \mathrm{e} \mathrm{p}) \pi^{0}$ near threshold. In this regime only the Swave amplitudes $\mathrm{E}_{0+}$ and $\mathrm{L}_{0+}$ survive. Together they form a "reduced" S-wave cross-section. The $96 \mathrm{msr}$ solid angle acceptance and large momentum acceptance of the BigBite spectrometer will allow new measurements to be made with high statistical precision (1\% at peak cross sections) and to be compared with theoretical predictions. Systematic errors will be minimized since the BigBite spectrometer can measure the entire proton angular distribution within a single kinematic setting (see figure 10). 


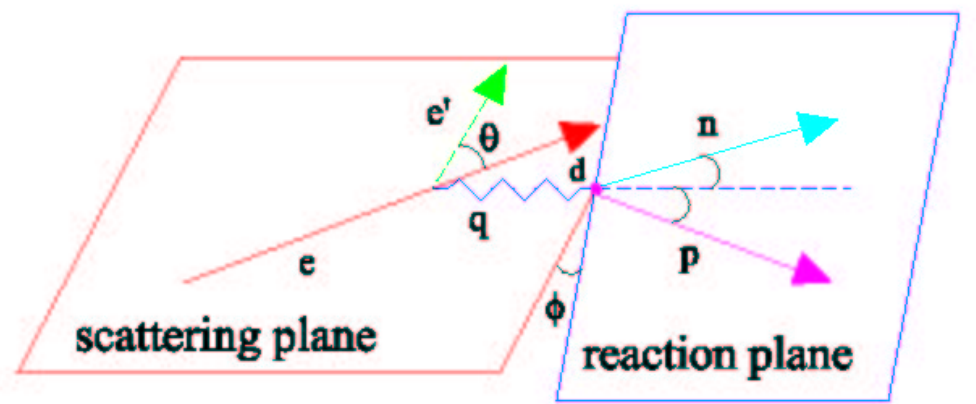

Figure 9: E02-101 Experimental kinematics.

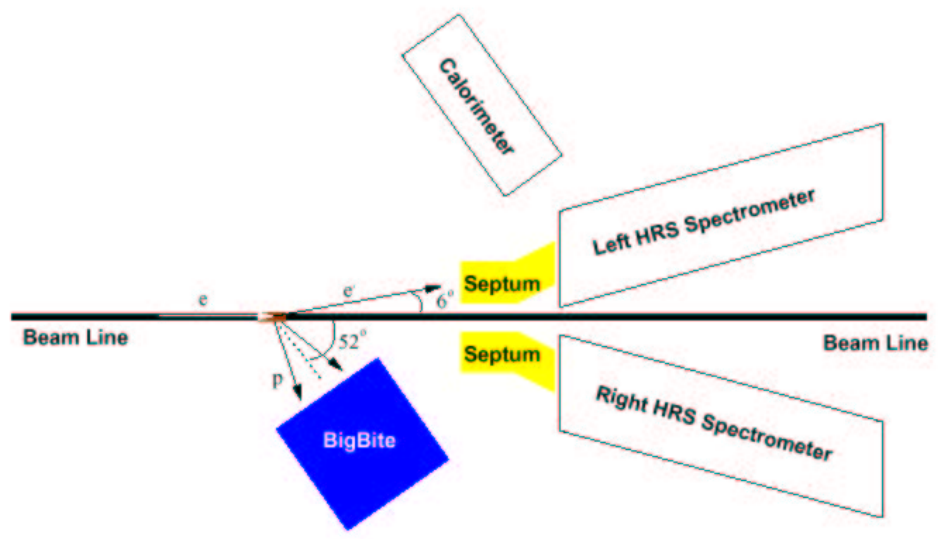

Figure 10: E01-014 Experimental set-up. The two $\gamma$ emitted by the $\pi^{0}$ decay will be detected in the calorimeter. The outgoing electron will be detected using the two HRSs and the outgoing proton will be detected with BigBite. 


\subsubsection{Summary}

The experiments described above give a short inventory of the different physics and requirements in high luminosity operation of large solid angle scintillators arrays in Hall A. In the next section we will discuss briefly the principle of operations, and in the forthcoming chapters we will present the design, construction and performance of such scintillator arrays.

\subsection{Scintillators Operation}

Scintillation detectors are a fundamental tool in experimental nuclear physics. All the experiments described above use scintillator arrays as part of their detection apparatus.

A typical scintillator counter (see figure 11), consists of a block of a scintillator material coupled to light-guides which are in turn coupled to photomultiplier tubes (PMT). The passage of a particle through the scintillating material causes emission of light. A scintillation counter (scintillator) can serve two functions: determine the time that a particle hit the detector and measuring energy deposited by incident particles (the amplitude of the produced signal in first approximation is proportional to the energy deposited). A charged particle passage, causes light emission (typical wave length of 340-490 nanometer, see figure 12) originating from a direct excitation of the scintillator molecules to a higher energetic states. Neutral particles will emit light by scattering from a proton in the matter, which then travels in the material and excites the molecular energy states. The emitted light is not self-absorbed because of the differing shape of the excited and ground state energy levels as a function of inter-atomic spacing.

Both inorganic and organic scintillators have been discovered and have different scintillation process. Inorganic crystals (like NaI, CsI) are usually grown with small admixture of impurity centers. The positive ions and electrons created by the incoming particle, diffuse through the lattice and are captured by the impurity centers. Recombination produces an excitation and light is emitted upon the return to the ground state. The light output is typically large in an inorganic scintillator, but the electron migration through the crystal lattice results in a pulse of long duration $(\sim 1.5 \mu \mathrm{sec})$.

Organic scintillators have smaller light output, with faster time response. The advantages of the organic materials include good transparency to their own radiation, short decay times, emission spectra well matched to photomultipliers and easy adaptability. Organic scintillators can be further subdivided into organic crystals, liquid scintillators, and plastic scintillators.

Plastic scintillators typically have density of $\sim 1.0 \mathrm{~g} / \mathrm{cm}^{3}$ and an index of 
refraction of $\sim 1.6$. Plastic scintillators have fast response time, making them useful for timing and triggering applications. In this work we will focus mainly on the use of plastic scintillators.

The amount of light produced for a given energy loss is not constant but depends on the production of quenching centers. These are activated molecules raised to excited vibrational levels. The number of produced photons roughly follows the equation:

$$
n=\frac{n_{0} d E / d x}{1+B d E / d x}
$$

were $\mathrm{B}$ and $n_{0}$ are constants and $\mathrm{dE} / \mathrm{dx}$ is the ionization energy loss.

The photon yield of a typical plastic scintillator is approximately equal to 20000 primary photons per $1 \mathrm{~cm}$ passage of a charged particle in the scintillating material.

Light emission from a scintillator is not instantaneous but has a time distribution [10]. This distribution is characterized by a rise time and a decay time. The random excitation of scintillator material leads to an exponential time dependence for photon emission.

$$
n(t)=k\left(1-e^{-t / \tau}\right) .
$$

$\tau$ - the typical decay constant, is about 5 - 50 nanoseconds.

Any large scintillator is affected by attenuation of the scintillation light traveling through the counter. The distance for the light intensity to fall to $1 / \mathrm{e}$ of its initial value is called the attenuation length, for plastic scintillators it is typically on the order of $1 \mathrm{~m}$.

Light collection is accomplished by coupling a light guide to the scintillator and connecting the light guide to a PMT. Light guide effectiveness is characterized by the fraction of light collected, the propagation time between light emission and light incidence on the PMT and by the width of the pulse created (the spread of the arrival times). The light guide characteristics depend on its shape and the material it is made from.

\subsubsection{Photomultiplier Tube}

The PMT converts the light from the scintillator into an electric pulse [11]. Light incident on a PMT window, passes through it and hits a photo-cathode, which releases electrons. The electrons are accelerated and multiplied by a series of dynodes (see figure 13), until they reach an anode were they are collected.

PMTs are characterized by several parameters : 


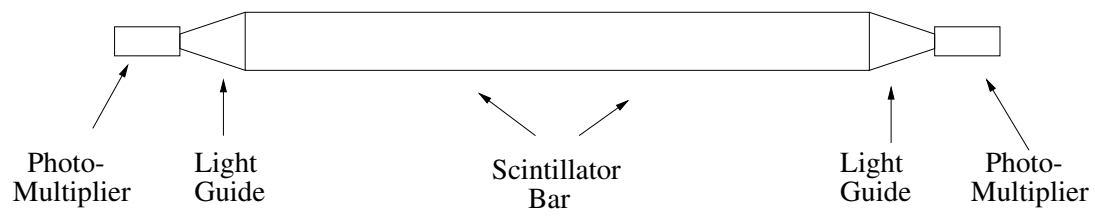

Figure 11: A schematic description of a BigBite scintillator.

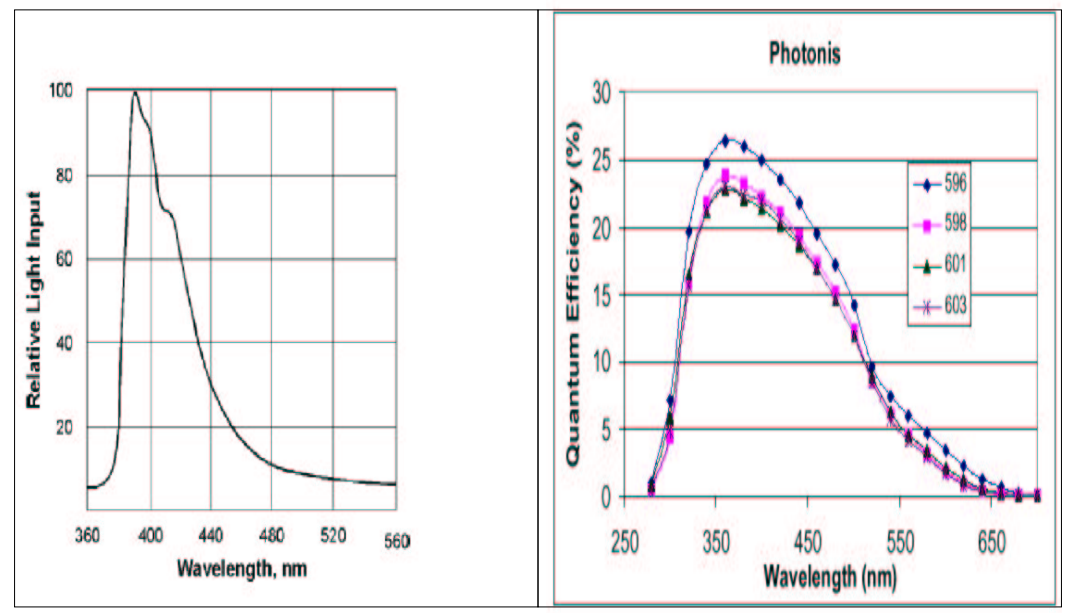

Figure 12: Emission and Quantum efficiency spectra Left - Light emission spectra for a plastic scintillator (BC414). Right- Quantum efficiency spectra, for different Photonis PMTs (different curves). 


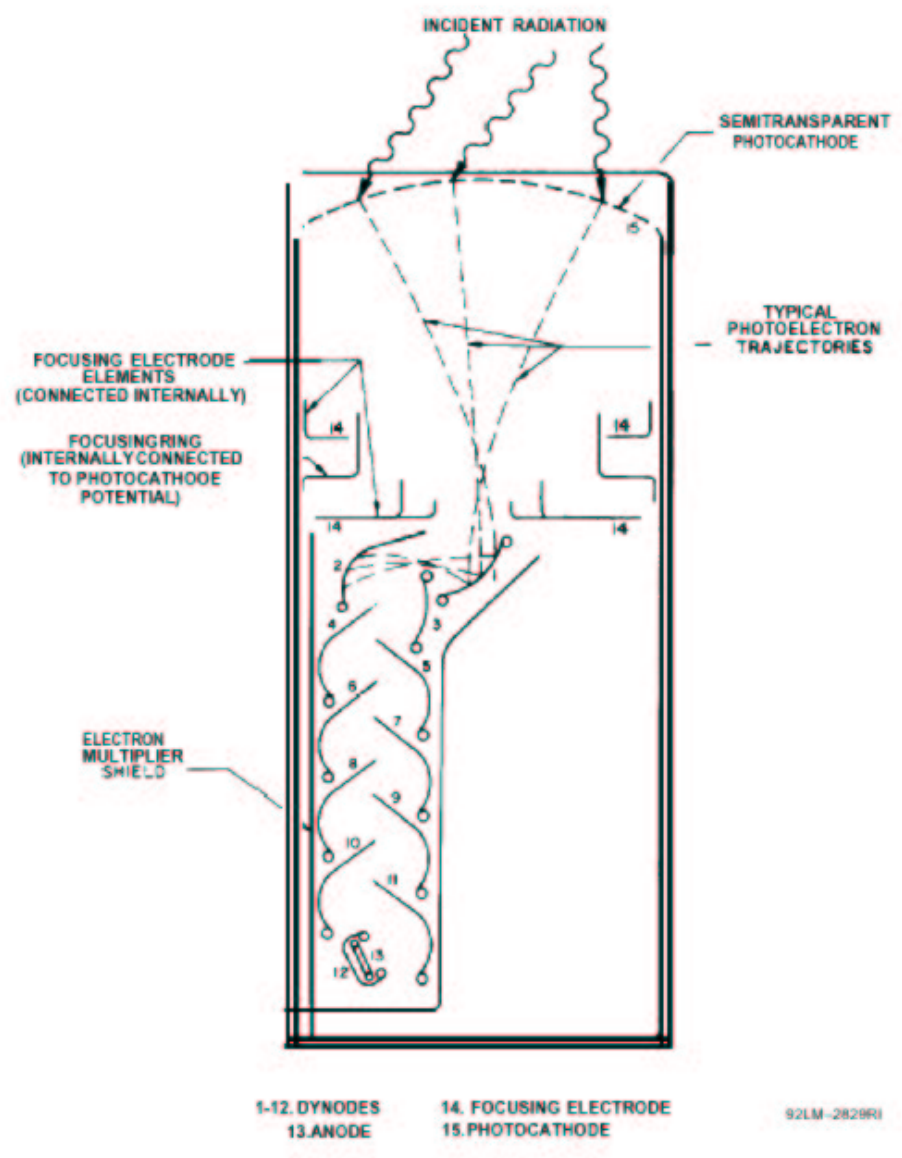

Figure 13: A schematic description of a PMT.

The different dynodes anodes and focusing electrode elements are shown. 
Quantum Efficiency. Defined as the ratio of the number of photoelectrons emitted by the cathode to the number of photons incident on the photocathode (usually on the order of 20\%). See figure 12-Left for typical spectra.

Window Material. The type of material used for the PMT window affects the transmitted light spectra and will be selected to match the light frequencies produced in the scintillator, and the photo-cathode sensitivity profile (see figure 12).

\section{Number of Dynodes.}

Gain. The ratio of the anode current to the photo-cathode current (varies as a power of the PMT base supply voltage ).

Response Pulse Width. FWHM of the anode current pulse delivered in response to a $\delta$-function light pulse.

Rise Time. The time required for the anode current to increase from $10 \%$ to $90 \%$ of its final value in response to a step function input.

Transit Time. The interval between arrival of a light pulse at the photocathode and the arrival of a corresponding electric pulse at the anode.

Time Resolution or Jitter. Standard deviation of the probability distribution for the transit time.

Dark current The background current signal produced when no light is entering the PMT.

All these characteristics determine the design and construction of scintillator arrays, to be further discussed in the following chapters.

\subsubsection{Momentum and position measurement}

We plan to use scintillators to determine particle momentum by recording the Time-Of-Flight (TOF) of the particle over a known distance using TimeDigitized-Converter (TDCs).

For a large detector, with two PMTs on both sides, the TOF of a particle can be calculated independent of the hit position along the detector, by averaging over the Left and Right PMT times:

$$
T=[T(l)+T(r)] / 2 .
$$

Another common application is position measurements along a scintillator bar by using time difference between $\mathrm{T}(\mathrm{l})$ and $\mathrm{T}(\mathrm{r})$.

$$
\text { Pos }=[T(l)-T(r)] / 2 * c^{\prime} .
$$


where $c^{\prime}$ is the speed of light propagating through the scintillator $\left(c^{\prime} \sim c / n\right.$, where $\mathrm{n}$ is the index of refraction in the scintillator ). For typical plastic scintillators c' $\sim 20 \mathrm{~cm} / \mathrm{ns}$.

\subsubsection{Expected resolution}

The expected resolution in TOF and position is determined by the time resolution. The time resolution is a function of a number of factors :

-Physical dimensions and geometry of the scintillator and the light guide.

-The scintillator response time.

-The photomultiplier tube parameters.

-Contribution from the fast electronics and TDCs

With the scintillator arrays we designed and built for Hall A, we achieved time resolutions of 0.1-1 ns as discussed in detail in the following chapters. 


\section{Scintillator planes for the BigBite spectrometer}

\subsection{BigBite spectrometer}

The new BigBite spectrometer in Jefferson lab's Hall A consists of a large acceptance, non-focusing dipole magnet and various detector planes. The large angular and momentum acceptance of BigBite will complement the two High Resolution Spectrometers (HRS) already present in Hall A, and will enable various third arm measurements that require large momentum and/or large angular acceptance (see previous chapter).

\subsubsection{Design and building of scintillator planes for BigBite}

This chapter will discuss the BigBite segmented scintillator planes for the SRC experiment (E01-015). For this setup, three different scintillator planes were designed, built and tested - the Auxiliary, the $\mathrm{E}$ plane and the $\Delta \mathrm{E}$ plane (see figure 14).

BigBite was designed to measure momentum and angular distribution of charged particles. Timing, hit position and energy deposited in the scintillators are all being used to measure the in-coming particles direction and momentum. A non-focusing magnetic dipole $(\mathrm{B} \sim 0.9 \mathrm{~T})$, is being used to curve the charged particles path before they hit the detector planes. The radius of curvature of the particle path in the magnetic field is proportional to the particles' momentum (see section 2.3 for the detailed calculation). Utilizing the segmented scintillator planes enables using the hit bar number and the timing differences between left and right PMTs to calculate hit position in three axis ([12]). The hit pattern information of each scintillator plane can be used to reconstruct the particle path inside the magnetic field and the corresponding particle momentum (size and direction). The particles' momentum is also calculated by measuring TOF along a known distance (i.e. between two scintillator planes or from the target to the E plane). The best momentum resolution is achieved by measuring the TOF between the target to the E plane, while the other methods are mostly used to filter background noise.

Particle Identification is done in two ways - by comparing the TOF and energy, or by measuring the energy deposited in the $\mathrm{E}$ and $\Delta \mathrm{E}$ planes. As their names suggest, the $\Delta \mathrm{E}$ and $\mathrm{E}$ planes are designed so that the $\Delta \mathrm{E}$ scintillators absorbs only a fraction $(\Delta \mathrm{E})$ of the particle initial energy, while the $\mathrm{E}$ scintillators are designed to absorb most or all of the particles' energy (see figure 42).

\subsection{The Auxiliary plane}

The Auxiliary plane (shown in figure 15) [13], consists of 56 scintillators. Each scintillator bar has dimensions $350 \times 25 \times 2.5 \mathrm{~mm}^{3}$ in and is coupled using a lightguide to a PMT in one end (see figure 16). The scintillators are arranged in a 


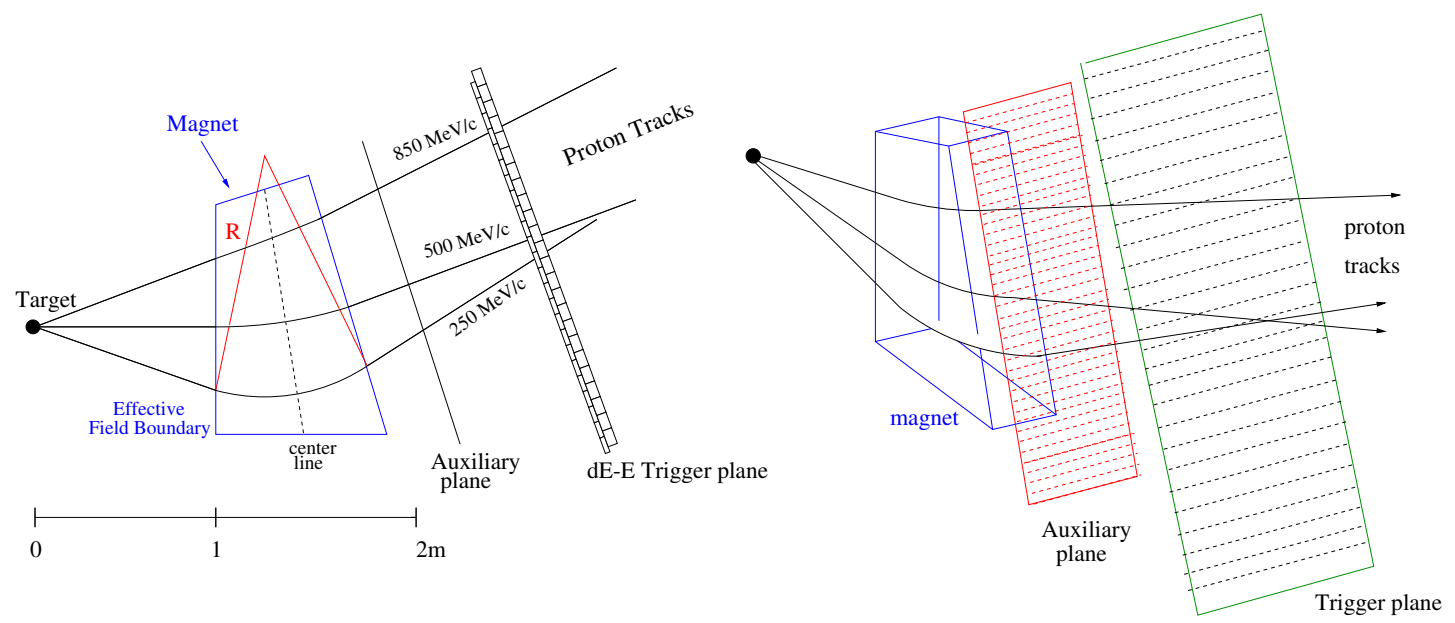

Figure 14: A schematic side view of BigBite, a few proton tracks are also shown.

staggered configuration. The PMTs are shielded using soft iron cylinders.

\subsubsection{The PhotoMultiplier Tubes}

The design is based on Hamamatsu-R1450, which is a $19 \mathrm{~mm}$ diameter, 10 stage linear focused tube. The gain is $5 \times 10^{6}$, the $\mathrm{QE} \sim 28 \%$ at $420 \mathrm{~nm}$, the rise time is $1.8 \mathrm{~ns}$, and the transit-time spread is $0.76 \mathrm{~ns}$. The PMT base voltage divider chain is configured for high rate. The first 3 stages are zener-dyode stabilized. These PMTs have been tested at $5 \mathrm{MHz}$.

\subsubsection{Scintillators}

The dominant consideration which determines the size/number of scintillators in the auxiliary plane is the singles rates and resolution. Our current estimate is about $5-10 \mathrm{MHz}$ for the full acceptance. With this design of 56 counters, we expect an average rate of about 100-150 KHz per counter;(scintillators along the planes, at positions which correspond to lower momentum, will have higher rate). The active area we wish to cover is $1400 \times 350 \mathrm{~mm}^{2}$, which determined the size of the 56 scintillators. The 56 - part segmentation also sets a hit position momentum reconstruction resolution, similar to the TOF momentum resolution. We allowed $1 \mathrm{~mm}$ gap between counters. The material is EJ-204, which was cut and diamond milled by ELJEN Technology. 


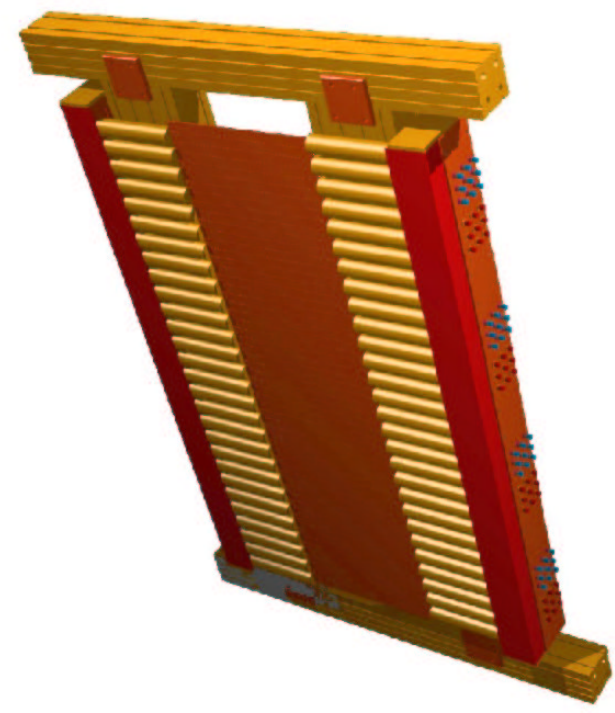

Figure 15: A CAD plot of the auxiliary plane.

\subsubsection{Light guides}

$85 \times$ (24 to 18$) \times 6 \mathrm{~mm}^{3}$ trapezian light guides were used. The scintillator (2 $\mathrm{mm}$ ) is glued to a grove in the center of the light guide face. The light guides are glued to the PMT. The material used for the light guides is BC- 800 produced by BICRON.

\subsubsection{Magnetic shielding}

The expected field at the Auxiliary plane position is roughly $0.02 \mathrm{~T}$ in the PMT axis direction. The design of the magnetic shield is based on soft iron cylinders of external diameter $42 \mathrm{~mm}$ and internal diameter of $23 \mathrm{~mm}$ (Rin/Rout $=0.76$ for each tube). The shield is extended more than two external radii beyond the photo cathode plane (see figure 17). We also used an inner mu-metal shield 0.8 mm thick, $95 \mathrm{~mm}$ long, with external diameter of $24.7 \mathrm{~mm}$. About 2-3 $\mathrm{mm}$ of the lower part of the iron cylinders were shaved off to enable to attach them to a common plate. The plates were then mounted on the same platform, as shown in figure 15. 

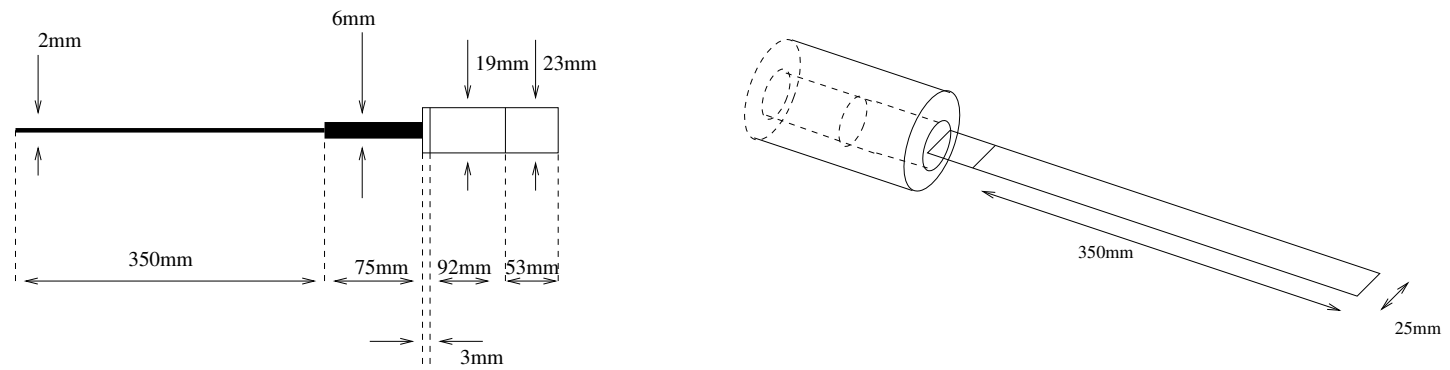

Figure 16: Schematic views of the an auxiliary plane scintillator.

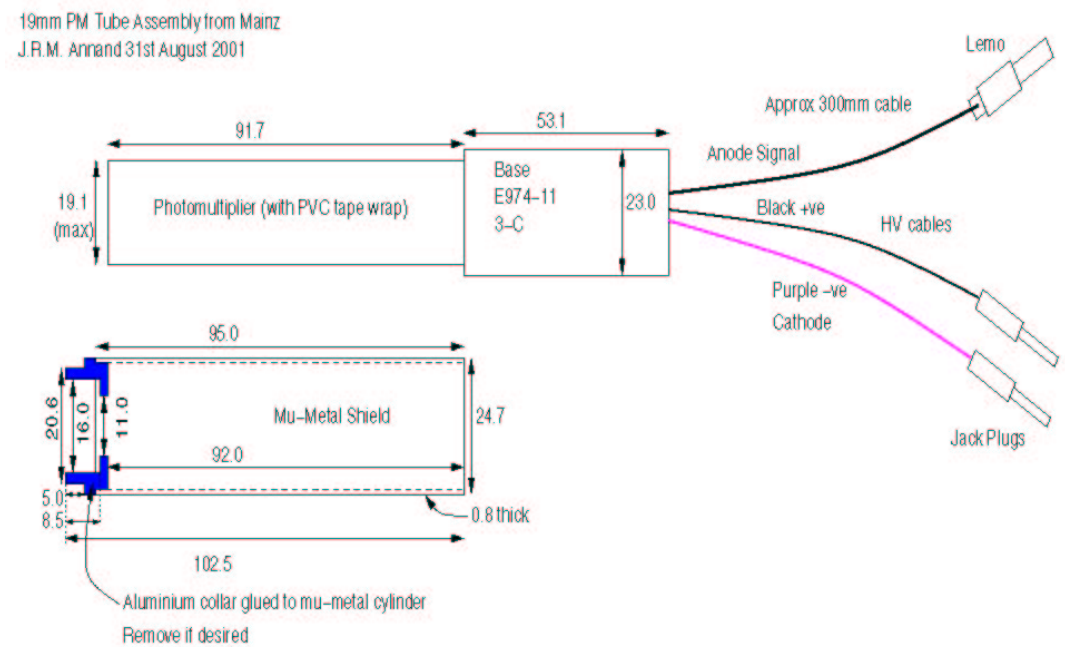

Figure 17: Schematic views of the an auxiliary light-guide, PMT and a soft iron magnetic shielding cylinder. 


\subsection{The Trigger plane}

The Trigger plane (see figure 18) is made up of two layers of segmented plastic scintillators. The first layer, $\Delta \mathrm{E}$ consists of $24,3 \mathrm{~mm}$ scintillator bars. The second layer E consists of 24, $30 \mathrm{~mm}$ bars. Each bar covers an area of 500x86 $\mathrm{mm}^{2}$ and is coupled to a PMT on both ends (see figures 19-22 for details).

\subsubsection{The Photomultiplier Tubes}

The selected PMT was Photonis-XP2262B , which is a $51 \mathrm{~mm}$ diameter, 12 stage linear focused tube. The gain is $\sim 6 \times 10^{7}$, maximum sensitivity is at 420 $\mathrm{nm}$, the rise time is $2.3 \mathrm{~ns}$, and the transit-time spread is $0.7 \mathrm{~ns}$. The base voltage divider chain is configured for high rate.

\subsubsection{Scintillators}

The dominant considerations which determines the size/number of scintillators in the trigger plane are again the singles rates and the resolution. The expected singles rate is about $5-10 \mathrm{MHz}$ for the full acceptance, which with a design of 24 counters, gives an average rate of about $200-400 \mathrm{KHz}$ per counter. The active area we wish to cover is $2100 \times 500 \mathrm{~mm}^{2}$, which determines the size of the 24 scintillators. Staggering the elements of each scintillator layer (see figure 19) gives a position resolution in the dispersive direction of $\pm 43 \mathrm{~mm}$ (one half of a single counter width). The scintillators are made out of EJ-204 material, which was cut and diamond milled by ELJEN Technology.

\subsubsection{Light guides}

Two $85 \times$ (24 to 18$) \times 6 \mathrm{~mm}^{3}$ trapezian light guides were glued to each scintillator bar. The material which was used for making these light guides is BC-800 produced by BICRON. The scintillators $(30 \mathrm{~mm}$ thick for the $\mathrm{E}$ bars and $3 \mathrm{~mm}$ thick for the $\delta \mathrm{E}$ bars) are glued to a grove in the center of the light guide face using Aljen optical cement (see fig 20). The light guides are glued to the PMT using a Dow containing silicon elastomer. 


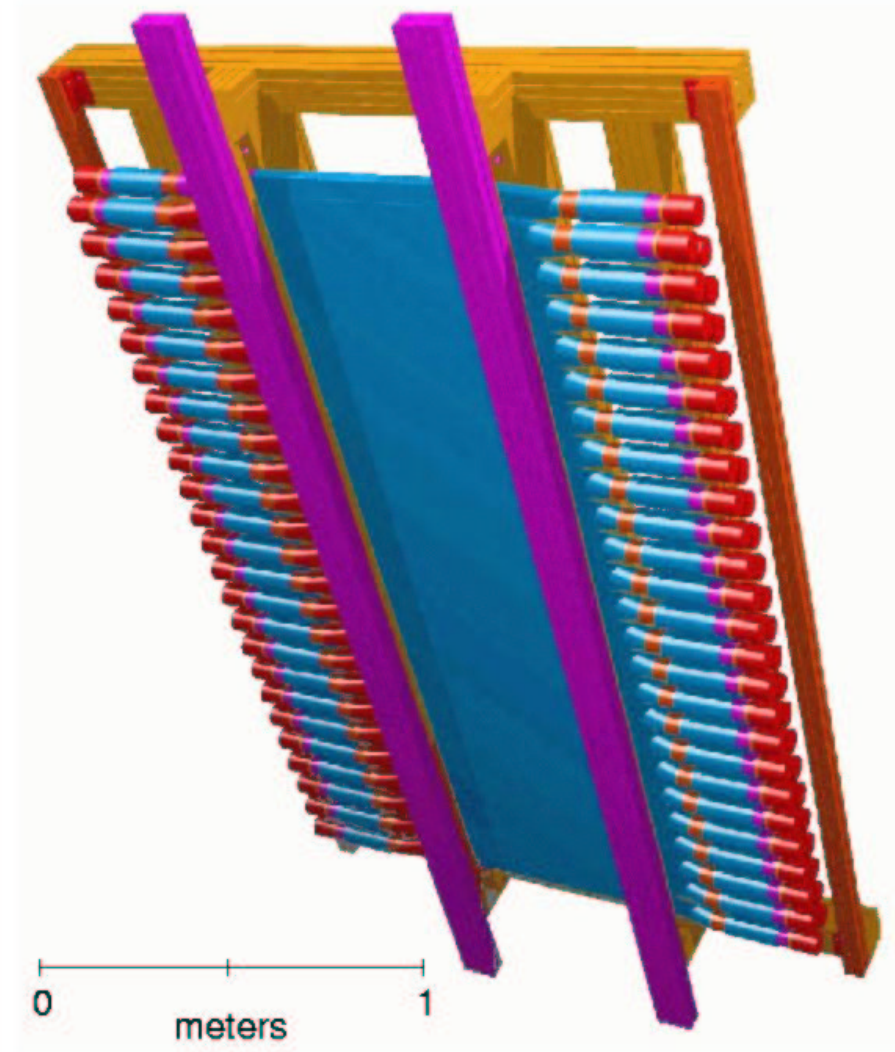

Figure 18: A CAD plot of the trigger $\mathrm{E}$ and $\Delta \mathrm{E}$ planes. 


\section{BigBite Focal-Plane Trigger Scintillators}
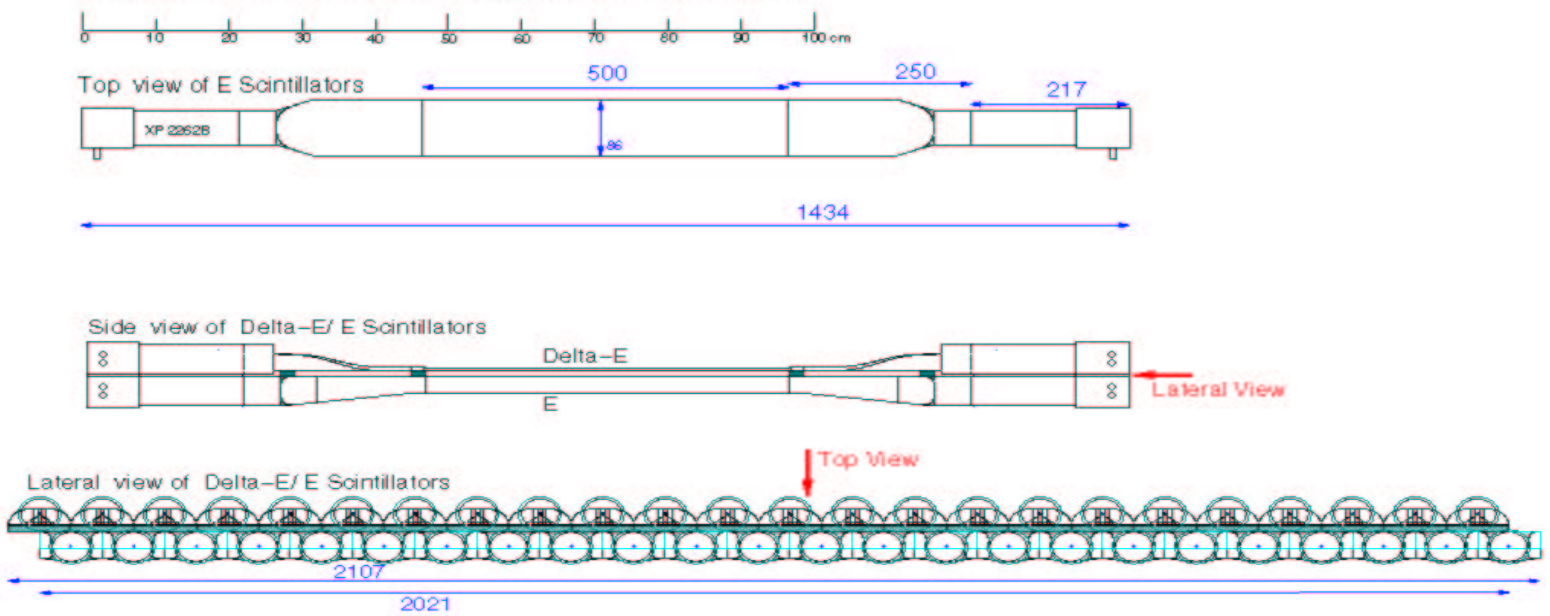

Figure 19: Schematic diagram of the $\mathrm{E}$ and $\Delta \mathrm{E}$ trigger plane scintillator.

"FISH-TAIL" LIGHT GUIDES FOR BIGBITE FOCAL-PLANE SCINTILLATORS Light guide to adapt $86 \times 30 \mathrm{~mm}$ to $52 \mathrm{~mm}$ diameter
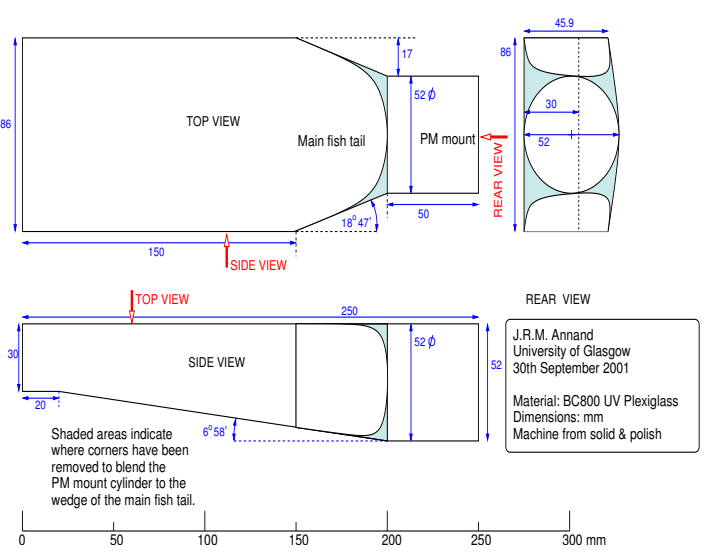

"FISH-TAIL" LIGHT GUIDES FOR BIGBITE FOCAL-PLANE SCINTILLATORS Light guide to adapt $86 \times 3 \mathrm{~mm}$ to $52 \mathrm{~mm}$ diameter

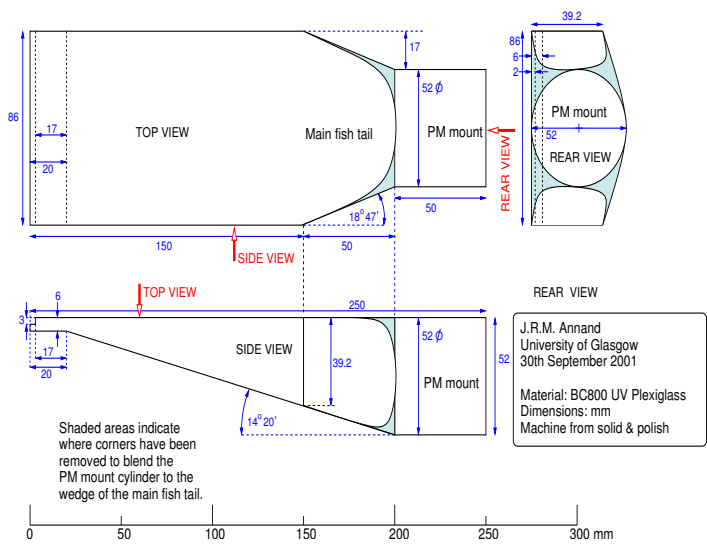

Figure 20: Schematic diagram of the $\mathrm{E}$ (left) and $\Delta \mathrm{E}$ (right) light-guides. 


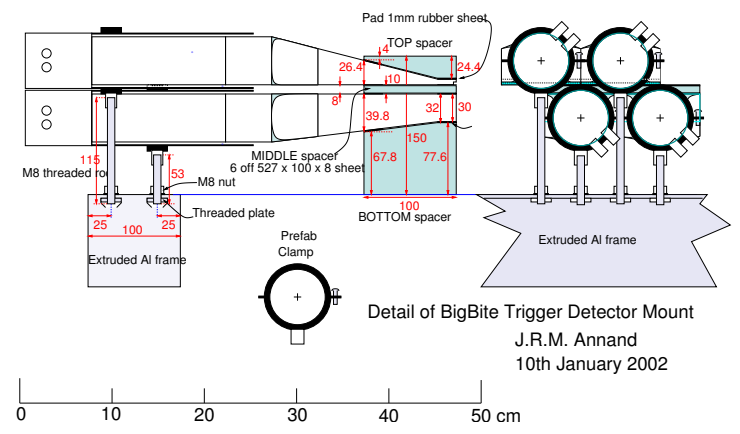

Figure 21: Schematic diagram of the $\mathrm{E}$ and $\Delta \mathrm{E}$ trigger plane scintillators mounting.

\subsection{Tests and Performance}

The BigBite detector package was tested using cosmic rays. The main focus of these tests was to determine timing resolution of individual scintillators and for the complete array. Separate tests were first done for each of the planes, followed by a complete detector package testing (without the magnet).

The high voltage (H.V.) was set for each PMT by using a radioactive source to calibrate the signal for each of the counters. The scintillator counters were then tested for time resolution and signal/noise separation. Data acquisition was performed using CODA [14] and analyzed using both ROOT [15] and PAW[16].

\subsection{Auxiliary plane testing}

The experimental setup that was used during the cosmic tests is shown in figure 23. Triggering of the readout electronics was performed using coincidence between two $5 \times 5 \times 200 \mathrm{~cm}^{3}$ scintillators centered above and below the auxiliary plane and perpendicular to the direction of the tested scintillators. This setup enabled us to test the entire auxiliary plane simultaneously [13].

\subsubsection{Analog-Digital-Converter (ADC) spectrum analysis}

The ADC spectrum shows a clear distinction between pedestal values and actual cosmic ray events, as seen in figure 24. The spectrum as seen with an oscilloscope is shown in figure 25 . The protons of $250-500 \mathrm{MeV} / \mathrm{c}$ that will be detected during the E01-015 experiment will create a signal even larger than the minimum ionizing cosmic rays. The channel difference between pedestal and the center of the distributed cosmic values was found to be in the range of 150-300 channels; note, the input to the fastbus ADCs was after a splitting panel that reduced the analog signal by a factor of 2 . The difference in channel number 


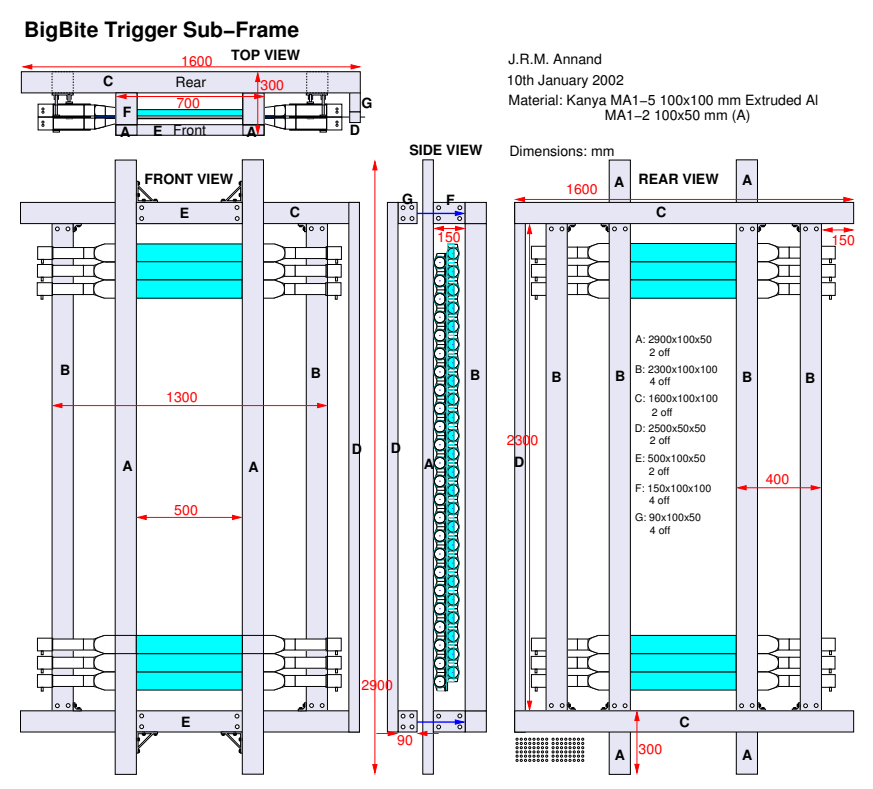

Figure 22: Schematic diagrams of the assembly of the trigger plane.

between the pedestal position and the position of a Gaussian fitted to the data for all the detectors is shown in figure 26. Measurements were performed with the cosmic trigger counters across the center and also near the even and the odd photomultiplier tubes. A time correction based on the ADC values was performed as detailed below.

\subsubsection{TDC spectrum analysis}

The time distribution for the detectors is influenced by both the time resolution of the tested detectors as well as that of the trigger detectors. Both of those resolutions together result in a Gaussian distribution with a typical standard deviation on the order of $1.2-1.5 \mathrm{~ns}$.

In order to determine the actual time resolution of the auxiliary detector only, we installed one of the spare counters in parallel to one of the counters in the array, between and perpendicular to the two cosmic trigger scintillators. We then looked at the time difference between these two counters. The trigger was the two cosmic ray detectors below and above the counters as shown in figure 23. The "start" time was always determined by a signal from the upper cosmic detector photomultiplier tubes. This distribution is shown in Figure 24. Assuming the time resolution of both of the tested auxiliary counters is equal, the width of the distribution $(\sigma=32$ channels $\mathrm{x} 50$ picoseconds per channel $=1.6$ 


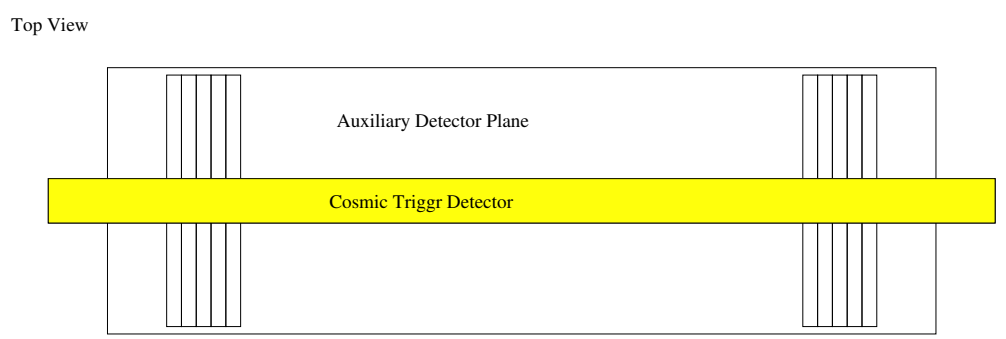

Side View

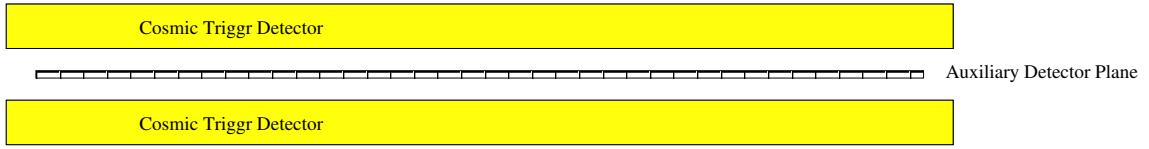

Figure 23: The setup used for testing the auxiliary plane with cosmic rays.

ns) is $\sqrt{2}$ times the detectors resolution ( $\sigma$ of about $1.1 \mathrm{~ns}$ ).

We also found a clear correlation between the ADC pulse height and the size of the TDC signal. An example is shown in figure 27 for counter \#40 and the extra counter we installed above it for the time resolution measurements. Similar behavior was found to exist in all counters. We fitted the TDC values as a function of the ADC value using the data from the central position trigger. The fit was to a second order polynomial as shown in Figure 27. We defined a corrected time as the time obtained for the counter corrected for the ADC value using the 3 fit parameters for that particular counter. The corrected time distribution under the same conditions as in figure 24 now has a $\sigma$ of 17.2 channels. Assuming equal contribution from the two equal detectors, it results in time resolution for each counter of :

$$
\sigma=17.2 * 0.05 / \sqrt{2}=0.6 \mathrm{~ns} .
$$

Figure 27. shows for counter 2 the time distribution for the central trigger before and after the correction. Figure 28 shows the corrected time distribution for the full array. The values are the TDC position and $\sigma$ for the "near", "far", and "center" triggers. 

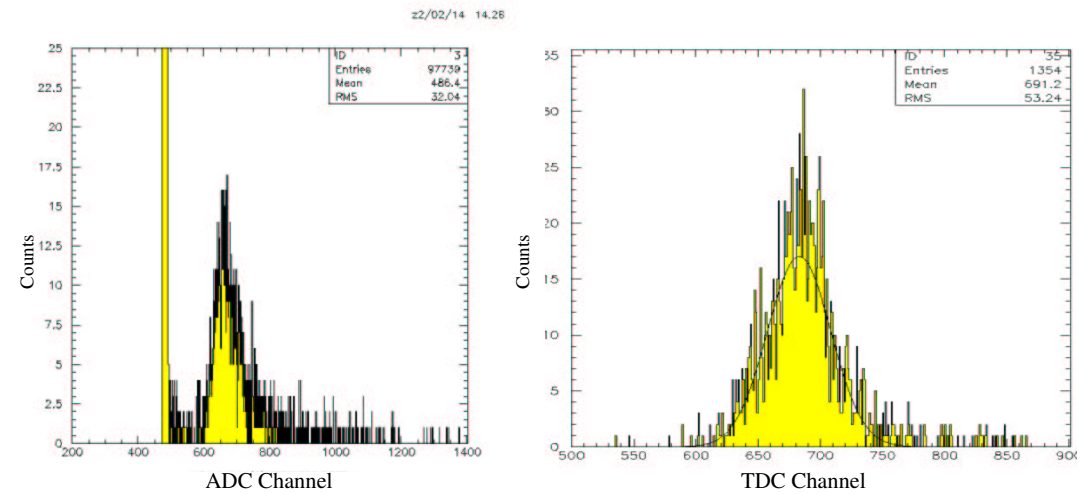

Figure 24: ADC (left) and TDC (right) spectrum for a typical auxiliary detector.

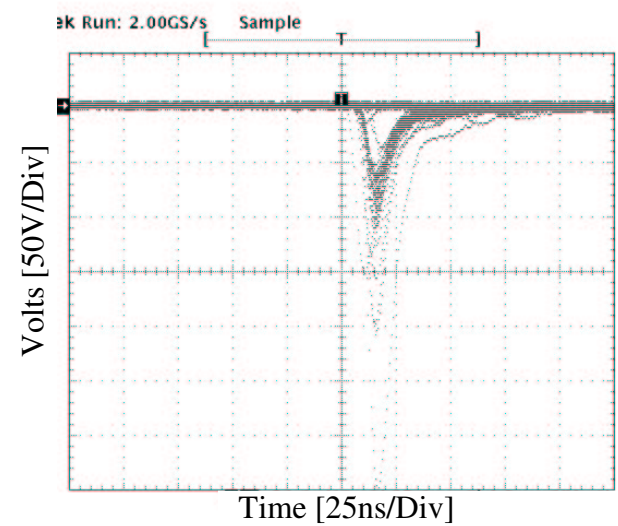

Figure 25: Cosmic ray events as viewed using an Oscilloscope.

For the same counter, as used to obtain the spectra shown in the previous figure. 

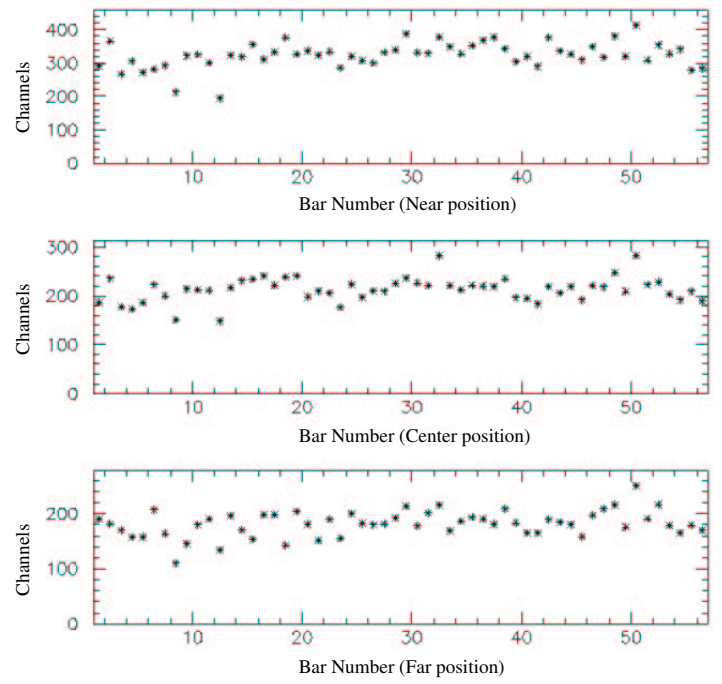

Figure 26: The difference between the pedestal and the cosmic peak positions for all the counters. Shown from top to bottom are the obtained values for "near"," center" and "far" positions. All are in ADC channel numbers.
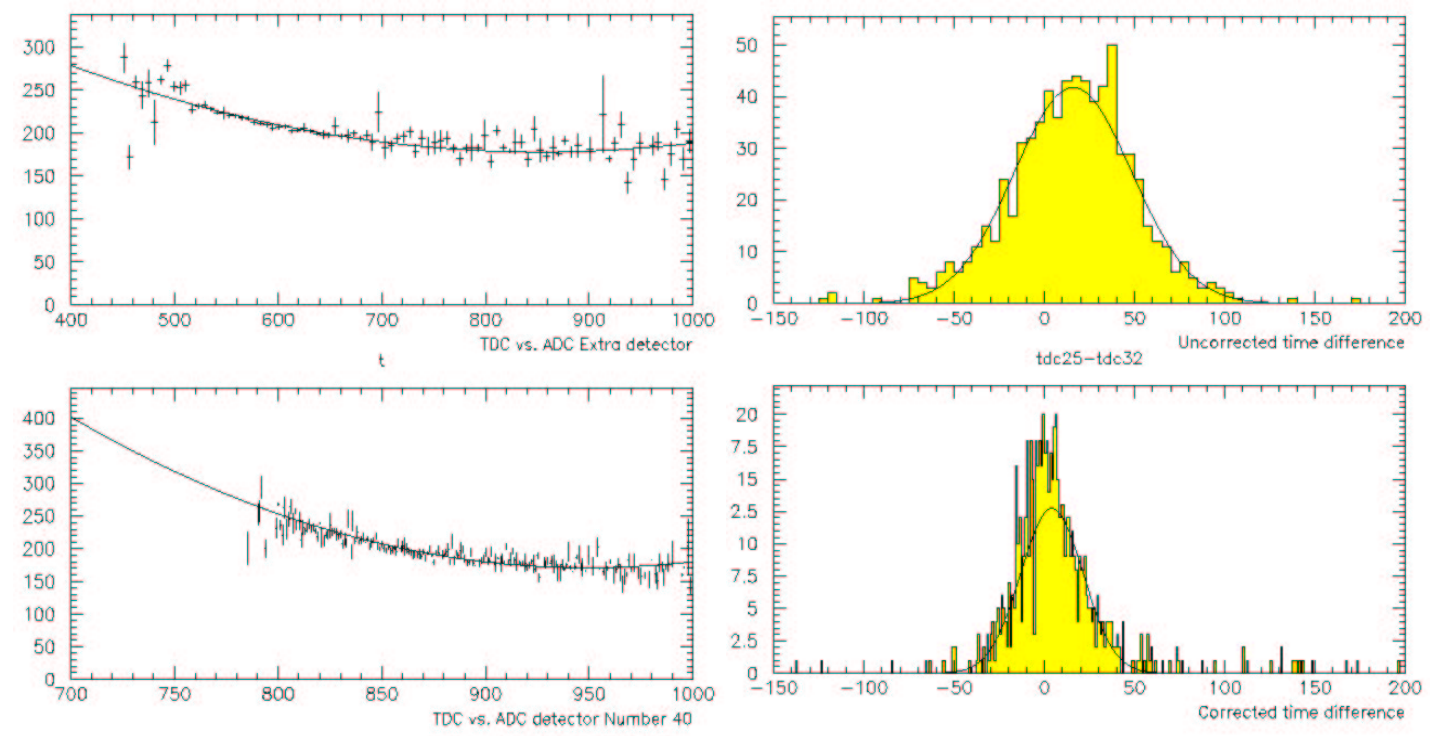

Figure 27: ADC correction.

Left- TDC vs. ADC for the two counters mounted one above the other. The cosmic ray trigger counters are above and below the center of the tested counters. Right- The time spectra of detector \#2 before and after the ADC correction done. 


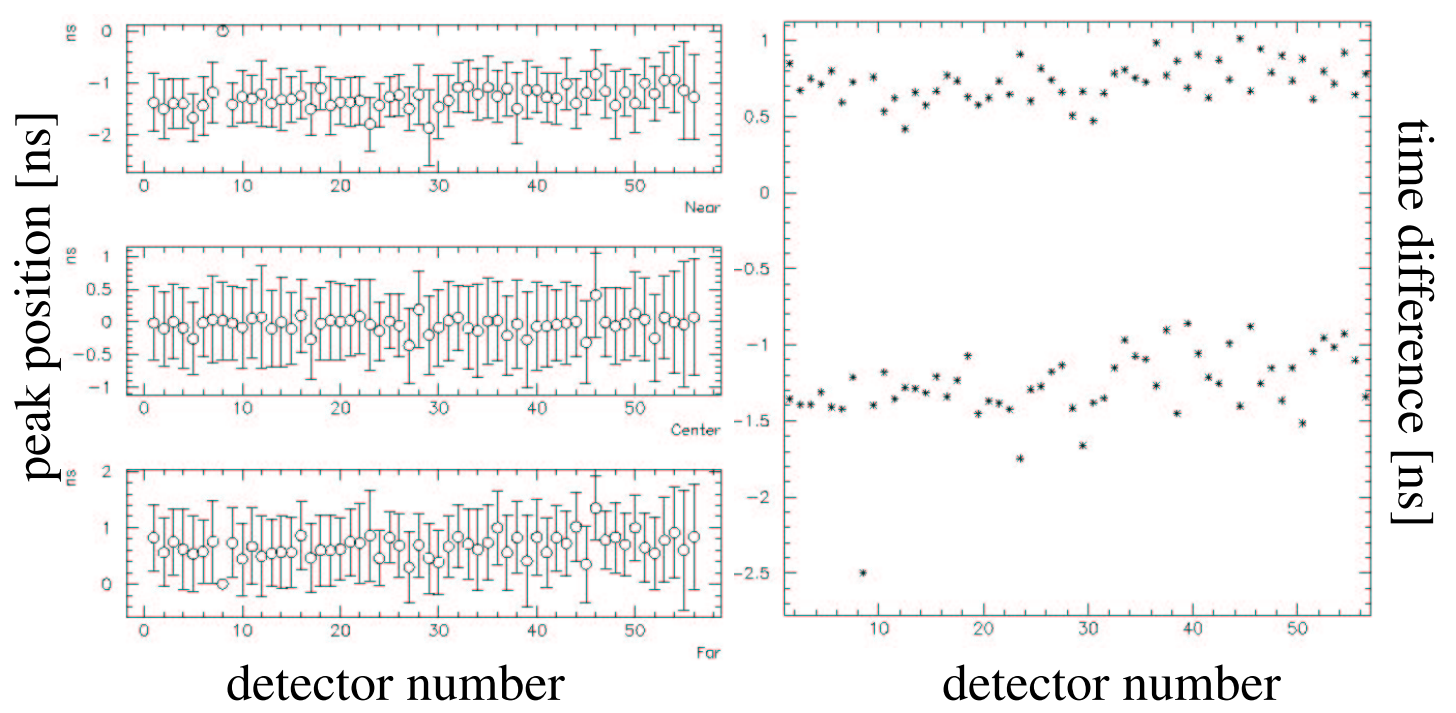

Figure 28: TDC peak

Left- The peak position of the time spectra of all the detectors after the ADC correction. The errors are the widths (standard deviations) of the fits. RightThe time difference between "far" and "center" as well as "near" and "center".

\subsection{Trigger plane testing}

The $\mathrm{E}$ and $\Delta \mathrm{E}$ trigger plane detectors were tested with cosmic rays in a similar way to the auxiliary plane [13]. The different setups used for the tests are shown in figure 29. The measured resolution for a single detector is effected by both the intrinsic resolution of the detector itself and that of the detector used to get the cosmic trigger:

$$
\sigma_{\text {calculated }} \sim \sqrt{\sigma_{\text {intrinsic }}^{2}+\sigma_{\text {trigger }}^{2}}
$$

In order to nullify the effects of the trigger resolution we subtracted Right side TDC signal from Left side signal. Assuming Left and Right resolutions to be equal we get:

$$
\sigma_{L-R} \sim \sigma_{L+R} \sim \sqrt{2} \sigma_{L} \sim \sqrt{2} \sigma_{R}
$$

The results for all the $\mathrm{E}$ and $\Delta \mathrm{E}$ bars are shown in Figures 30 and 31. Shown are the obtained standard deviation in sec.

In order to test TOF resolution between the auxiliary and the Trigger plane we placed the auxiliary plane beneath the Trigger plane at a distance of $80 \mathrm{~cm}$. To avoid introducing position dependence we used the two cosmic trigger bars placed above and beneath the two planes (Position 1). We selected only those 


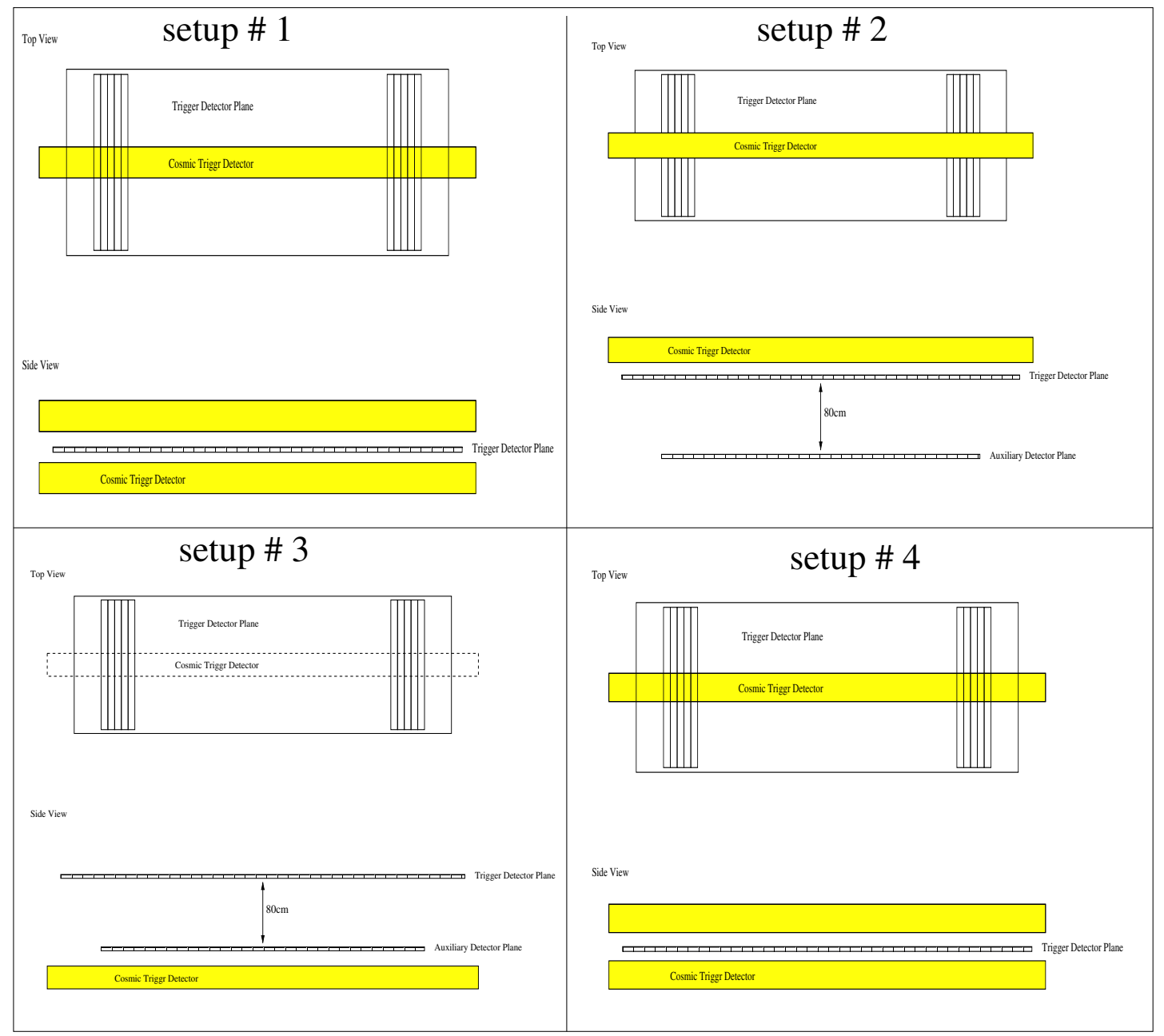

Figure 29: The different setups used for the trigger plane testing. 


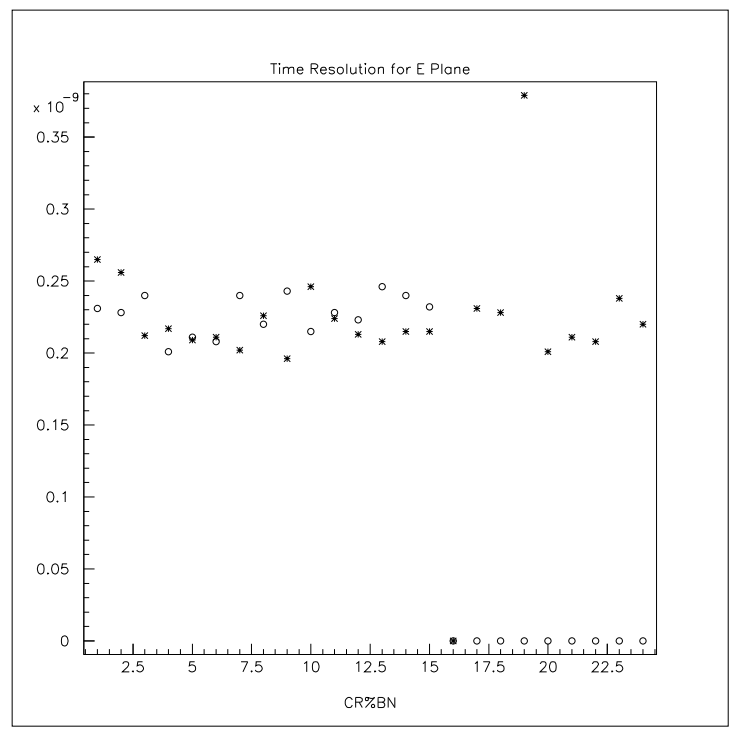

Figure 30: The calculated time resolution for the E counters.

The vertical axis are the standard deviation of the time distribution calculated as described by eq. 6 , in seconds. The horizontal axis is the detector numbers $(1-24)$.

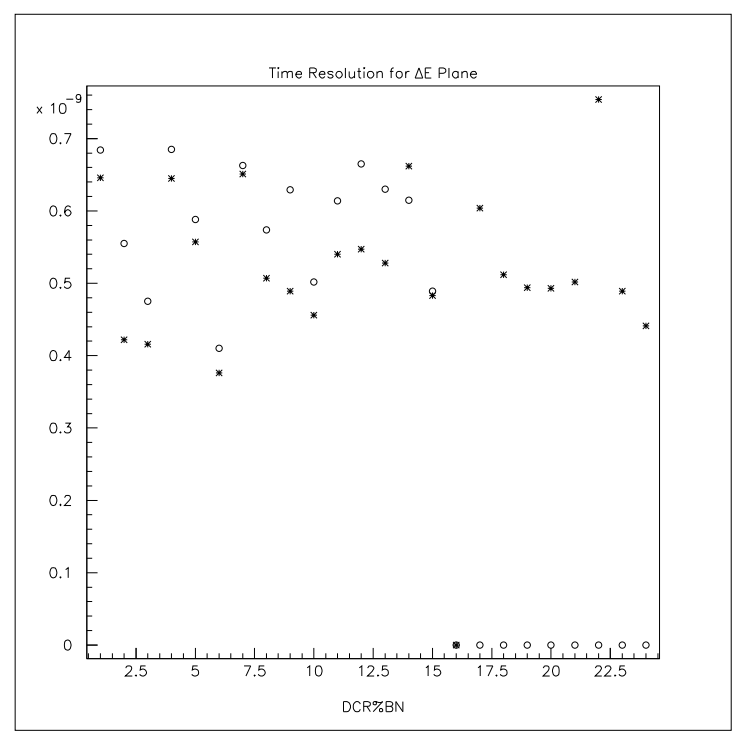

Figure 31: The calculated time resolution for the $\Delta \mathrm{E}$ counters.

The vertical axis are the standard deviation of the time distribution calculated as described by eq 6 , in seconds. The horizontal axis is the detector numbers $(1-24)$. 


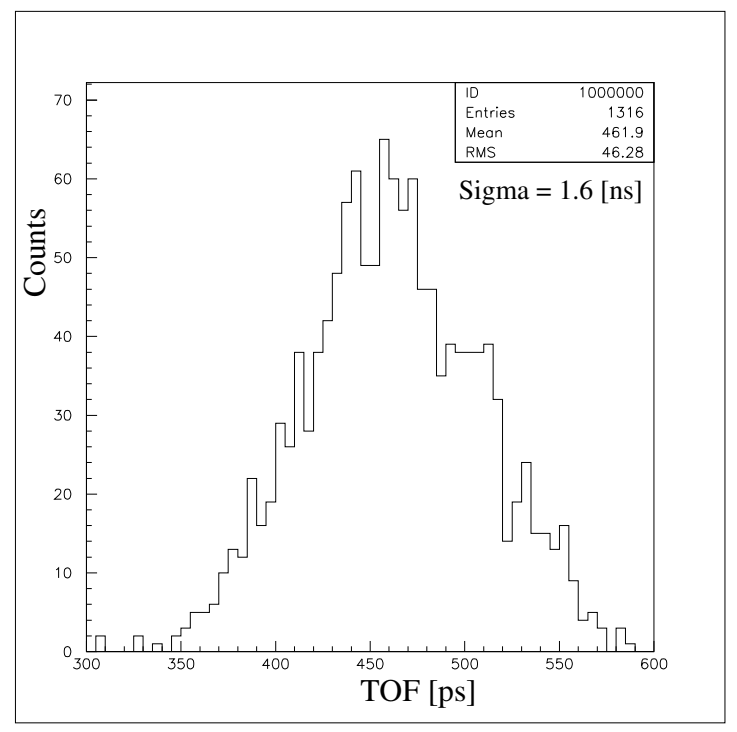

Figure 32: Typical TOF spectrum between an Auxiliary plane counter and a $\Delta \mathrm{E}$ counter before corrections.

events which described a specific path through both planes (i.e. hit exactly one Trigger and one Auxiliary bar). The resulted time spectra for channels 816 is shown in Fig32. The standard deviation for that measurement is $\sigma=1.6 \mathrm{~ns}$.

We then removed the bottom cosmic trigger bar and used only the top bar to trigger the DAQ producing hits across the whole length of the Auxiliary plane bars (position 2). The next step was to trigger from the bottom bar only giving us a definite position on the Auxiliary plane and hits across the entire length of the Trigger plane (position 3).

TOF measurements were corrected for each event, using the following steps:

- Adjust by software shift the intrinsic delay for each of the individual E and Auxiliary channels.

- Performed ADC correction for the time of Each E counter.

- Calculated the hit position along the E bar using $T_{l e f t}-T_{\text {right }}$.

- Calculated the corresponding hit position along the Auxiliary bar.

- Performed distance to PMT and ADC correction for the Auxiliary time.

- Used the corrected E and Auxiliary times to calculate the final TOF.

The resulted spectra of TOF/meter using the full array in setup 2, is shown in figure 33. The overall standard deviation of that TOF/meter is $0.84 \mathrm{~ns}$. 


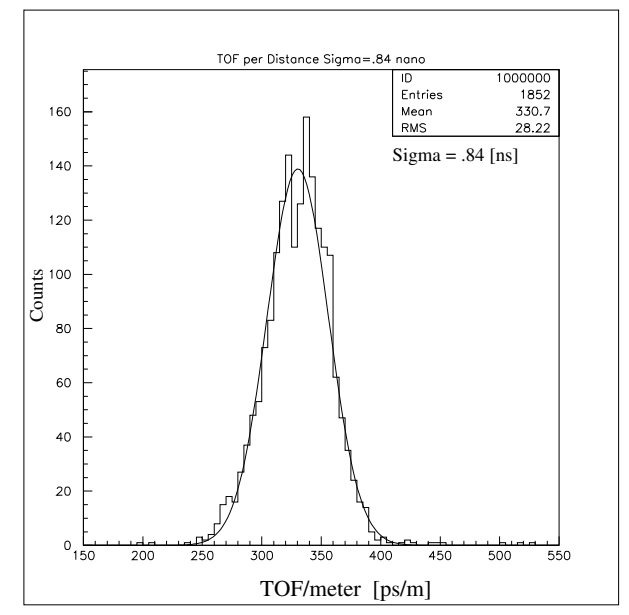

Figure 33: A typical TOF/Distance spectrum after all corrections (see text). The vertical axis is the number of counts and the horizontal axis is number of channels per meter. Each channel covers 35 ps. The standard deviation is $\sigma$ $=.84 \mathrm{~ns}$.

\subsection{Complete detector package testing}

A complete test with cosmic rays was conducted for the full detector package. The cosmic ray test was conducted for the $\mathrm{E}, \Delta \mathrm{E}$ and Auxiliary planes, with all the detector channels connected to the DAQ and using the final complete electronics setup. All together we had 152 channels in the ADCs and 152 channels in the TDCs. These tests where performed as a final check before the detector calibration in the beam. They enabled debugging of the counters, PMTs, electronics, and DAQ software. The detector package was mounted in its planned support frame, at an angle of $25^{\circ}$ to the vertical axis (see figure 14). The distance between the Auxiliary and Trigger planes is $\sim 1$ meter. TDC values for Auxiliary and Trigger Planes were corrected using coincidence measurements with a long fiber scintillator across the relevant plane.

The TOF/meter shown above was calculated as follows:-

$$
\text { TOF } / \text { Meter }=\frac{\left(T_{\text {Left }}+T_{\text {Right }}\right)-T_{\text {aux }}}{\text { Distance }_{\text {perevent }}}+C
$$

Where $T_{\text {Left }}$ and $T_{\text {Right }}$ are the left and right trigger PMT times after correction, $T_{a u x}$ is the corrected auxiliary PMT time and Distance is the calculated distance per event, between the two hit positions. 


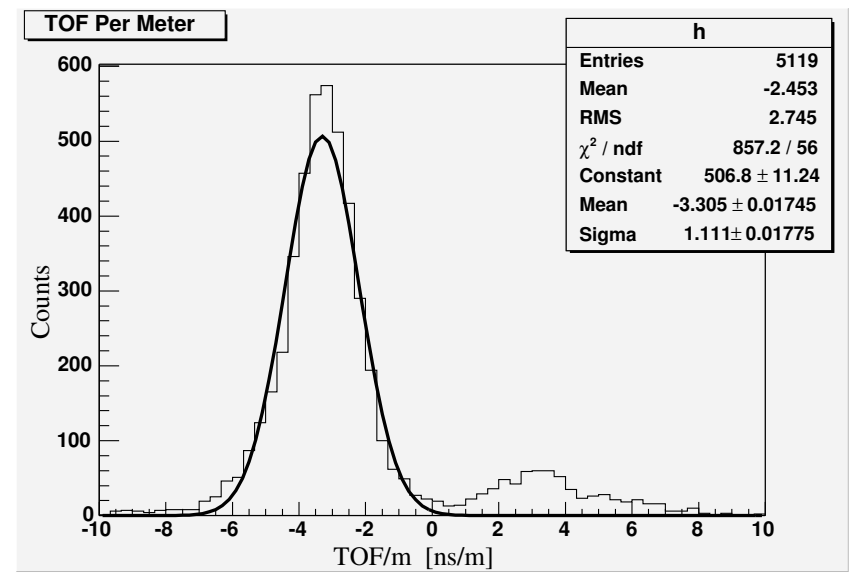

Figure 34: TOF/Meter [ns/meter] between the Auxiliary and the Trigger plane. The two separated peaks correspond to cosmic rays going from the Auxiliary to the trigger plane (right) and from the Trigger to the auxiliary plane (left). The difference between the peaks positions corresponds to twice the time that takes to cross the distance between the two planes, with the speed of light.

\subsubsection{Angular distribution of cosmic rays}

We have measured the angular distribution of cosmic rays, by calculating the incoming particle angle for each event. The angle is measured with respect to the vertical axis and is calculated as follows:-

$\Theta=\tan ^{-1}\left(\frac{\left(E_{\text {number }}-12\right) * E_{\text {width }}-\left(A_{\text {number }}-28\right) * A_{\text {width }}}{100}\right)\left(\frac{180}{3.1415}\right)+65$

Where $E_{\text {number }}$ and $A_{\text {number }}$ are the number of the bars that were hit on the E and Auxiliary planes. $E_{\text {width }}$ and $A_{\text {width }}$ are the E bar width $(8.6 \mathrm{~cm})$ and the Auxiliary bar width $(2.5 \mathrm{~cm})$.

The Cosmic ray angular distribution behaves as $\cos ^{2}(\Theta)$. The measured angular distribution was limited to cosmic rays going through both the Auxiliary and the Trigger plane. Due to the fact that there is no vertical "shadowing" between the two planes, no events appear with angles $<20^{\circ}$ (see figure 35).

\subsubsection{Measurement of the hit position across the Trigger Plane}

$$
\text { Pos }=\left(T_{\text {Left }}-T_{\text {Right }}\right) \cdot A+B
$$




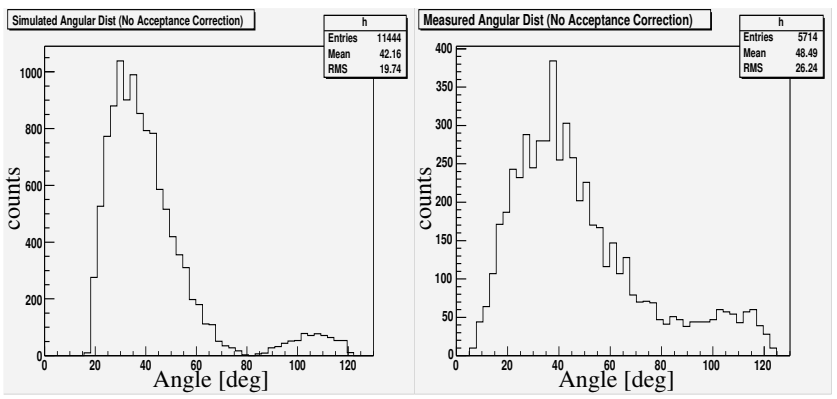

Figure 35: Cosmic rays angular distribution

Right - Cosmic rays angular distribution measured by the BigBite scintillator planes [counts/deg]. Left - Simulated angular distribution [counts/deg].

The angular distribution in angles $>90^{\circ}$ correspond to cosmic rays going from the auxiliary plane to the trigger plane.

Position measurements were taken along the Trigger plane counters. The measured position parameters (A and B in eq.9) for each counter were adjusted by fitting the measured position distribution to the expected spread $( \pm 25 \mathrm{~cm})$ and center position (0). Figure 36 shows the position distribution measured with (right) and without (left) a fiber trigger across the plane. The measured position resolution is $\sim 5 \mathrm{~cm}$, which agree with $\sqrt{2}$ times the timing resolution of each PMT counter (0.2 ns, as seen in figure 30), multiplied by the speed of light propagating inside the scintillator $(20 \mathrm{~cm} / \mathrm{ns})$.

\subsection{Simulations of BigBite Performances}

Various aspects of the BigBite spectrometer operation and functionality were simulated using different simulation tools. A complete scintillator array simulation was created using CERN GEANT4, which enables simulating the physics of the detector set-up and reconstruction of the output signals [12]. Incoming particle rates and angular and momentum distributions simulations were created using MCEEP, which is a Monte-Carlo simulation designed and built specifically for TJNAF Hall A [17]. The main questions which where answered using these simulations are -

1. What are the expected momentum and angular resolutions of BigBite with the scintillator array only (i.e no drift chambers)?

2. What are the expected angular and momentum acceptances of BigBite?

3. How can Particle Identification be performed?

4. What are the expected signal and singles rates? 

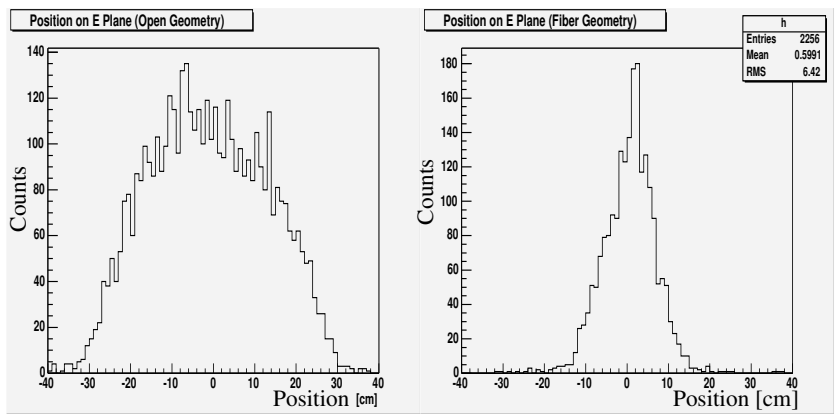

Figure 36: Measured position along counters

Right - position measured along the entire Trigger plane.

Left - Measured position with $1 \mathrm{~cm}$ wide fiber centered across the Trigger Plane and used as part of the trigger.

\subsubsection{Simulated momentum and scattering angle reconstruction with BigBite}

Scattering angle and Momentum reconstruction were simulated using a Geant4 simulation of BigBite [12]. The simulation assumes the existing detector package which consists of the Trigger and Auxiliary scintillator planes. All simulations were done assuming a point-like target. Different algorithms using both TOF and position information have been tested and compared.

\subsubsection{Momentum reconstruction using TOF}

Determination of the particle momentum by this method requires measuring the TOF for a known distance. Two different paths where tested for the reconstruction -

\section{a. TOF between the Auxiliary plane and the Trigger plane.}

We calculated the distance between the hit positions on the two planes based on the bar numbers that were hit on each plane, and the time signal from each PMT.

TDC signals:

$T_{l}$ - Hit time (ns) obtained from left PMT of the E counter

$T_{r}$ - Hit time (ns) obtained from right PMT of the E counter

$T_{\text {aux }}$ - Hit time (ns) of the Auxiliary PMT

$\mathrm{n}=\mathrm{E}+\Delta \mathrm{E}$ plane Bar Numbers. $\mathrm{n}=1$ to 48.

na $=$ Auxiliary Bar number. na $=1$ to 56 .

Xtc,Ytc,Ztc - Trigger planes center coordinates (mm) . 
Xac,Yac,Zac - Auxiliary planes center coordinates (mm) .

$\mathrm{V}=\mathrm{C} / 1.58$ - The speed of the light inside the scintillator media $[\mathrm{mm} / \mathrm{ns}]$.

Wt - The trigger plane bar half width $=45 \mathrm{~mm}$.

Wa - The auxiliary plane bar half width $=12.5 \mathrm{~mm}$.

$\mathrm{L}$ - The trigger plane bar length $=500 \mathrm{~mm}$.

The hit position calculation was done as follow :

On the Trigger plane:

$$
\begin{array}{r}
Z t=(n-24) \times W t \times \cos \left(25^{\circ}\right)+Z t c . \\
X t=(24-n) \times W t \times \sin \left(25^{\circ}\right)+X t c . \\
Y t=\frac{(T l-T r) L}{2}+Y t c .
\end{array}
$$

On the Auxiliary plane:

$$
\begin{gathered}
Z a=(n a-28.5) \times W a \times \cos \left(25^{\circ}\right)+\mathrm{Zac} \\
X a=(28.5-n a) \times W a \times \sin \left(25^{\circ}\right)+\mathrm{Xac} \\
Y a=Y t \times \frac{\sqrt{(X a-X e)^{2}+(Z a-Z e)^{2}}}{\sqrt{(Z t-Z e)^{2}+(X t-X e)^{2}}}
\end{gathered}
$$

The total path length from the Auxiliary plane to the Trigger plane :

$$
d=\sqrt{(X a-X t)^{2}+(Y a-Y t)^{2}+(Z a-Z t)^{2}} \quad(\approx 980 m m) .
$$

The total TOF with time correction for the light propagation to the PMT :

$$
T O F=\frac{T l+T r}{2}-T a u x-\frac{250-185 .+a b s(Y a)}{V} \quad n s .
$$

The particle relativistic momentum was calculated using the time and the distance :

$$
P=\frac{d / \text { tof }}{\sqrt{1-(d / \text { tof })^{2} \times 10^{-16}}} \times \frac{1.6726 \times 3}{1.6 \times 10^{-6}} \quad M e V / c .
$$


Based on resolution tests, we found that the time resolution of the Auxiliary plane is $0.8 \mathrm{~ns}$, and that of the E counters is $0.2 \mathrm{~ns}$. The major cause of uncertainty in this method is the Auxiliary plane time resolution. The results of simulation of mono energetic proton beams of different momenta with angular spread over the full acceptance, are shown in figure 37 .

\section{b. TOF between the Target and the Trigger plane.}

To obtain the time between the target and the trigger plane, we assumed an initial timing trigger is available from coincidence between the HRSs with a resolution of 1 ns and calculated the particle TOF along the entire path.

The entire path of the particle was divided to 3 segments -

-From the target to BigBite entry face:

$$
d 1=\sqrt{Z i^{2}+d^{2}} .
$$

-Curved path through the magnetic field:

$$
d 2=R \times a b s\left(\tan ^{-1}\left(\frac{X e-X o}{Z o-Z e}\right)+\tan ^{-1}\left(\frac{X i-X o}{Z o-Z i}\right)\right) .
$$

-From the point on the exit face to the Trigger plane hit point:

$$
d 3=\sqrt{(X t-X e)^{2}+(Y t-Y e)^{2}+(Z t-Z e)^{2}} .
$$

The total path is then given by :

$$
d=\sum_{i=1}^{3} d i(\approx 2700 \mathrm{~mm})
$$

$T_{H R S}$ - the time from the HRS's coincidence

$\mathrm{TOF}=\left(\frac{T l+T r}{2}\right)-\frac{250}{V}-T_{H R S}$

Particle Momentum -

$$
P=\frac{d / t o f}{\sqrt{1-(d / t o f)^{2} \times 10^{-16}}} \times \frac{1.6726 \times 3}{1.6 \times 10^{-6}} \quad \mathrm{MeV} / \mathrm{C}
$$

The simulated resulted standard deviations are summarized in Table 1. 


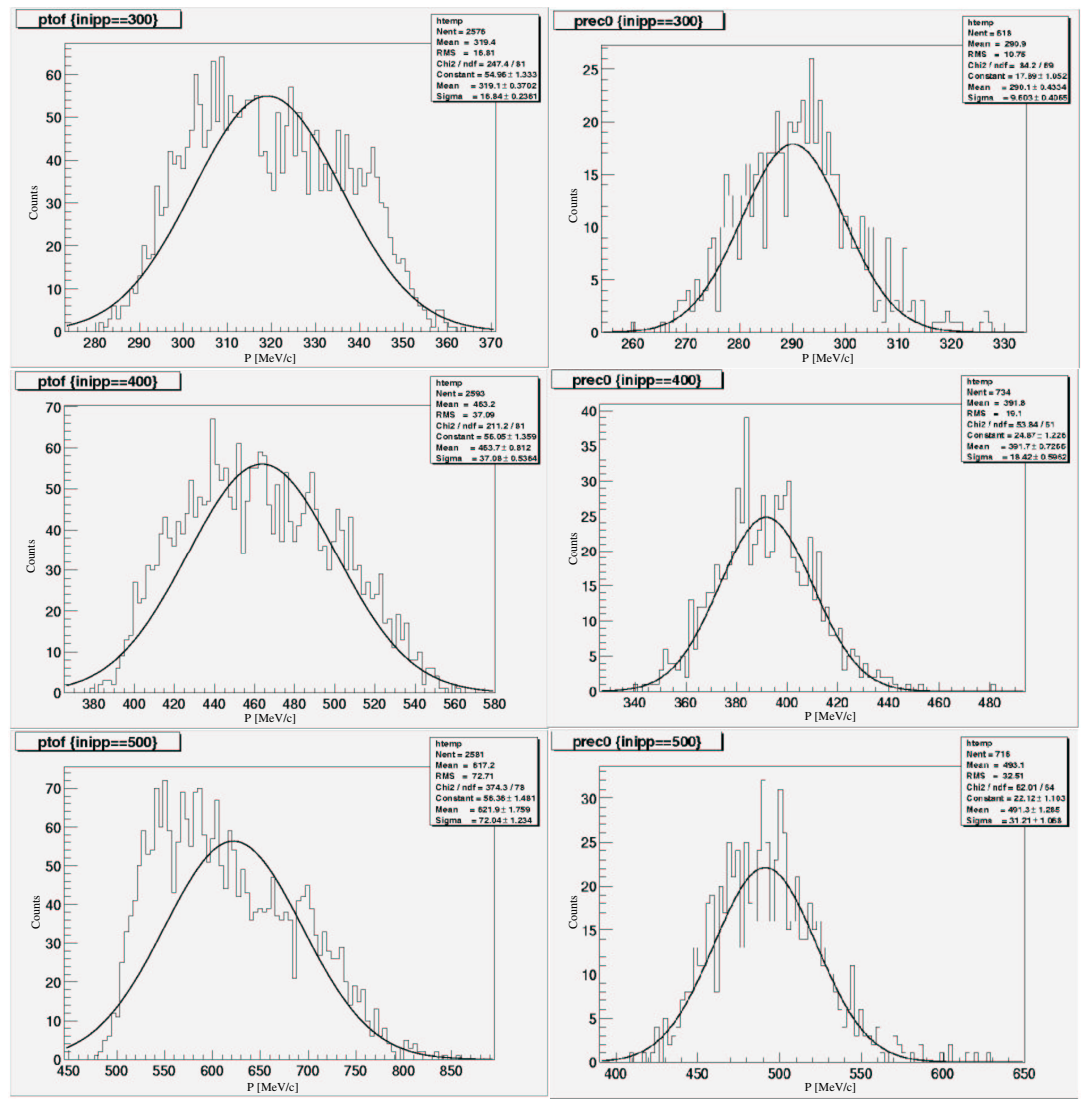

Figure 37: Reconstructed Momentum for protons with momentum of 300, 400 and $500 \mathrm{MeV} / \mathrm{c}$. The reconstruction was done using simulated TOF between the Auxiliary to trigger plane (left side) and simulated TOF between target to trigger plane (right side). 


\subsubsection{Momentum and scattering angle reconstruction using the Hit Position}

The particle momentum $\mathrm{P}$ and the scattering angle $\theta$ can be calculated using only the hit position in the two scintillators planes.

We have tried three different approaches in order to solve this problem : -Exact geometric solution for the path (calculation for each event).

-Values table, based on a specific fit for every hit pair.

-2 dimensional fit using MLP (Multi-Layer-Perceptron).

Exact solution -

We defined the following quantities ( see figure 35 ):

$(\mathrm{X} 0, \mathrm{Z} 0)$ the center coordinates of the circular path.

$\mathrm{axz}=(\mathrm{Zt}-\mathrm{Za}) /(\mathrm{Xt}-\mathrm{Xa})$ : incline of exit track.

ai $=\mathrm{Zi} / \mathrm{d}$ : incline of entry track.

$\mathrm{t} 2=\tan (25 \mathrm{deg}):$ detector and exit face angle.

bby $=817.4 \mathrm{~mm}:$ y length of BigBite.

bbx $=752.9 \mathrm{~mm}:$ base $\mathrm{x}$ length of BigBite.

$\mathrm{bbx} 2=$ bbx-bby $\times \mathrm{t} 2:$ top $\mathrm{x}$ length of BigBite.

$\mathrm{xm}=(\mathrm{bbx}+\mathrm{bbx} 2) / 4:$ mid point $\mathrm{x}$.

$\mathrm{xce}=$ bbx-bby $\times \mathrm{t} 2 / 2: \mathrm{x}$ of exit point at $\mathrm{z}=0$.

$\mathrm{xin}=0: \mathrm{x}$ of entry point in to BigBite.

We now find the intersection point of the exit vertex and the mid-field line.

$$
\begin{array}{r}
x t e m p=\frac{a x z \times X t-Z t+2 \times x i n / t 2}{a x z+2 / t 2} . \\
z \text { temp }=(x t e m p \times a x z)+Z t-(a x z \times X t) .
\end{array}
$$

The radius of the circular part of the trajectory is given by :

$$
R=\frac{\sqrt{1+a x z^{2}} \times\left(z e^{2}-2 \times z e \times z i-2 \times x i \times x e+x i^{2}+x e^{2}+z i^{2}\right.}{-z e-x i \times a x z+a x z \times x e+z i}(26)
$$

The solution for $\mathrm{P}$ and $\theta$ are then given by :

$$
P=B \times R \times 300, \quad \theta=\tan ^{-1}\left(\frac{z \text { temp }}{x \text { temp }+ \text { dist2target }}\right) .
$$

$\mathrm{B}$ is the Magnetic field in Tesla $(=0.92), \mathrm{R}$ is in $\mathrm{cm}$ and $\mathrm{P}$ in $\mathrm{MeV} / \mathrm{c}$. 


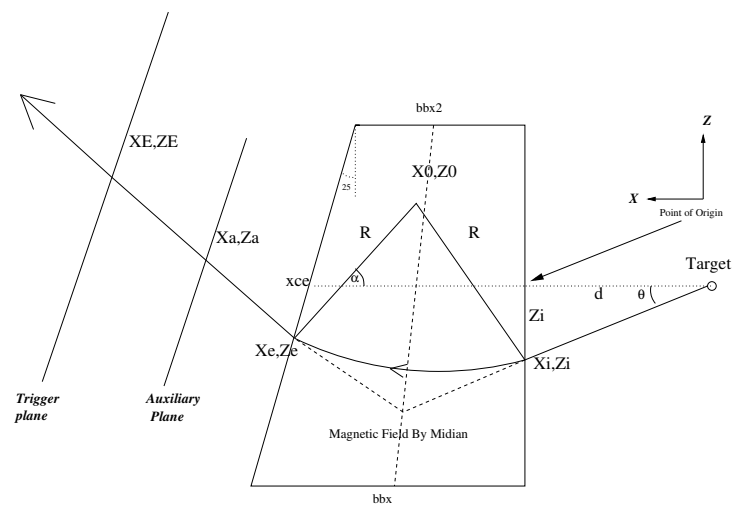

Figure 38: A Trajectory of a proton in BigBite. Simulated results with reconstruction based on hit position only, are shown in figures 39 and 40.

\subsubsection{Position and TOF combined algorithm}

In order to test ways to clean the background signals, an additional algorithm was examined. In this method, we used the consistency of the hit positions reconstructed momentum and the TOF reconstructed momentum (which has a smaller uncertainty but larger tails due to random hits), to clean background events.

We simulated $50 \%$ random hits by generating random times and applied a cut, by demanding that the difference between $\mathrm{P}$ (tof) and $\mathrm{P}$ (position) would be less then a specific limit, which was a fraction of $\mathrm{P}($ tof) (see figure 41).

$$
\mid P(\text { tof })-P(\text { position }) \mid \leq \mathrm{P}(\text { tof }) \times 5 \%
$$

\subsubsection{Particle Identification}

Particle identification can be done using time of flight and pulse height. Figure $42(\mathrm{~A})$ shows the expected flight time between the auxiliary and E planes, as a function of momentum, for $\mathrm{d}, \mathrm{p}, \mathrm{k}^{+}$and $\pi^{+}$. The projection of the flight times for momenta of $800-900 \mathrm{MeV} / \mathrm{c}$ in figure $42(\mathrm{D})$ shows reasonable separation between $\mathrm{d}, \mathrm{p}$ and unresolved $\mathrm{k}^{+}$and $\pi^{+}$. Notice that this separation is in momentum well above that which we expect in the SRC experiment $(\sim$ $600 \mathrm{MeV} / \mathrm{c}$ ). The time difference shows the more-or-less linear relation between incident angle $\theta$ (to the spectrometer central line) and the "measured" hit position, as shown in figure $42(\mathrm{c})$. 

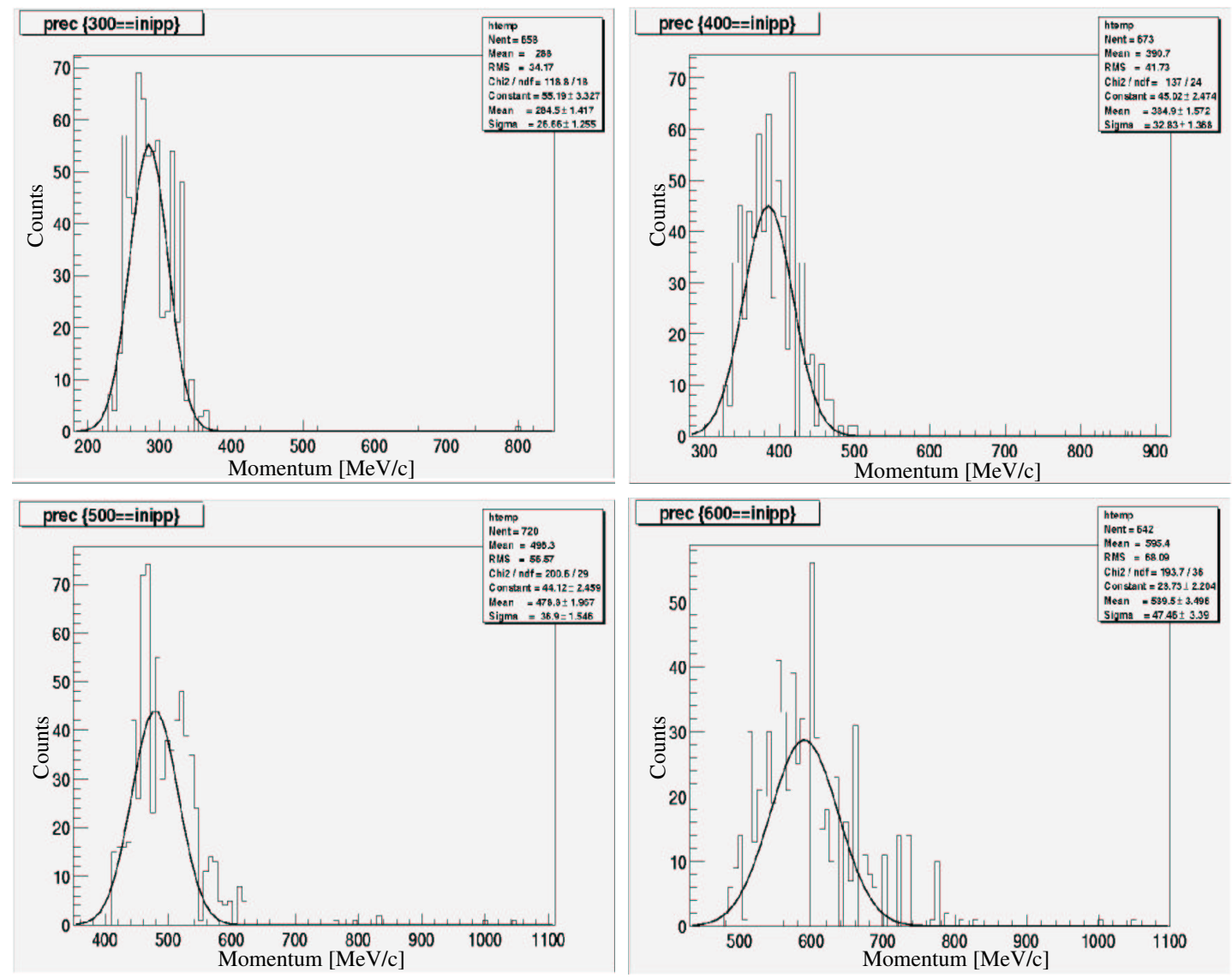

Figure 39: Momentum reconstruction using the analytical formula, based on hit positions. $\Delta \mathrm{P} / \mathrm{P}$ is $\approx 8 \%$ for the Momentum range $300-600 \mathrm{MeV} / \mathrm{c}$. 

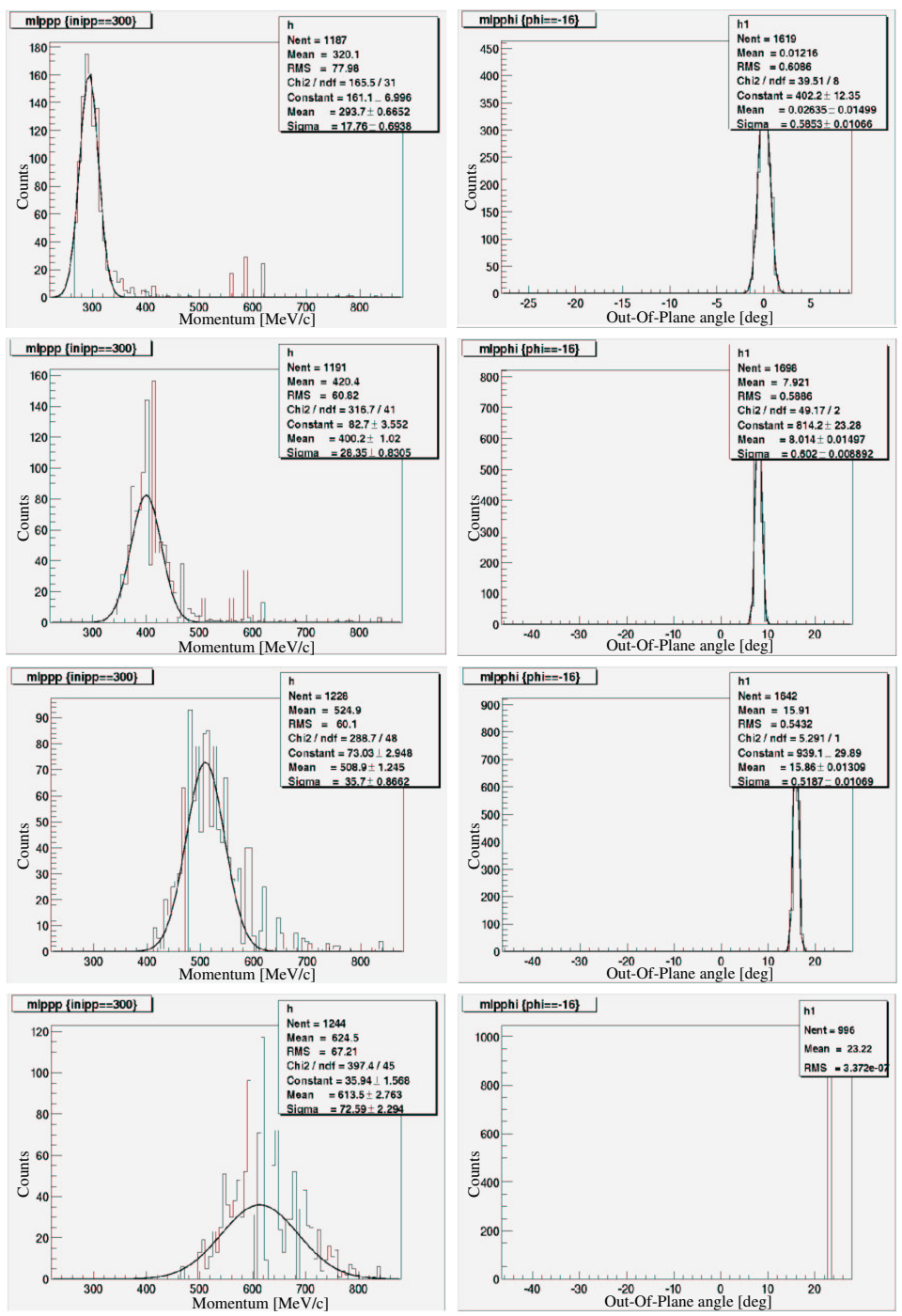

Figure 40: Neural-Network reconstruction, based on hit positions. Left - Momentum reconstruction for $\mathrm{P}=300,400,500,600 \mathrm{MeV} / \mathrm{c}$ (From top to bottom). Right - Out of plane angle reconstruction for $\phi=0^{\circ}, 8^{\circ}, 16^{\circ}, 24^{\circ}$ (From top to bottom). The standard deviation of the reconstructed momentum $\Delta \mathrm{P} / \mathrm{P}$ is $\sim 7 \%$ for the Momentum range $300-500 \mathrm{MeV} / \mathrm{c}$. The standard deviation of the angular reconstruction $\Delta \phi$ is $\sim 0.5^{\circ}$. 

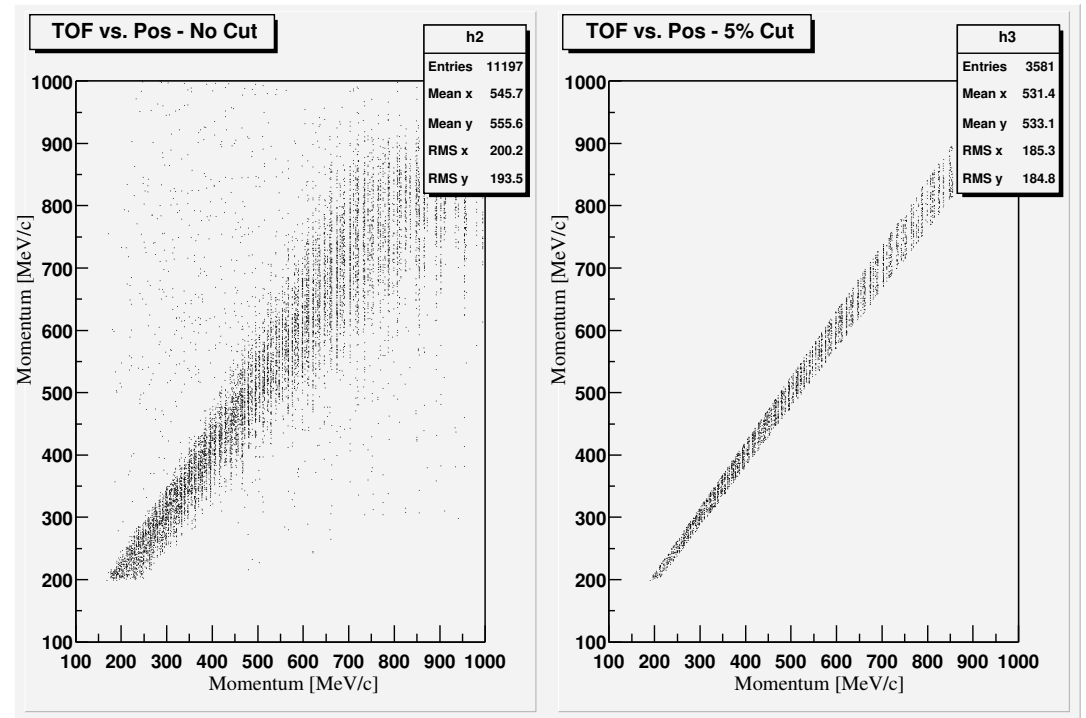

Figure 41: TOF reconstructed momentum before (left) and after (right) position TOF consistency cut. The vertical axis is the momentum reconstructed using TOF. The horizontal axis is the momentum reconstructed using the hit position. ( $50 \%$ background random events were mixed in the simulation).

Energy loss in the $\Delta \mathrm{E}-\mathrm{E}$ scintillators is displayed in figure $42(\mathrm{~B})$. The distributions relating to $\mathrm{k}^{+}$and $\pi^{+}$hits are contained within the dark region at the lower left of the plot. Average $\Delta \mathrm{E} / \mathrm{E}$ energy losses are $0.35 / 5.0 \mathrm{MeV}$ for $\pi^{+}$and $\sim 0.5 / 7.0 \mathrm{MeV}$ for $\mathrm{k}^{+}$. It can be seen that the $\mathrm{E}$ plane stops $\mathrm{d}$ of up to $\sim 80 \mathrm{MeV}$ and $\mathrm{p}$ of up to $\sim 69 \mathrm{MeV}$. Work is in progress to account for finite resolution, non-linear scintillation response at high $\mathrm{dE} / \mathrm{dx}$ and light attenuation in the scintillator bars. Optical photons transport within the plastic is being estimated in a separate Geant4 model, also under development. 

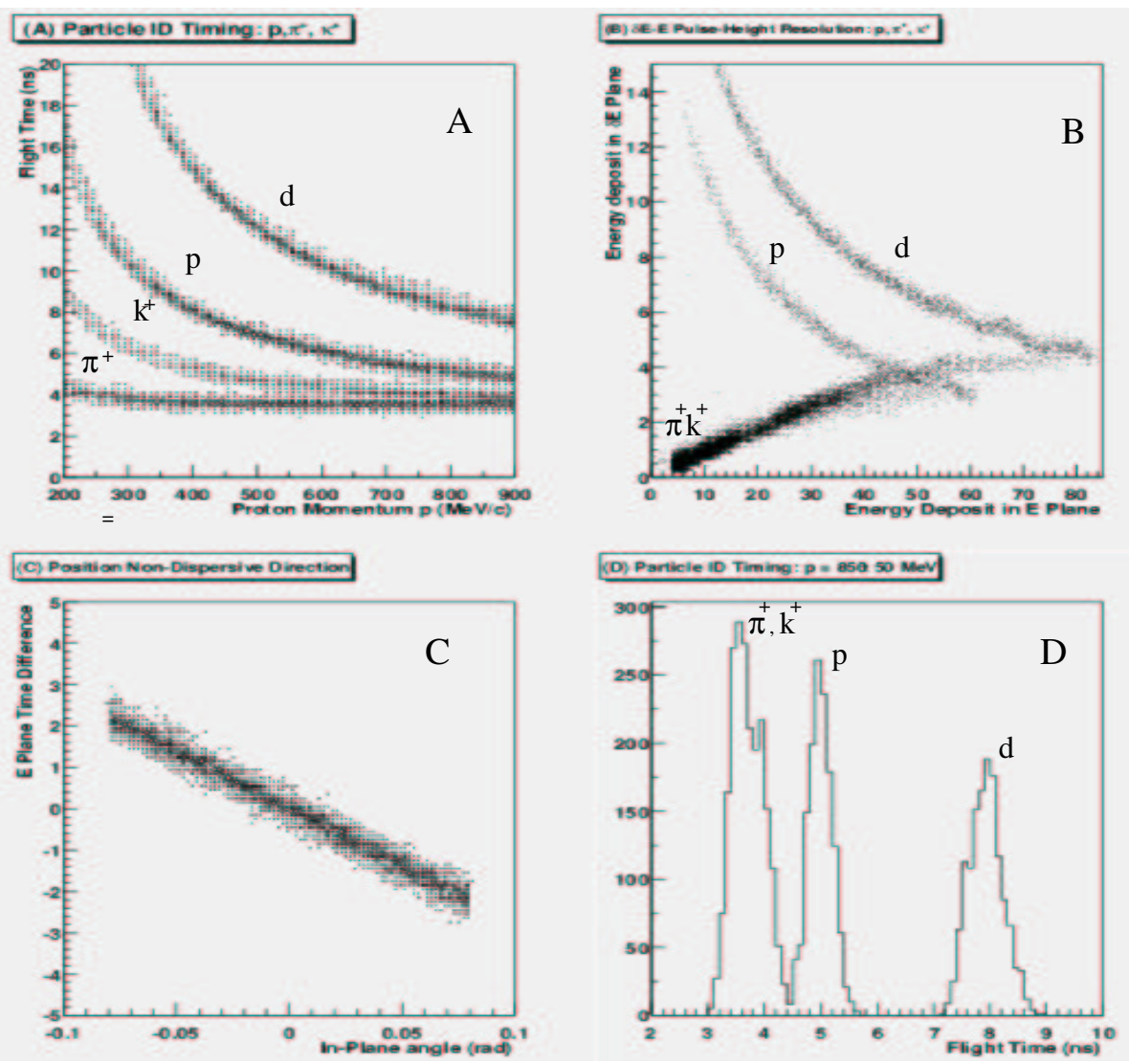

Figure 42: Particle Identification.

A- Flight time from auxiliary to $\Delta \mathrm{E}-\mathrm{E}$ scintillator planes for $\mathrm{d}$ (upper distribution), $\mathrm{p}, \mathrm{k}^{+}$and $\pi^{+}$(lower distribution). B- $\Delta \mathrm{E}-\mathrm{E}$ plots. C- Position info from E plane. D- Flight times for the momentum range $800-900 \mathrm{MeV} / \mathrm{c}$. The right-hand distribution is due to protons while the left results from unresolved $\mathrm{k}^{+}$and $\pi^{+}$. 


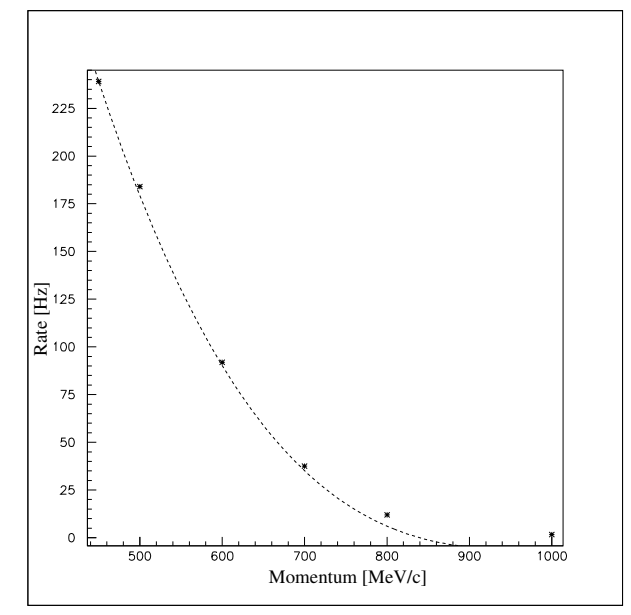

Figure 43: Measured (points) and calculated (dashed line) hit rates in $\mathrm{Hz}$ versus the proton momentum in $\mathrm{MeV} / \mathrm{c}$.

\subsubsection{Singles rates tests and simulations}

A test was conducted in Hall A in order to estimate the singles rate, expected for BigBite during the SRC experiment (E01-15). We have measured the positive particle singles rate for different momentum $(450-1000 \mathrm{GeV} / \mathrm{c})$ using the Hall A left High Resolution Spectrometer (HRS) set at $99.94^{\circ}$. Rates for the SRC Experiment were then estimated based on ratios of momentum acceptance, angular acceptance and luminosity. The estimated rate at the proposed conditions is $3.5 \mathrm{MHz}$. Previous simulation of the same setup predicted a singles positive particle rate of about $8 \mathrm{MHz}$.

The singles low momenta positive particle rate test was done during experiment E01-020 on 11/19/02 (runs 3212 - 3217). The settings which were used for the actual test and for the simulation rates presented in the E01-15 proposal can be seen in Table 2 .

For the HRS rates we used the HRS Left T3 Trigger, as deduced from HALOG and the ESPACE software [18]. We then normalized each of these value to momentum acceptance bins of $1 \mathrm{MeV} / \mathrm{c}$, and to a beam current of $100 \mu \mathrm{A}$ as shown in Table 3 . The normalized rates in Table 3 were obtained by scaling T3 rate by $1 \mathrm{MeV} / \mathrm{c} /$ the HRS momentum acceptance and $100 \mu \mathrm{A} /$ the beam current in $\mu \mathrm{A}$.

To estimate the BigBites singles rate over the entire spectrometer acceptance (250-600 MeV/c) we used the following steps: First, we fit the normalized rates from table 3 using a 3 rd order polynomial fit. (The fit parameters are: A1 = $\left.1.4 \times 10^{3}, \mathrm{~A} 2=-4.18, \mathrm{~A} 3=4.1 \times 10^{-3}, \mathrm{~A} 4=-1.3 \times 10^{-5}\right)$. 


$$
\text { RateFunction }=A_{1}+A_{2} P+A_{3} P^{2}+A_{4} P^{3} .
$$

We then used the fit parameters to integrate over the rate function between the limits 250 and $600 \mathrm{MeV} / \mathrm{c}$, to obtain the rate over the full acceptance -

$$
\text { TotalRate } \left.=\int_{l}^{h} A_{1}+A_{2} P+A_{3} P^{2}+A_{4} P^{3}=A_{1} P+\frac{A_{2} P^{2}}{2}+\frac{A_{3} P^{3}}{3}+{\frac{A_{4}^{4}}{4}}_{p=250}^{p=600}\right]
$$

$\operatorname{Total}(250-600 \mathrm{MeV} / \mathrm{c})=103 \mathrm{KHz}$.

The rate for the $250-600 \mathrm{MeV} / \mathrm{c}$ range per $100 \mu \mathrm{A}$ beam is $=103 \mathrm{KHz}$.

The next step was to calculate the equivalent rate for BigBite, taking in to account the angular acceptance and target differences :-

$$
\begin{aligned}
& A A R=\frac{\text { BB angular acceptance }}{\text { HRS angular acceptance }} \\
& T T R=\frac{\text { Proposedtarget Thickness }}{\text { Testtarget Thickness }} \\
& \text { BigBite Rate }=\text { TotalRate } \times \text { AAR } \times \text { TTR } \\
& \text { BigBite Rate }=3.45 \mathrm{MHz}
\end{aligned}
$$

An additional estimate was done using a Geant simulation of BigBite [19]. First, we used a similar calculation as in the previous page, in order to get the singles rate per $1 \mathrm{msr}, 100 \mu \mathrm{A}$ beam. We integrated over the momentum range of 200 to $1000 \mathrm{MeV} / \mathrm{c}$ which yielded a rate of $48 \mathrm{KHz}$. For the simulation we assumed that the protons are evenly emitted to a total of $174 \mathrm{msr}$ which is bigger than the BigBite acceptance. For this solid angle and momentum distribution, we observed an acceptance efficiency of eff $=0.54$. The singles rate was then calculated as follows :

$$
\begin{aligned}
\text { Rate } & =\text { MeasuredRate }[K H z / m S r] \times \text { TotalSpread }[m S r] \times e f f \\
\text { Rate } & =48[K H z / m S r] \times 174[m S r] \times 0.54=4.5 M H z
\end{aligned}
$$

In summary, the expected singles rates in BigBite in the SRC experiment under the proposal conditions is $4 \pm 0.5 \mathrm{MHz}$. 


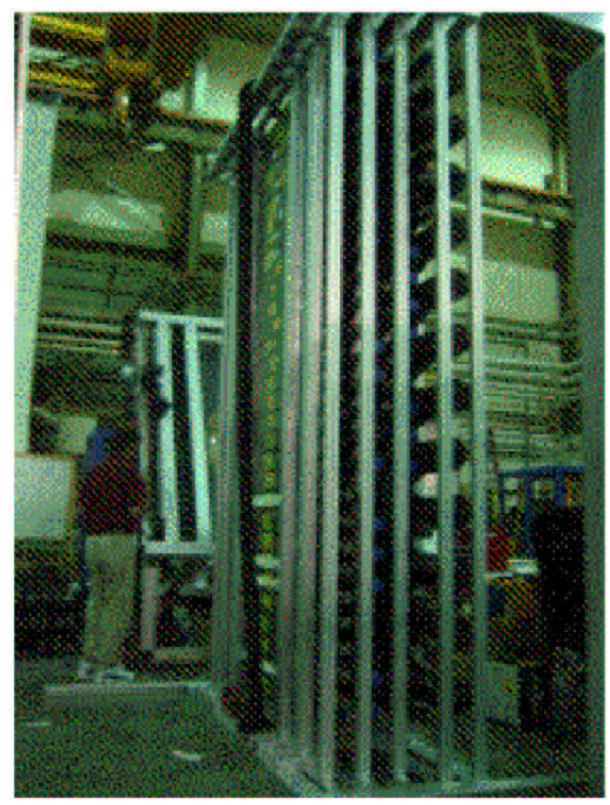

Figure 44: A picture of the neutron array, in the test area at TJNAF.

\section{$3 \quad$ Neutron array for the SRC experiment}

\subsection{Design and building of the neutron-array for the SRC experiment}

The new neutron array for the SRC experiment (see figure 44), consists of four $10 \mathrm{~cm}$ thick segmented scintillator planes and a $2 \mathrm{~cm}$ plane of veto counters in-front of it (not shown in the figure). It is designed to detect neutrons in the momentum range of $200 \sim 600 \mathrm{MeV} / \mathrm{c}$, over the same angular acceptance as BigBite $(\sim 100 \mathrm{msr})$. The neutron array covers an area of $300 \times 100 \mathrm{~cm}^{2}$ and has an effective detection width of $\sim 40 \mathrm{~cm}$. A lead wall will be located in front of the array, shielding the array from background radiation. The direction and size of the incoming neutrons' momentum will be measured using the timing signals from the two PMTs attached to each end of the scintillator bars.

In this chapter we present the array and discuss the cosmic rays tests we did in order to establish its performance.

\subsubsection{The neutron scintillator planes}

The neutron array consists of four scintillator planes for neutron detection (see figure 45). Each of these planes has is $300 \times 100 \times 10 \mathrm{~cm}^{3}$, each having different segmentation. Each of the different bars is attached to a PMT at each of its ends, providing both TOF and hit position along the bars. A schematic view 


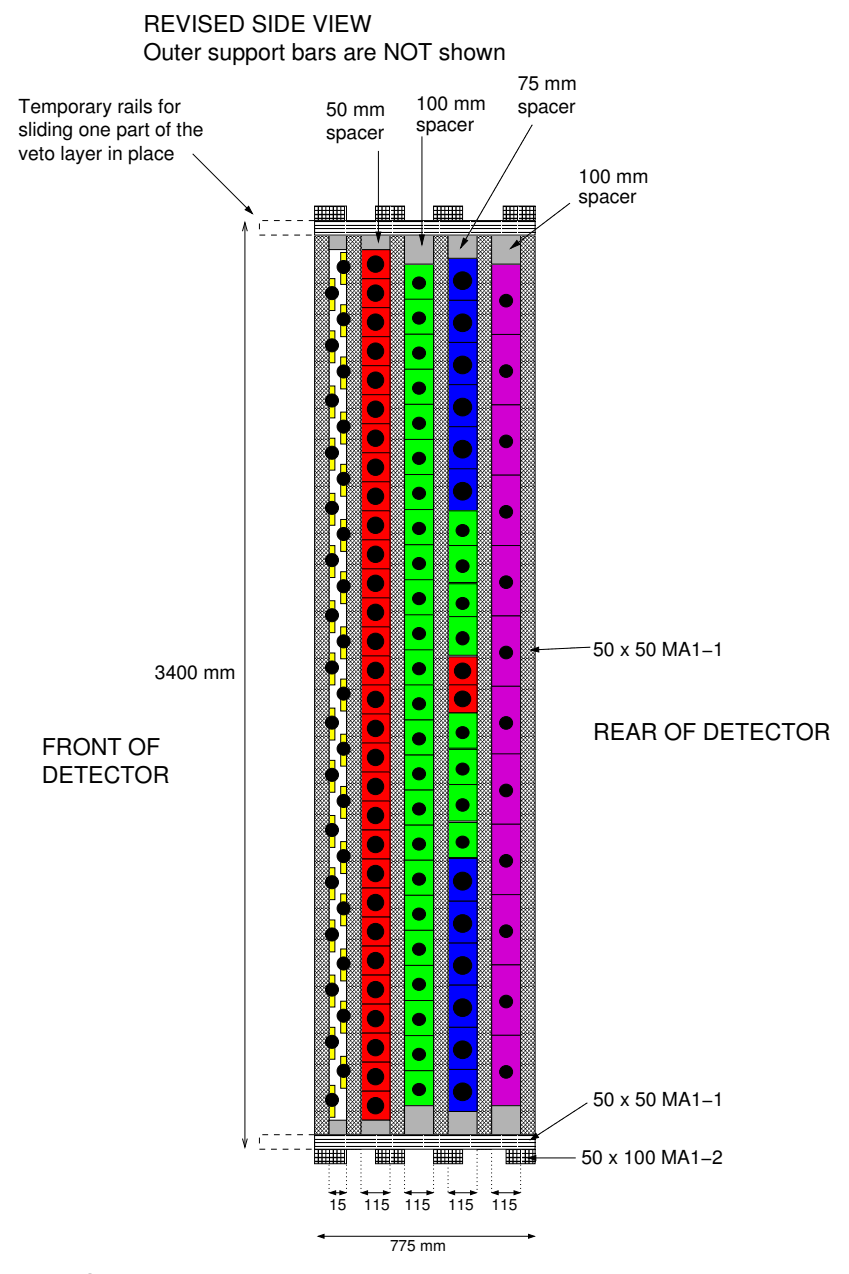

Spacers are the same size at the top and bottom of the planes.

Black dots represent the photomultiplier tubes and bases.

NOTE: The temporary rails (if required) can be detached when the front half of the veto detectors are in place and before the lead wall is put in place.

Figure 45: A schematic side view of the neutron array. The squares are the counters (all 1 meter long, $10 \mathrm{~cm}$ wide but with different heights). The black dots represent the PMTs. There are two PMTs on each side of any of the neutron detectors. 
of the different scintillators used for the array can be seen in figure 46. Each of the scintillator types which were used, are matched to different PMTs. All PMTs were shielded against magnetic field using mu metal covers.

\subsubsection{The Veto layer.}

The front plane of the neutron - array is designed as a "veto layer", to enable detection and separation of charged and neutral particles (see figure 47). A charged particle will create a signal in the Veto scintillators. This signal would be recorded by the DAQ system, and allow us to separate the neutral/charged hits.

\subsection{Tests of Performance}

A complete test with cosmic rays was conducted for this neutron array. The cosmic ray tests were conducted for all neutron counter planes, with all the detector channels connected to the DAQ and using the final complete electronics setup including all delays as planned for the experiment. The electronics for this array are common to the SRC experiment and the $\mathrm{G}_{n}^{E}$ experiment. The electronics setup was assembled by A. Shahinyan. All together 176 ADC channels and 176 TDC channels were tested. These tests where performed as a final check before the detector is moved to Hall A. The tests enabled debugging the counters, PMTs, electronics and DAQ software. The measurements were carried out with the detector package mounted in its support frame and stand as planned for the SRC experiment. Read out was done using CODA and the data was analyzed using ROOT.

\subsubsection{High Voltage setting}

The gain of each of PMT in the neutron array counters was first set using a radioactive ${ }^{228} \mathrm{Th}$ source, which has a Compton peak at $2.26 \mathrm{MeV}$. The detected neutrons in the experiment will have momentum of 250 to $600 \mathrm{MeV} / \mathrm{c}$ and will create recoil charges (typically protons) of lower energies. These recoil protons will have a typical kinetic energy of $100 \mathrm{MeV}$ and a typical range of about $10 \mathrm{~cm}$, roughly the same as the width of the neutron counters. This means that the observed signal would be measured at around $100 \mathrm{MeV}$. The threshold used for the tests was set at about $10 \mathrm{MeV}$-ee, which should result in 8-10\% detection efficiencies of these neutrons. The typical attenuation length of these scintillators is on the order of $1.5 \mathrm{~m}$, which results in a $50 \%$ variation in the measured signal, when placing the source either at the center of the counter, or at the side of the counter. The gain setup was done by placing the source at the center of each counter. Using a x10 amplifier we set the signals from the ${ }^{228} \mathrm{Th}$ source $(2.26 \mathrm{MeV})$ to $40 \mathrm{mV}$. The discriminators were set to $100 \mathrm{mV}$ which is equivalent of a $5.6 \mathrm{MeV}$-ee threshold.

Next we used ADC measurements of cosmic rays to fine-tune the $\mathrm{HV}$ values which were preset using the ${ }^{228} \mathrm{Th}$ source. For each plane, we set up a trigger 


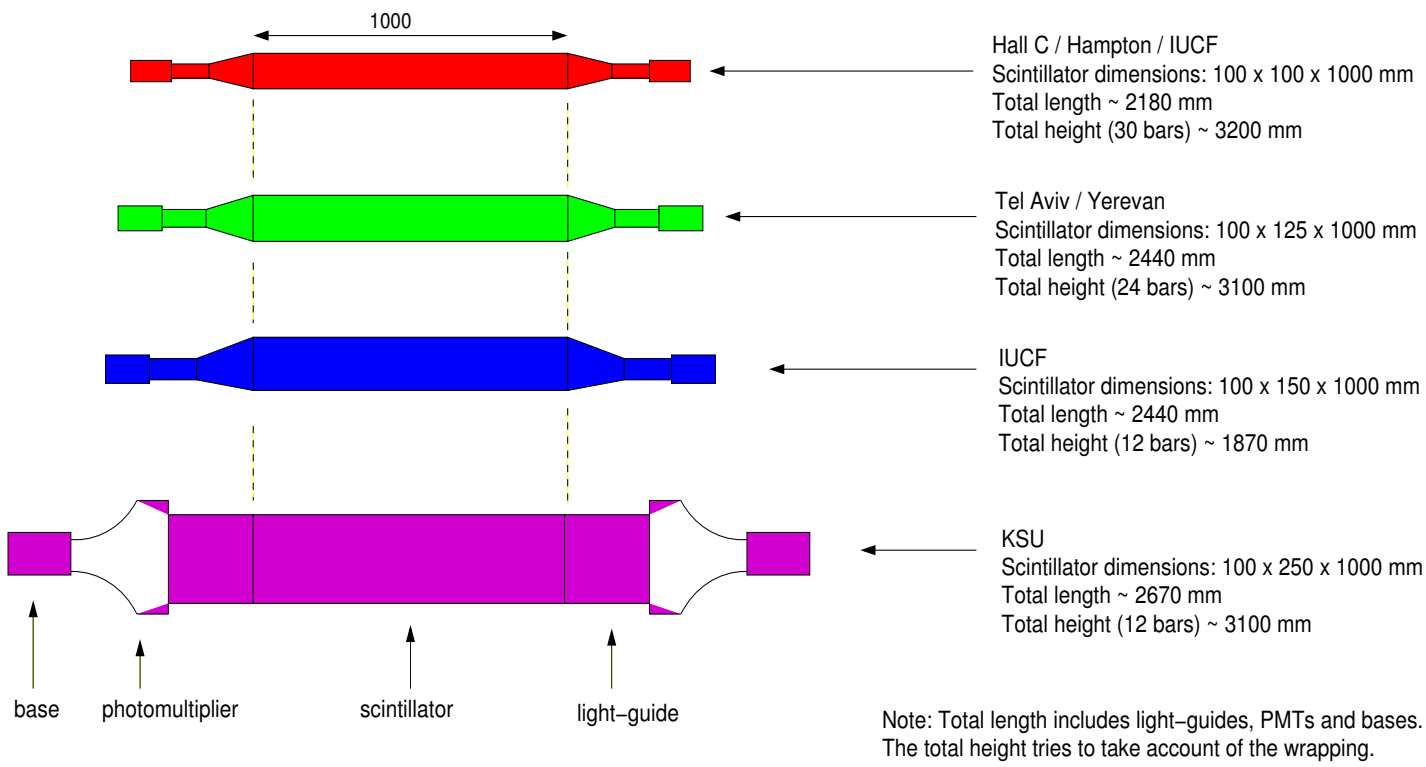

Figure 46: A schematic side view of the different scintillators bars used for the neutron array. 


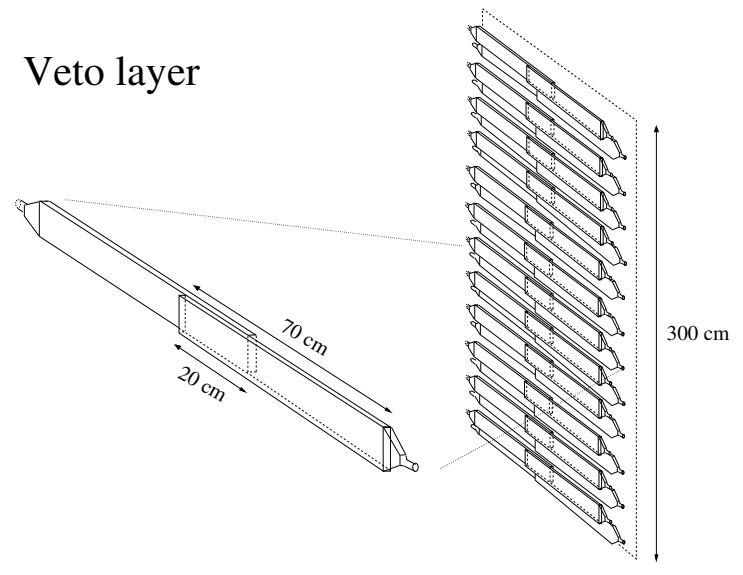

Figure 47: A schematic view of the veto layer in-front of the neutron array.

of Top counter AND Bottom counter, allowing only vertical rays which crossed both counters (see figure 49-A). The HV was adjusted for each counter, so a 20 MeV-ee energy deposit (sharp edge of energy deposit spectra) will correspond to channel 1600 (which is about $20 \%$ of the full scale). See figure 50 for a typical ADC spectra for these cosmic rays and counters of width 10, 12 and $25 \mathrm{~cm}$.

\subsubsection{Position calibration}

Position along each of the counters in the array was calibrated using Cosmic rays (see figure 51 ). The position along the bar is given by -

$$
x=A\left(\mathrm{~T}_{r}-T_{l}\right)+\mathrm{B} .
$$

Tr is the right PMT time, Tl is the left PMT time and A, B are the calibration parameters. A and B were found for each counter by fitting the $\mathrm{x}$ distribution to a step-function centered at zero and with the same length as the physical counter length $(1 \mathrm{~m})$. The trigger used for this measurement was an OR of all the counters.

A check of that calibration and a measurement of the position resolution along the counters was then performed using a fiber scintillator $(2 \mathrm{~cm}$ in width), which was placed perpendicular to the array on the front plane (see figure 49B). The trigger was set to allow only center positioned rays which trigger both the fiber AND one of the counters in the array. This resulted in a narrow centered position distribution (see figure 51 bottom) with a width of $\sigma=7.6 \mathrm{~cm}$. This position resolution is given by the time resolution of each PMT multiplied by $\sqrt{2}$ multiplied by the speed of light in the scintillator. $\sigma=\sqrt{2} \times 0.2 \mathrm{~ns} \times 20 \mathrm{~cm} / \mathrm{ns} \sim 7 \mathrm{~cm}$. 


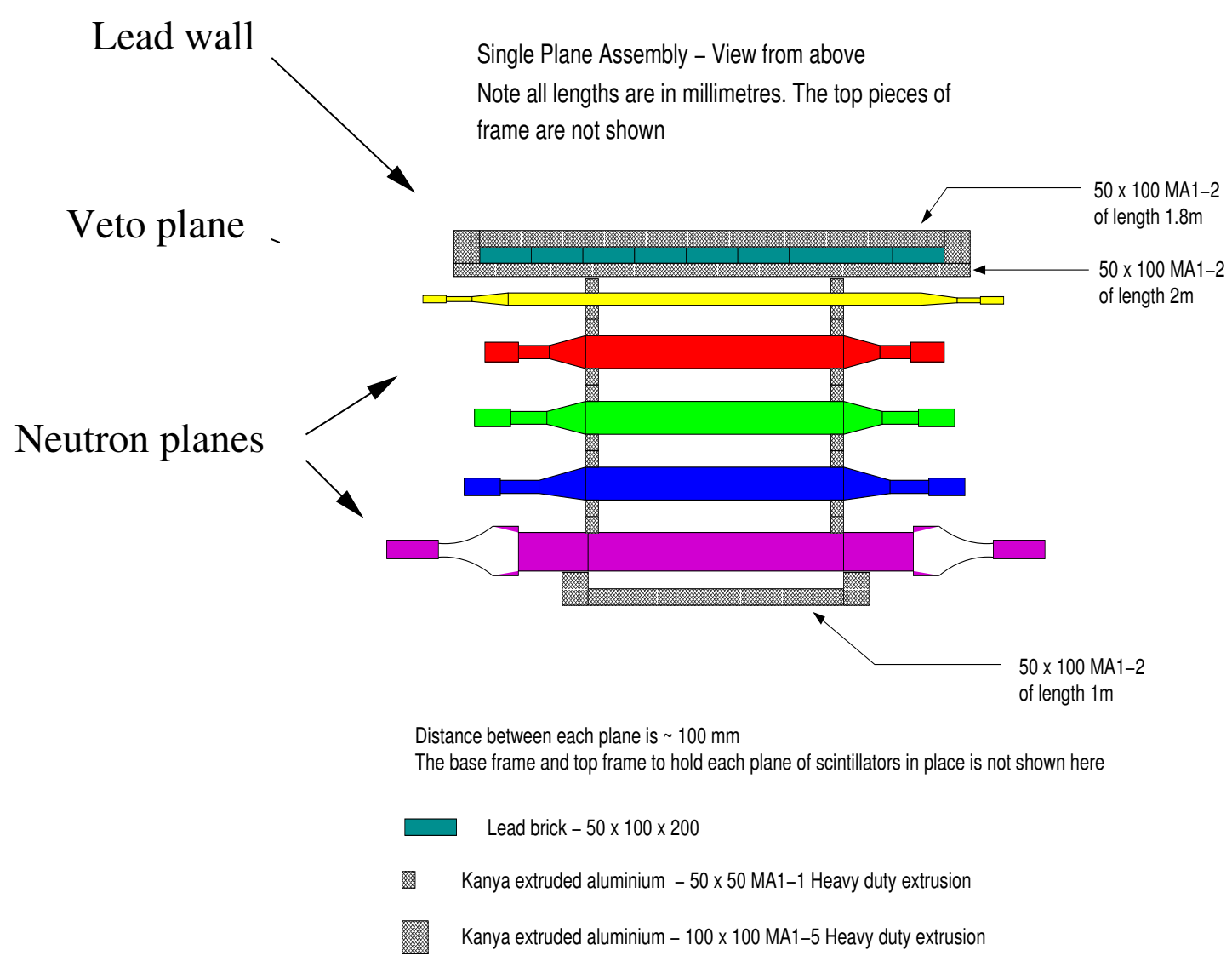

Figure 48: Top view of the neutron array.

From top to bottom - The front lead wall, the veto layer and the four neutron detection planes. 


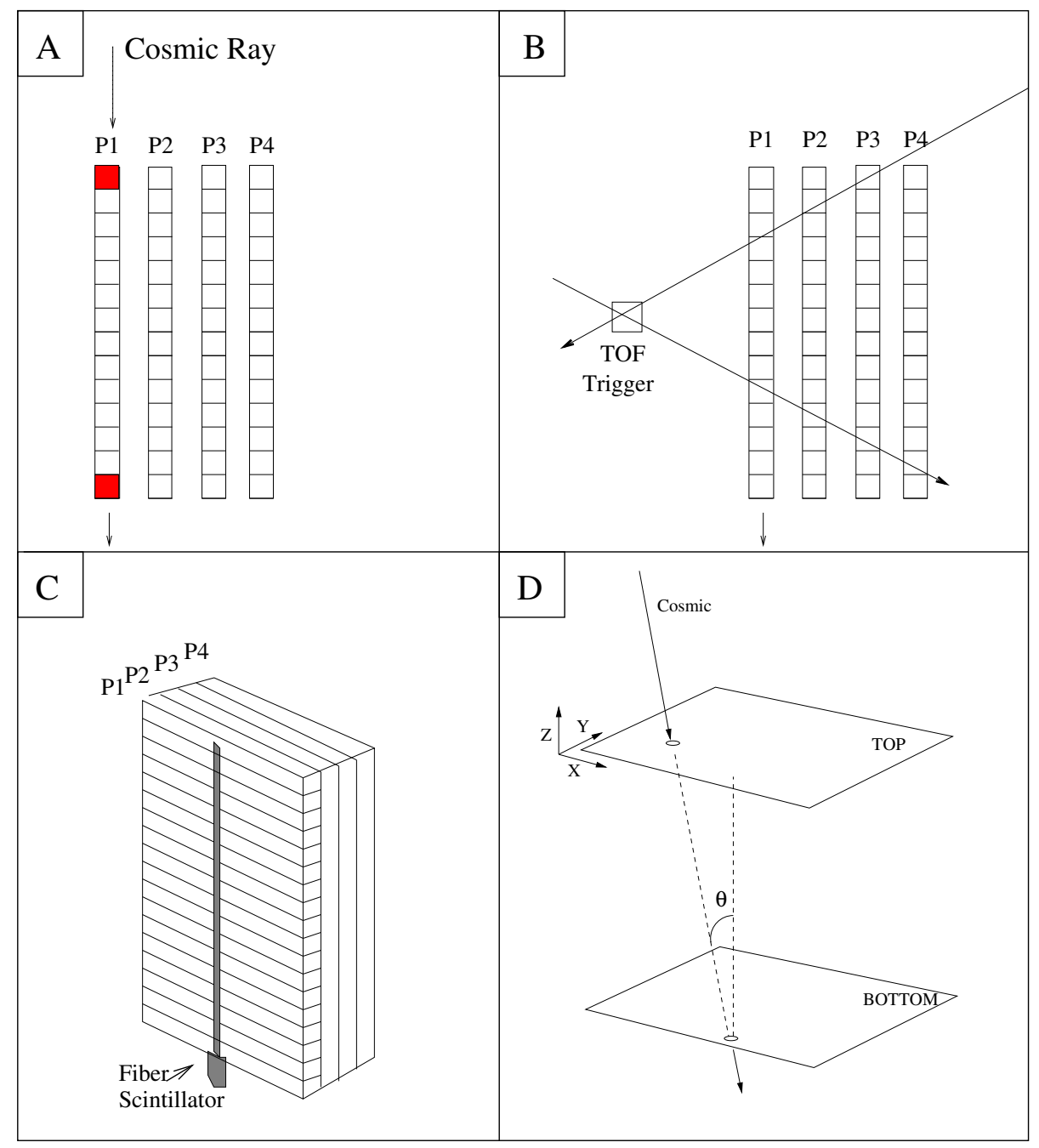

Figure 49: Different experimental setups for the cosmic rays tests. 

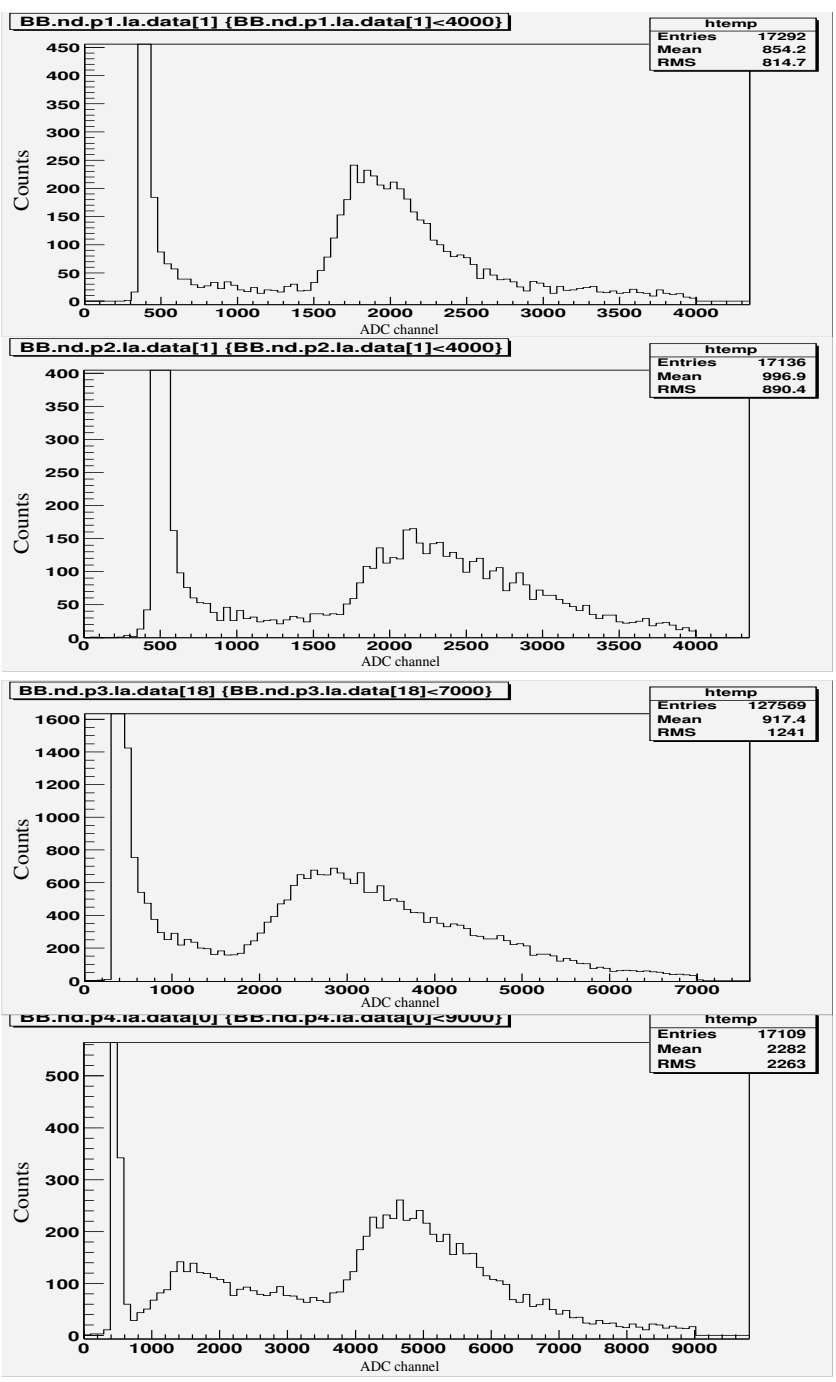

Figure 50: Typical ADC spectrum for cosmic ray going across the counter as shown in figure $49 \mathrm{~A}$. Top to Bottom are typical spectra for a $10 \mathrm{~cm}, 12.5 \mathrm{~cm}$, $15 \mathrm{~cm}$ and $25 \mathrm{~cm}$ thick counters. 


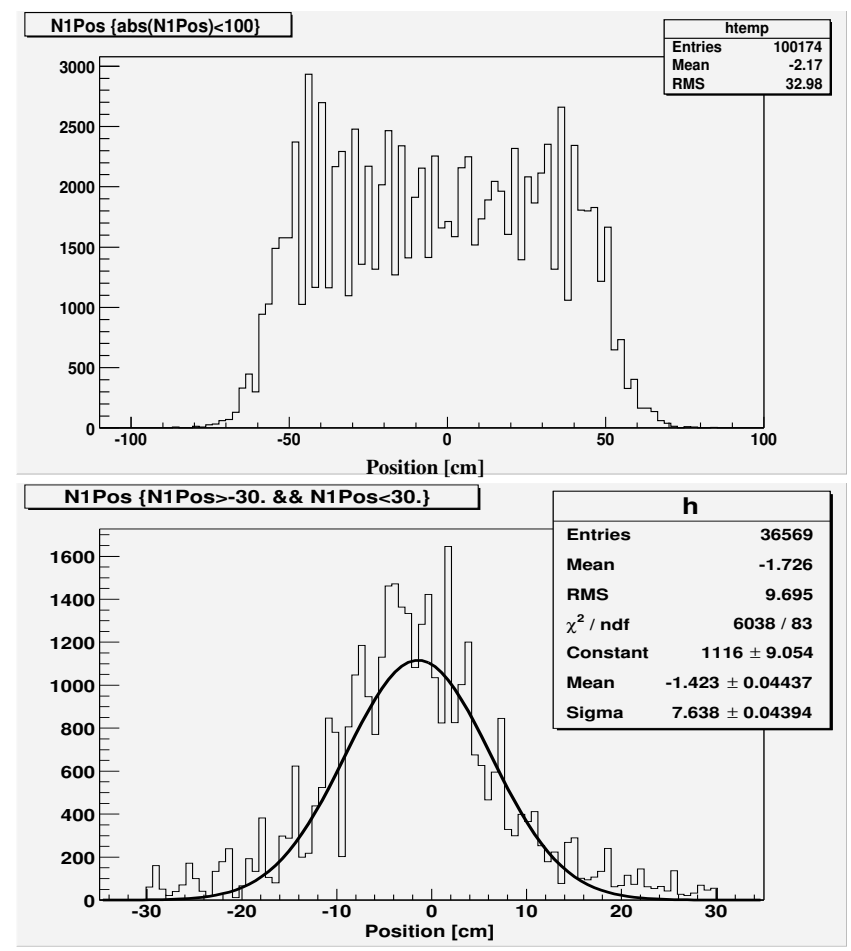

Figure 51: Measured position along the entire front plan.

Top - with no fiber optic trigger. Bottom - using a coincidence trigger with a fiber scintillator $(\sigma=7.6 \mathrm{~cm})$.

The position resolution for the BigBite E plane counters was measured to be $5 \mathrm{~cm}$, which is slightly better. The measured position resolution corresponds to an horizontal angular resolution of $\sigma \sim 15 \mathrm{mrad}$ in the planned position of the detector during the SRC experiment (5 meters away from the target).

\subsubsection{TOF calibration}

The TOF for each counter was calibrated using the known TOF/meter for cosmic rays (assumed to be at the speed of light). First, we used a 10x10x100 cm counter located at distance of $340 \mathrm{~cm}$ from the array and required a coincidence of it and any of the counters in the array. (see figure 49B). The measured TOF distribution contains two peaks, positioned $6.6 \mathrm{~ns}$ apart, which correlates to forward and backwards going cosmic rays (see figure 53 top). After determining the timing resolution for each counter (see figure 52), we fixed all the counters to one absolute time. The TOF/meter for each counter was fitted by adjusting the A, B parameters with -

$$
\text { TOF } / \text { meter }=A \frac{(T l+T r) / 2-(T l(\text { trigger })+T r(\text { trigger })) / 2}{\text { Distance }}+C,
$$


where $\mathrm{Tl}$ and $\mathrm{Tr}$ are the left and right PMTs times, $\mathrm{Tl}$ (trigger) and $\operatorname{Tr}$ (trigger) are the trigger times and Distance is the physical distance between the hit position on the trigger counter and the hit position along the counter in the array.

$$
\left(\text { Distance }=\sqrt{\Delta X^{2}+\Delta Y^{2}+\Delta Z^{2}}\right) .
$$

$\mathrm{C}$ is the calibrated parameter which was found by centering all the distributions at $3.3 \mathrm{~ns} /$ meter. The measured TOF/meter for a single typical counter in the first plane can be seen in figure 53-bottom. The obtained resolution $(\sigma)$ for all counters in the four planes are shown in figure 52 .

\subsubsection{Attenuation length studies}

In order to study the attenuation length in the scintillator counters (see section 1.1), we plot the ADC signal versus the position along the bar. We used the same trigger as used for the ADC calibration (see figure $49 \mathrm{~A}$ ) in order to get signals only from cosmic rays which crossed the bars in a straight path from top to bottom. We applied an additional cut by demanding $A D C_{l e f t} \times \mathrm{ADC}_{\text {right }}$ to be within a specific region. This multiplication of the signal from the two ends of the scintillator was found to be almost position independent. The attenuation length was then found by fitting the ADC versus position distribution to an exponential function (see figure 54). The measured attenuation length was between 100 to $200 \mathrm{~cm}$, depending on the specific counter.

\subsubsection{Summary and conclusions}

The tests indicate that TOF resolution better than $\sigma=0.3 \mathrm{~ns}$ can be achieved with the array. In the planned position for the array during the SRC experiment (5 $\mathrm{m}$ away from the target), this will result in a momentum resolution for the recoil neutron momentum of the order $\Delta \mathrm{P} / \mathrm{P}=\Delta \mathrm{TOF} / \mathrm{TOF}=0.3 / 30=1 \%$, which is better then the momentum resolution for the recoil protons detected by BigBite $(\Delta \mathrm{P} / \mathrm{P}=3 \%)$.

Position measurements along each of the bars will have typical resolution resulting from the time resolution of each PMT and the speed of light in the scintillator.

$\sigma=0.3 \mathrm{~ns} \times 20 \mathrm{~cm} / \mathrm{ns} \sim 7 \mathrm{~cm}$.

This resolution in position corresponds to a horizontal angular resolution of $\sigma \sim 15 \mathrm{mrad}$ during the experiment. At the point this report is written, there are 5 bars with PMT or electronic problems to be fixed (see fig 52). These will be fixed and tested before we move the array to the hall for the experiment.

\subsection{The neutron array singles rates and shielding test}

Three $50 \mathrm{~cm}$ neutron bars were set-up on a test platform in Hall A at the Jefferson Lab, each with differing amounts of radiation shielding in order to 


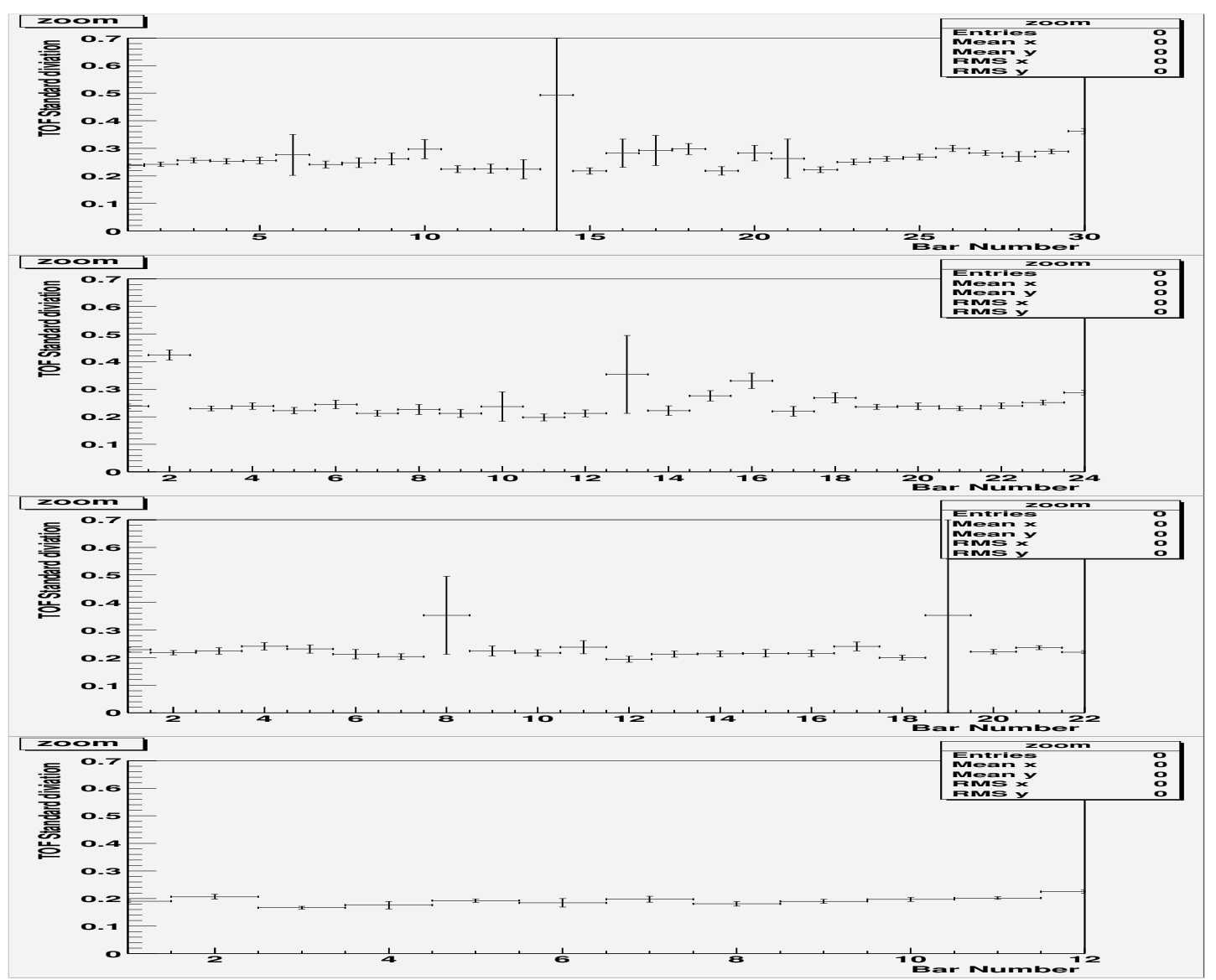

Figure 52: Timing resolution ( $\sigma$ ) for each counter in the array.

Counters at planes P1, P2, P3 ,P4 are shown from Top to bottom. P1 is the front plane and $\mathrm{P} 4$ the back plane. Counter 1 is the top counter in each plane. There are 30,24,22,12 counters in planes $\mathrm{P} 1$ to $\mathrm{P} 4$ respectively. 


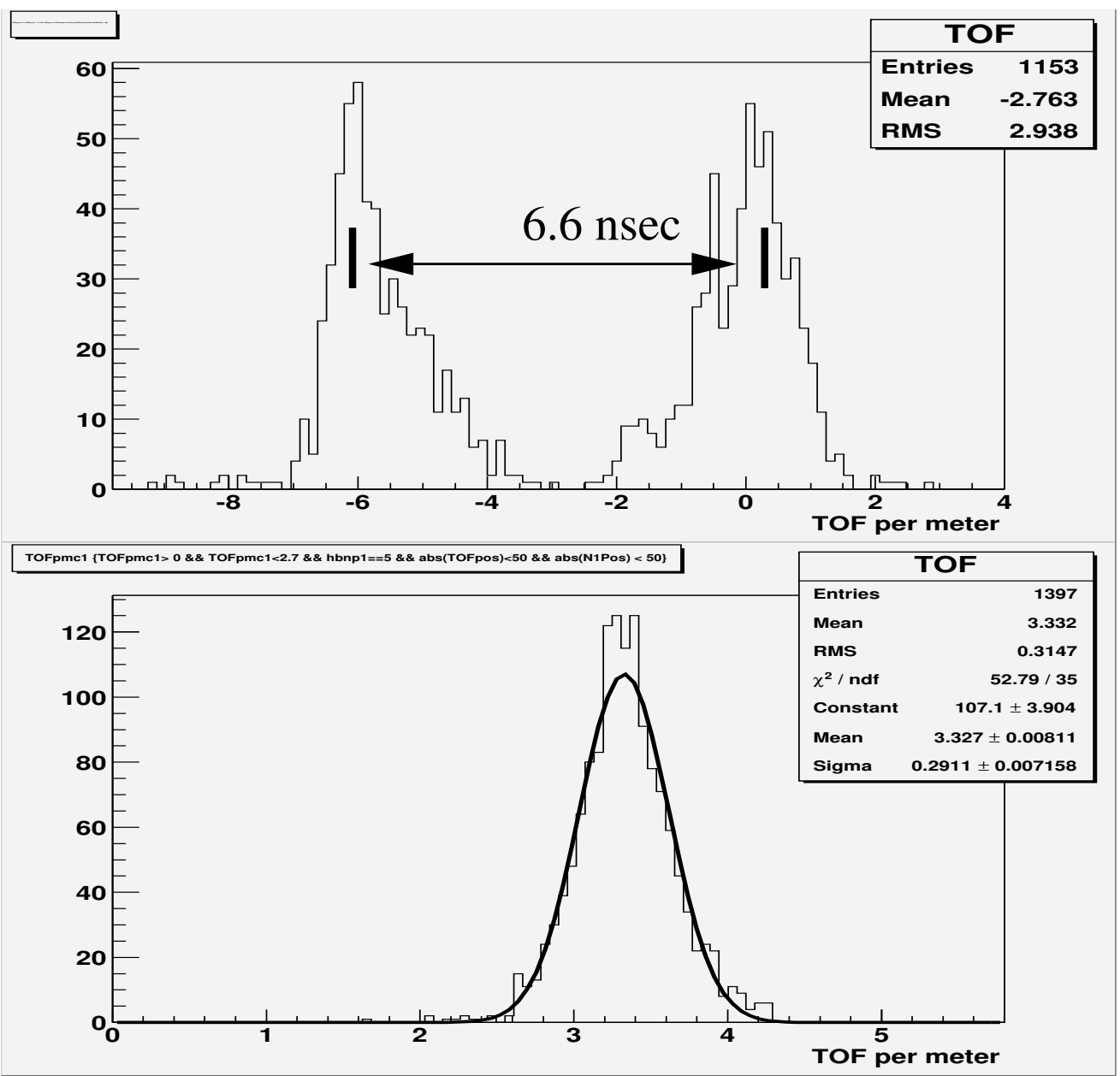

Figure 53: Top: TOF/m for a typical P1 counter - before the shift and calibrations. Bottom - a single counter peak after shift.

The TOF $/ \mathrm{m}$ resolution in this case is $\sigma \sim 290 / \sqrt{2}=200 \mathrm{ps}$. 


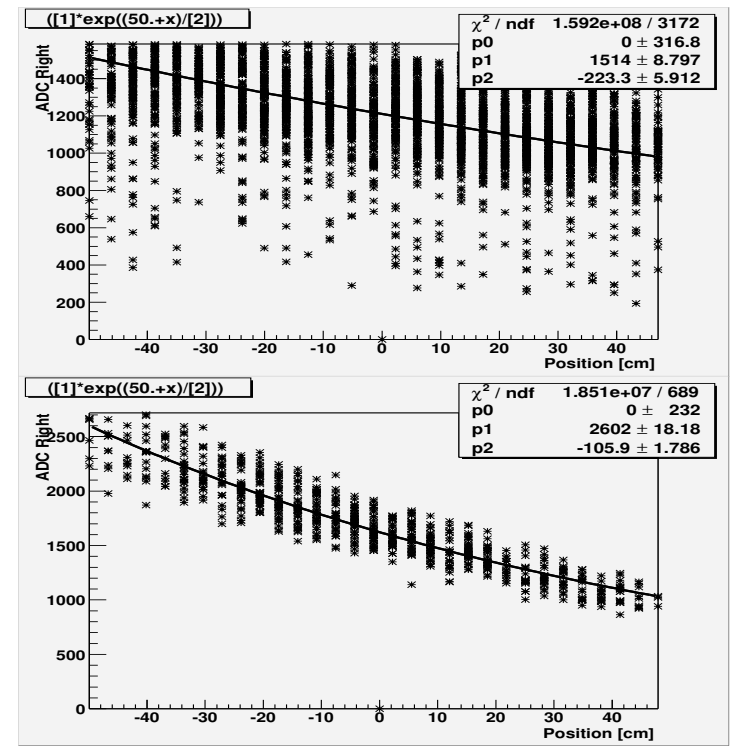

Figure 54: ADC (right) versus position along the counters.

Top panel shows a plane 1 counter with $\mathrm{L}=223 \mathrm{~cm}$. Bottom - a plane 2 counter with $\mathrm{L}=105 \mathrm{~cm}$.

determine the most efficient use of shielding (see figure 55). By counting the number of coincident signals from the two photo multiplier tubes attached to either end of each bar, we try to determine the effect of the room background radiation and the best shielding configuration. The results indicate that the room background is only a small percentage of the detected radiation compared to the amount of radiation coming from the target [20]. The results also show that only a front lead shield will be required for the proposed neutron detector for the SRC experiment. To test the difference between various amounts of radiation shielding, the three neutron bars were placed on a test platform in the hall. This platform was around 4-5 feet below beam height, at an angle of $125^{\circ}$ and 7 meters from the target. A small concrete wall, 24 inches high and 18 inches deep, was built so that two of the bars could be nearer to beam height (beam height in the hall is 10 feet). Bar \#2 was placed on top of the concrete wall and a lead wall built around it. A cover of $1 \times 6 \times 12$ inch lead bricks was placed on the wall surrounding bar \#2. Then bar \#1 was placed on top of this lead case and a wall of lead bricks (2 inches thick) was placed in front of it. Bar \#3 was placed on a wooden block on the test platform, behind the concrete wall. We did not have enough concrete blocks to build a shield 4 feet thick so instead we put 2.5 feet of concrete followed by a 2 inch thick wall of lead bricks to simulate approximately 4 feet of concrete. Thus, bar \#3 will test room background radiation and not the radiation coming from the target. The voltage supply for the neutron bars was calibrated using both a ${ }^{228} \mathrm{Th}$ source, 


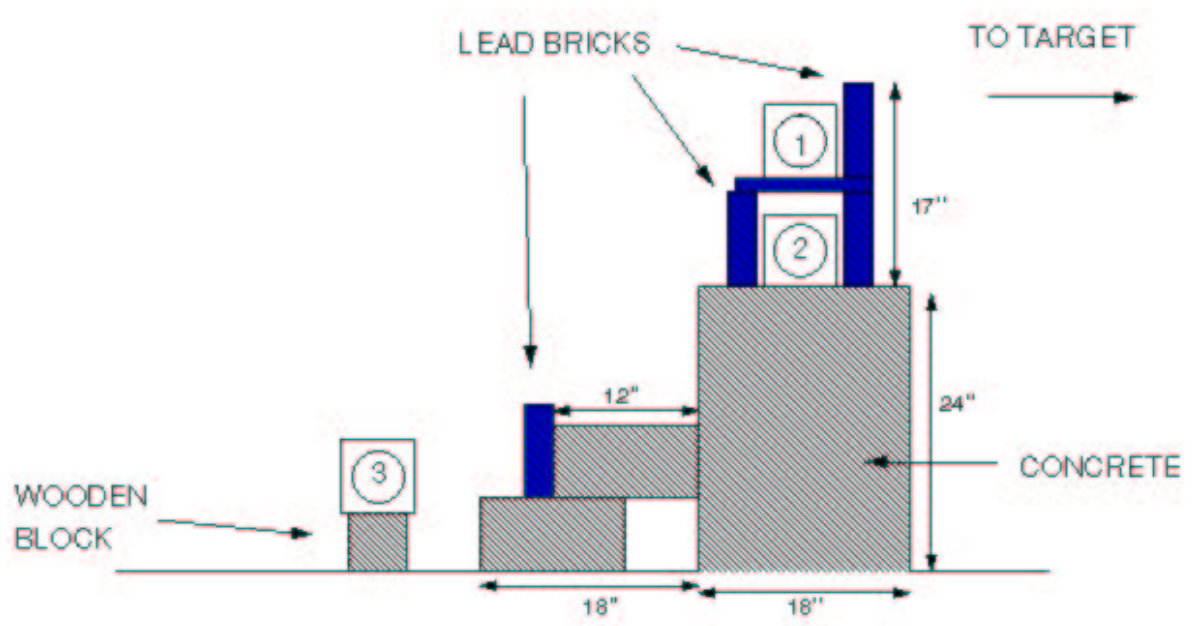

Detectors labelled 1,2 and 3

Figure 55: The different shielding configurations used for the shielding test. The beam height is about 2 feet above bar \# 1 .

and cosmic rays (see table 4). To obtain each set of results, the experiment was run for approximately 30 minutes and the the number of counts in each bar was noted. Data was taken at several different discriminator threshold settings (see figure 56). The target used during the experiment was a liquid deuterium target and the beam current was over $100 \mu \mathrm{A}$.

The results of each test are given in table 5. The number of counts for each bar indicate that in every case, bar \#3 counts significantly less then either of the other two bars. This means that the room background radiation is much less than the radiation from the target. There is also very little difference between the number of counts registered by bar \#1 and \#2 which means that little is gained by completely enclosing the neutron bar in a lead shield. Hence, this means that a single lead shield in front of the neutron detector should provide sufficient shielding, and that a detection threshold of 10-15 MeV-ee will provide a high enough detection efficiency relative to the singles rates. 


\section{Ratio of room to target radiation as a function of threshold}

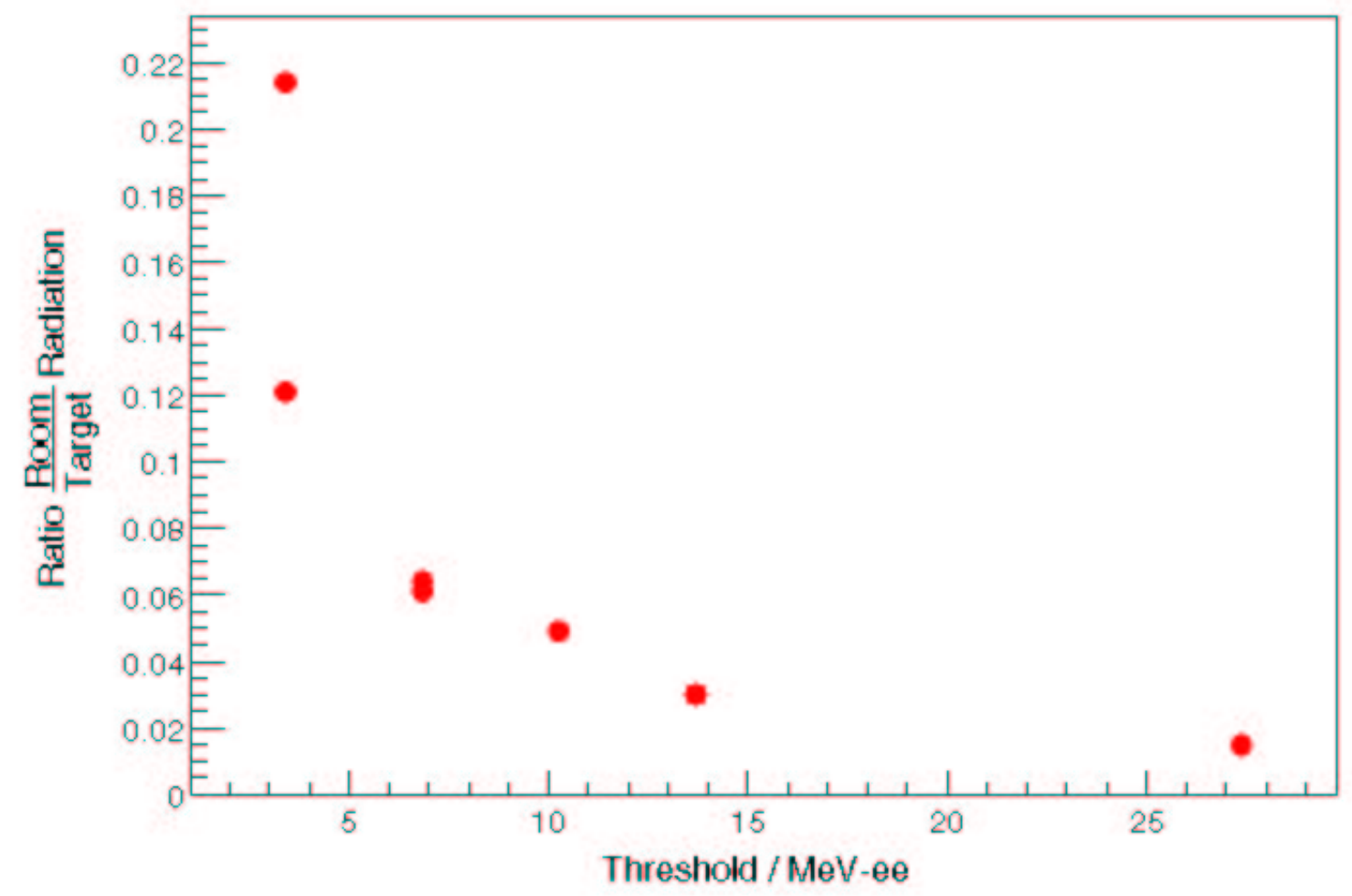

Figure 56: The ratio of the number of counts from bar \#3 (room) to bar \#1 (target) as a function of threshold energy of the incoming neutron.

Note, that two of the points at the lowest threshold were duplicated from the two tests at a different time from the rest of the test. 


\section{References}

[1] E.J Brash, R.D. Ransome, and C.W. de Jager, Status Report on Activities in Hall A, 1999 (unpublished).

[2] E. Piasetzky, W. Bertozzi, J. Watson, S. Wood (spokespersons). Studying the internal small-distance structure of nuclei via the triple coincidence (e,e'pN) measurement. Experiment 97-106 Proposal to Jefferson Laboratory, 1997 (unpublished).

[3] Progress Report on the Auxiliary Plane, for the Triple Coincidence Experiment with BigBite, 2001, (unpublished).

[4] BigBite Trigger Specification and Costing, 2001, (unpublished). http://hallaweb.jlab.org/ annand/BBcosts.ps

[5] D. Johnston and E. Piasetzky, Scattering Off a Moving Pair in Nuclei (unpublished). http://hallaweb.jlab.org/equipment/BigBite/e01015-mc.ps.

[6] B. Reitz, B. Wojtsekhowski, K. McCormick, G, Cates, Measurement of the Neutron Electric Form Factor $G_{n}^{E}$ at High Q2, TJNAF Hall A proposal E02-013, 2001, (unpublished).

[7] W. Bertozzi, Z.L.Zhou, D.W.Higinbotham, S.Sirca, B.E.Norum, Measurement of $\mathrm{Ax}$ and $\mathrm{Az}$ asymmetries in the quasi-elastic pol $3 \mathrm{He}(\mathrm{pol}$ e,e'd) reaction, TJNAF Hall A proposal E02-108, 2002, (unpublished).

[8] B.E.Norum, K.Wang, T.Tamae, W. Bertozzi, Exclusive Study of Deuteron Electrodisintegration Near Threshold, TJNAF Hall A proposal E02-101, 2002, (unpublished).

[9] R.Lindgren, D.W.Higinbotham, J.R.M Annand, V. Nelyubin, Precision Measurement of Electroproduction of pi0 near Threshold: A Test of Chiral QCD Dynamics, TJNAF Hall A proposal E01-014, 2003, (unpublished).

[10] R. Fernow et al., Introduction to experimental particle physics 1986.

[11] Philips Photonics, Photomultiplier tubes, Principles and applications 1994.

[12] J.R.M Annand and D.Hamilton et al., Geant4/C - Monte Carlo Studies of the BigBite Focal-Plane Detector System 2001 (unpublished).

[13] Time resolution determination for BigBite trigger plane and auxiliary plane, 2002, (unpublished).

http://hallaweb.jlab.org/equipment/BigBite/Reports/aux-plane-tests.ps

[14] The Jefferson lab data acquisition group, CODA - CEBAF On-Line Data Acquisition User's Manual, 1999 (unpublished).

[15] R.Brun (CERN), ROOT An object-Oriented Data Analysis Framwork, User Guide 2003 (unpublished). 
[16] O.Couet (CERN), PAW Physics Analysis Workstation Tutorial, 1999 (unpublished).

[17] P.E. Ulmer MCEEP - Monte Carlo for Electro-Nuclear Coincidence Experiments, 2001, (unpublished).

[18] ESPACE manual version 2.9.0, Event Scaning Program for Hall A Collaboration Experiments, The Hall A Collaboration 2002, (unpublished).

[19] R. Madey et al., Nucl. Inst. and Meth. 214, (1983) 401.

[20] High Luminosity Operation of a Large Solid Angle Neutron Detector Array in Jefferson Lab's Hall A 2001 (unpublished). 
Table 1: The momentum reconstruction resolution, using simulated TOF between auxiliary plane and the trigger plane, and between the target and the trigger plane.

\begin{tabular}{|c|c|c|}
\hline $\mathrm{P}[\mathrm{MeV}]$ & $\Delta \mathrm{P} / \mathrm{P}$ Aux-E $\%$ & $\Delta \mathrm{P} / \mathrm{P}$ Target-E $\%$ \\
\hline 300 & 6 & 3 \\
400 & 9 & 3.5 \\
500 & 14 & 6 \\
\hline
\end{tabular}

Table 2: Test versus Simulation parameters.

\begin{tabular}{|c|c|c|}
\hline Parameter & Test with HRS & BB Simulation \\
\hline Beam Energy $[\mathrm{GeV}]$ & 5.006 & 4.00 \\
Beam Current $[\mu \mathrm{A}]$ & $4-50$ & 100 \\
Detector Angle $[\mathrm{deg}]$ & 99.94 & 90.0 \\
P Momentum $[\mathrm{MeV} / \mathrm{c}]$ & $450-1000$ & 400 \\
P Acceptance $[\mathrm{MeV} / \mathrm{c}]$ & $.09 \% \times \mathrm{P}$ & $250-600$ \\
Target & Carbon & Carbon \\
Target Thickness $[\mathrm{mm}]$ & 0.5 & 1 \\
Target Angle $[\mathrm{deg}]$ & 90 & 50 \\
Dead Time & $5 \%$ & $0 \%$ \\
\hline
\end{tabular}

Table 3: Measured and calculated rates.

\begin{tabular}{|c|c|c|c|c|}
\hline Run & $\mathrm{P}[\mathrm{MeV}]$ & Current $[\mu \mathrm{A}]$ & T3 Rate $[\mathrm{Hz}]$ & Rate $[\mathrm{Hz}]$ per $1[\mathrm{MeV} / \mathrm{c}]$ and $100[\mu \mathrm{A}]$ \\
\hline 3212 & 1000 & 42.68 & 63.5 & 1.65 \\
3213 & 800 & 50.91 & 439.4 & 11.98 \\
3214 & 700 & 4.39 & 103.9 & 37.56 \\
3215 & 600 & 4.19 & 207.9 & 91.88 \\
3216 & 500 & 4.50 & 372.7 & 184.04 \\
3217 & 450 & 3.93 & 380.6 & 239.10 \\
\hline
\end{tabular}


Table 4: The number of counts from each bar with cosmic rays.

\begin{tabular}{|c|c|c|c|}
\hline Bar \# & Threshold $[\mathrm{mV}]$ & Threshold $[\mathrm{MeV}]$ & Counts \\
\hline 1 & & & 2913 \\
2 & -30 & 1.71 & 4816 \\
3 & & & 2698 \\
\hline 1 & & & 2362 \\
2 & -60 & 3.42 & 2362 \\
3 & & & 2341 \\
\hline 1 & & & 1757 \\
2 & -120 & 6.84 & 1792 \\
3 & & & 1757 \\
\hline
\end{tabular}

Table 5: The parameters and results from the shielding test.

The ratio of $\frac{\text { bottom }}{\text { top }}$ refers to the ratio of counts from bar \#3 (room) and bar

\begin{tabular}{|c|c|c|c|c|c|c|}
\hline Bar \# & Duration & Threshold [mV] & Threshold [MeV-ee] & Current & counts & $\frac{\text { bottom }}{\text { top }}$ \\
\hline $\begin{array}{l}1 \\
2 \\
3\end{array}$ & 25 mins & $-60 \mathrm{mV}$ & 3.42 & $109 \mu \mathrm{A}$ & $\begin{array}{l}866060502 \\
711158224 \\
182529699\end{array}$ & 0.214 \\
\hline $\begin{array}{l}1 \\
2 \\
3\end{array}$ & $24 \mathrm{mins}$ & $-60 \mathrm{mV}$ & 3.42 & $110 \mu \mathrm{A}$ & $\begin{array}{c}662809913 \\
733382234 \\
80508176\end{array}$ & 0.121 \\
\hline $\begin{array}{l}1 \\
2 \\
3\end{array}$ & 28 mins & $-120 \mathrm{mV}$ & 6.84 & $109 \mu \mathrm{A}$ & $\begin{array}{c}340265395 \\
346901725 \\
21735303\end{array}$ & 0.064 \\
\hline $\begin{array}{l}1 \\
2 \\
3\end{array}$ & 26 mins & $-120 \mathrm{mV}$ & 6.84 & $110 \mu \mathrm{A}$ & $\begin{array}{c}334153059 \\
330163520 \\
20494541\end{array}$ & 0.061 \\
\hline $\begin{array}{l}1 \\
2 \\
3\end{array}$ & 26 mins & $-180 \mathrm{mV}$ & 10.26 & $109 \mu \mathrm{A}$ & $\begin{array}{c}235662810 \\
236009916 \\
11455693\end{array}$ & 0.049 \\
\hline $\begin{array}{l}1 \\
2 \\
3\end{array}$ & 26 mins & $-240 \mathrm{mV}$ & 13.68 & $110 \mu \mathrm{A}$ & $\begin{array}{c}183005721 \\
177960767 \\
5569794\end{array}$ & 0.03 \\
\hline $\begin{array}{l}1 \\
2 \\
3\end{array}$ & 26 mins & $-480 \mathrm{mV}$ & 27.36 & $109 \mu \mathrm{A}$ & $\begin{array}{c}42748383 \\
33083387 \\
637698\end{array}$ & 0.015 \\
\hline
\end{tabular}

